University of Tennessee Health Science Center

UTHSC Digital Commons

$12-2014$

\title{
Effect of Nedd4 Haploinsufficiency on Insulin Sensitivity, Adiposity and Neuronal Behaviors
}

Jingjing Li

University of Tennessee Health Science Center

Follow this and additional works at: https://dc.uthsc.edu/dissertations

Part of the Endocrine System Diseases Commons, Genetic Processes Commons, and the Medical Genetics Commons

\section{Recommended Citation}

Li, Jingjing , "Effect of Nedd4 Haploinsufficiency on Insulin Sensitivity, Adiposity and Neuronal Behaviors" (2014). Theses and Dissertations (ETD). Paper 141. http://dx.doi.org/10.21007/etd.cghs.2014.0181.

This Dissertation is brought to you for free and open access by the College of Graduate Health Sciences at UTHSC Digital Commons. It has been accepted for inclusion in Theses and Dissertations (ETD) by an authorized administrator of UTHSC Digital Commons. For more information, please contact jwelch30@uthsc.edu. 


\title{
Effect of Nedd4 Haploinsufficiency on Insulin Sensitivity, Adiposity and Neuronal Behaviors
}

\author{
Abstract \\ The neural precursor cell expressed developmentally down-regulated gene 4 (NEDD4) is a HECT-type E3 \\ ubiquitin ligase that has received broad attention in recent years. Many of its reported substrates are \\ active players in metabolism, implying a potential role of NEDD4 itself in metabolic regulation. Since \\ homozygous Nedd4 deletion leads to embryonic or perinatal lethality, we investigated the function of \\ NEDD4 in metabolic regulation in vivo, using Nedd4- haploinsufficient mice in a high fat diet-induced \\ obesity (HFDIO) model. \\ Our studies show that Nedd4-haploinsufficient mice fed a normal diet (ND) exhibited decreased body \\ weight in both genders and proportionally reduced tissue mass compared to their age-matched wild-type \\ littermates. The heterozygotic Nedd4-deficient mice displayed moderate insulin resistance and mild \\ hyperinsulinemia. These findings are consistent with others' studies in which depletion of Nedd4 in \\ mouse embryonic fibroblasts (MEFs) led to a decline in insulinlike growth factor I (IGF-1) and insulin \\ signaling.
}

Surprisingly, Nedd4-haploinsufficient mice were partially protected against high-fat diet (HFD) induced obesity. Total weight gain was decreased by $30 \%$ in Nedd4-haploinsufficient mice on HFD, which was associated with reduced adiposity, improved insulin sensitivity in white adipose tissue (WAT), alleviated hepatic steatosis and attenuated lipid-induced inflammation. NEDD4 reduction did not affect serum glucose or lipid profile. The energy intake and physical activity were almost indistinguishable between the two groups. We present data indicating that these beneficial effects of Nedd4 haploinsufficiency under conditions promoting obesity or type 2 diabetes (T2D) derive from at least two mechanisms: 1) elevated levels of $\beta$-agonist induced lipolysis through stimulation of $\beta$-adrenergic signaling as evidenced by enhanced $\beta 2$ adrenergic receptor ( $\beta 2-A R$ ) cell surface levels; 2 ) repressed expression of a key adipogenic regulator, peroxisome proliferator-activated receptor gamma (PPARY) in Nedd4- haploinsufficient mice in the WAT. Downregulation of NEDD4 in 3T3-L1 preadipocytes impaired the adipogenic response, resulting in decreased PPARY expression. Thus, the reduced adiposity in Nedd4-haploinsufficient mice fed a HFD compared to littermate wild-type mice may be explained at least in part by their increased lipolysis and suppressed adipogenesis. Our data also imply that NEDD4 may have differential functions in modulating insulin signaling under physiological and pathological conditions.

In this study, we tested the possible impact of NEDD4 reduction on cognitive function and anxiety behaviors. Preliminary results showed that Nedd4-haploinsufficient mice had impaired learning and memory performance but increased anxiety compared to wild-type littermates. NEDD4 may modulate different behavioral functions by targeting specific substrates for protein degradation in a brain-regionspecific manner. Precise substrates that cause the alteration in such behaviors are yet to be determined by future studies.

\section{Document Type}

Dissertation

Degree Name

Doctor of Philosophy (PhD)

\section{Program}

Biomedical Sciences 


\section{Research Advisor}

Francesca-Fang Liao, Ph.D.

\section{Keywords}

Adipose tissue; Beta-adrenergic receptor; High-fat diet; Metabolic regulation; Obesity; PPAR gamma

\section{Subject Categories}

Diseases | Endocrine System Diseases | Genetic Processes | Medical Genetics | Medicine and Health Sciences 


\title{
EFFECT OF NEDD4 HAPLOINSUFFICIENCY ON INSULIN SENSITIVITY,
} ADIPOSITY AND NEURONAL BEHAVIORS

\author{
A Dissertation \\ Presented for \\ The Graduate Studies Council \\ The University of Tennessee \\ Health Science Center
}

\author{
In Partial Fulfillment \\ Of the Requirements for the Degree \\ Doctor of Philosophy \\ From The University of Tennessee
}

By

Jingjing Li

December 2014 
Copyright (C) 2014 by Jingjing Li. All rights reserved. 


\section{DEDICATION}

This dissertation is especially dedicated to my parents, Rihui Li and Zigui Fan, who have been supportive and encouraging in every aspect through my pursuit of

knowledge. The dedication is also given to my dear husband, Yongan Li, and my lovely son, Ryan Li for bringing balance and happiness into my life. 


\section{ACKNOWLEDGEMENTS}

First and foremost, I would like to thank my advisor, Dr. Francesca-Fang Liao for her sustained patience, endless support, constructive guidance and criticism during my Ph.D. training. I thank her not only for her wise mentorship, but also for her delicate considerateness and care in my every-day life.

I am deeply grateful to my other committee members for their encouraging and valuable comments: Dr. Suleiman W. Bahouth, Dr. Robert Ferry, Jr., Dr. Aviv I. Hassid (previous), Dr. Edwards A. Park, and Dr. Rajendra Raghow.

I would like to give special thanks to my former and present labmates, Bin Wang, Ruishan Wang, Lubin Lan, Yueqiang Xue, Eunhee Kim, Suzhen Gong, Shiyong Diao, Youngdon Kwak, Qiyue Deng, who have always been kind and friendly to me in work and in real life.

I would like to acknowledge Dr. Suleiman W. Bahouth for teaching me hands-on experimental skills, his valuable suggestions, and his generosity in sharing experimental equipment.

I would also like to thank Dr. Kui Li for teaching me important experimental skills in my first year of study, Dr. Bingzhong Xue (Georgia State University) for sharing her scientific expertise, and Dr. Jena J. Steinle for sharing reagents.

I would like to thank all of my friends in Memphis, especially my classmates, Hui $\mathrm{Yu}$, Shanshan $\mathrm{Yu}$, and $\mathrm{Xi}$ Wang for their help and advice.

Last but not least, I would like to thank my beloved family. I thank them for always being encouraging when I lost faith, for being supportive when I had limited free time, and for being tolerant when I lost my temper. They inspire me to become a better me. Without their support, I would not have come this far. 


\begin{abstract}
The neural precursor cell expressed developmentally down-regulated gene 4 (NEDD4) is a HECT-type E3 ubiquitin ligase that has received broad attention in recent years. Many of its reported substrates are active players in metabolism, implying a potential role of NEDD4 itself in metabolic regulation. Since homozygous Nedd4 deletion leads to embryonic or perinatal lethality, we investigated the function of NEDD4 in metabolic regulation in vivo, using Nedd4-haploinsufficient mice in a high fat dietinduced obesity (HFDIO) model.

Our studies show that Nedd4-haploinsufficient mice fed a normal diet (ND) exhibited decreased body weight in both genders and proportionally reduced tissue mass compared to their age-matched wild-type littermates. The heterozygotic Nedd4-deficient mice displayed moderate insulin resistance and mild hyperinsulinemia. These findings are consistent with others' studies in which depletion of Nedd4 in mouse embryonic fibroblasts (MEFs) led to a decline in insulin-like growth factor I (IGF-1) and insulin signaling.
\end{abstract}

Surprisingly, Nedd4-haploinsufficient mice were partially protected against highfat diet (HFD) induced obesity. Total weight gain was decreased by $30 \%$ in Nedd4haploinsufficient mice on HFD, which was associated with reduced adiposity, improved insulin sensitivity in white adipose tissue (WAT), alleviated hepatic steatosis and attenuated lipid-induced inflammation. NEDD4 reduction did not affect serum glucose or lipid profile. The energy intake and physical activity were almost indistinguishable between the two groups. We present data indicating that these beneficial effects of Nedd4 haploinsufficiency under conditions promoting obesity or type 2 diabetes (T2D) derive from at least two mechanisms: 1 ) elevated levels of $\beta$-agonist induced lipolysis through stimulation of $\beta$-adrenergic signaling as evidenced by enhanced $\beta 2$ adrenergic receptor $\left(\beta_{2}\right.$-AR) cell surface levels; 2 ) repressed expression of a key adipogenic regulator, peroxisome proliferator-activated receptor gamma (PPAR $\gamma$ ) in Nedd4-haploinsufficient mice in the WAT. Downregulation of NEDD4 in 3T3-L1 preadipocytes impaired the adipogenic response, resulting in decreased PPAR $\gamma$ expression. Thus, the reduced adiposity in Nedd4-haploinsufficient mice fed a HFD compared to littermate wild-type mice may be explained at least in part by their increased lipolysis and suppressed adipogenesis. Our data also imply that NEDD4 may have differential functions in modulating insulin signaling under physiological and pathological conditions.

In this study, we tested the possible impact of NEDD4 reduction on cognitive function and anxiety behaviors. Preliminary results showed that Nedd4-haploinsufficient mice had impaired learning and memory performance but increased anxiety compared to wild-type littermates. NEDD4 may modulate different behavioral functions by targeting specific substrates for protein degradation in a brain-region-specific manner. Precise substrates that cause the alteration in such behaviors are yet to be determined by future studies. 


\section{TABLE OF CONTENTS}

CHAPTER 1. INTRODUCTION ................................................................................

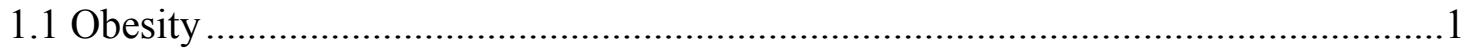

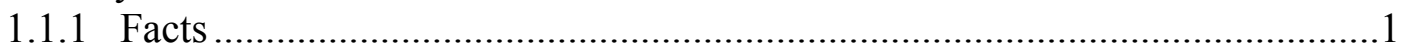

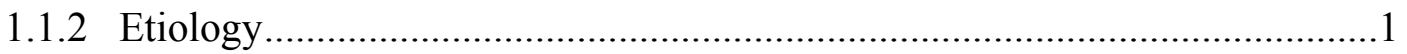

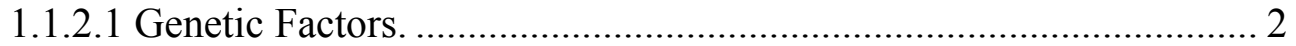

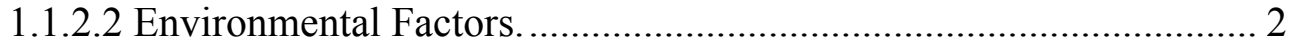

1.1.2.3 Psychological Factors. ................................................................ 2

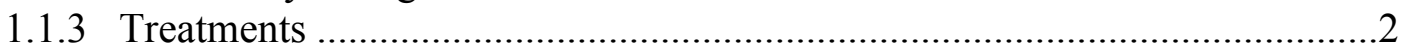

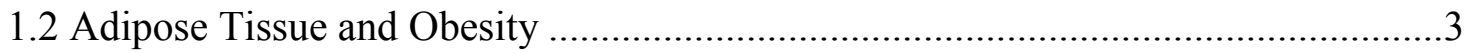

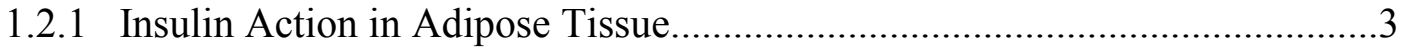

1.2.2 Insulin Resistance in Adipose Tissue .................................................

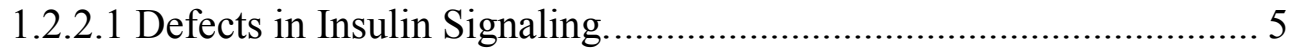

1.2.2.2 Increased Release of FFAs. ....................................................... 5

1.2.2.3 Changed Adipokine Profile. .............................................................. 6

1.2.3 Lipid Storage and Mobilization in Adipose Tissue ...................................

1.2.4 Different Adipose Tissue Depots............................................................... 7

1.2.5 Hypertrophy and Hyperplasia of Adipose Tissue .......................................10

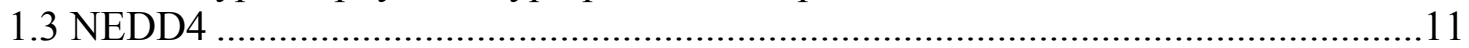

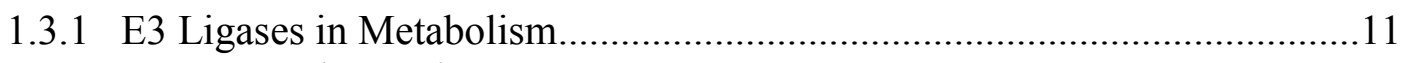

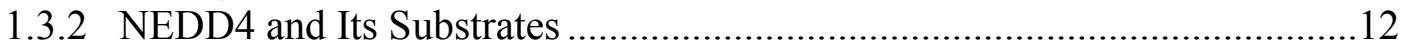

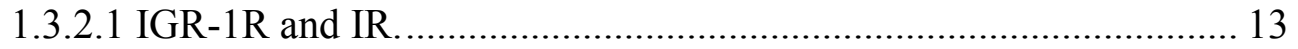

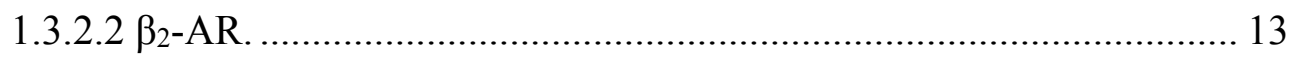

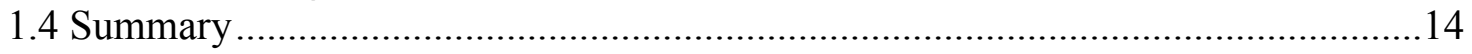

CHAPTER 2. MATERIALS AND METHODS....................................................16

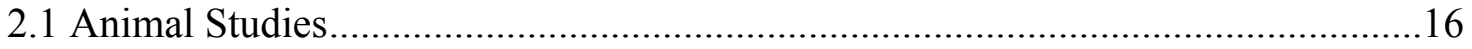

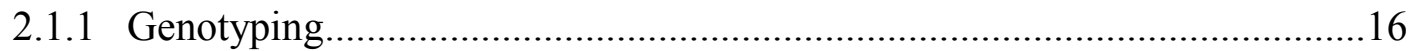

2.1.2 Food Intake, Body Weight, and Body Length Measurement ......................16

2.1.3 Glucose Tolerance Test (GTT) and Insulin Tolerance Test (ITT) ...............16

2.1.4 Insulin Signaling ................................................................................ 17

2.1.5 Measurement of Metabolites and Hormones ............................................ 17

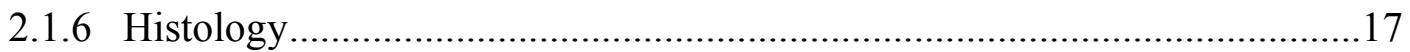

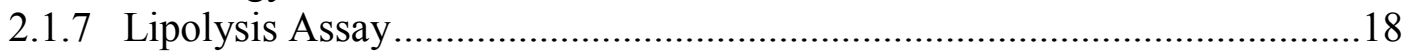

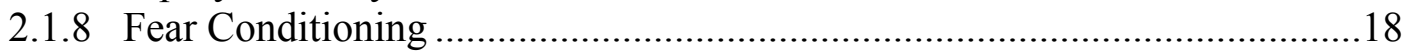

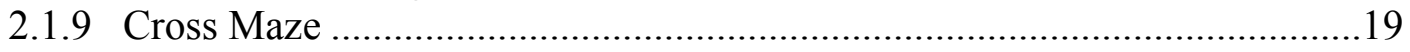

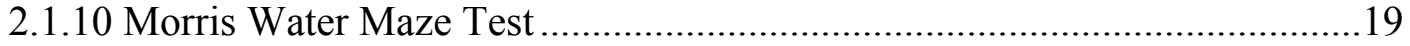

2.1.11 Open Field Test................................................................................ 19

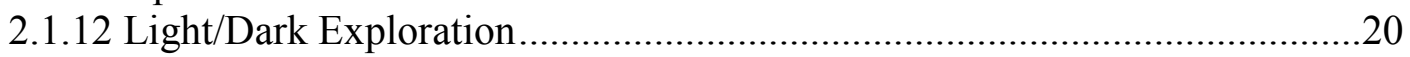

2.2 Biochemical Studies ....................................................................................... 20

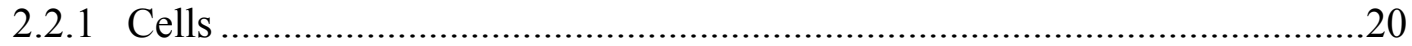

2.2.2 Adeno-associated Virus (AAV)-mediated Knockdown ............................20

2.2.3 Western-blot Analysis.........................................................................21

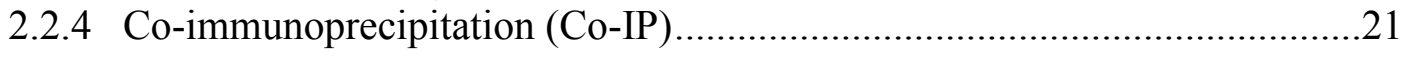


2.2.5 RNA Isolation, cDNA Synthesis, and qRT-PCR ………………..............21

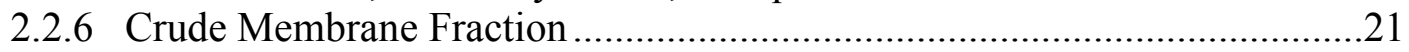

2.2.7 Oil Red O Staining in Differentiated 3T3-L1 Cells....................................23

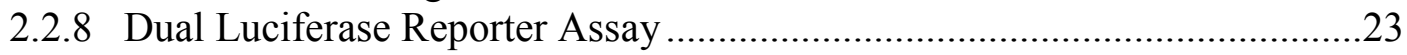

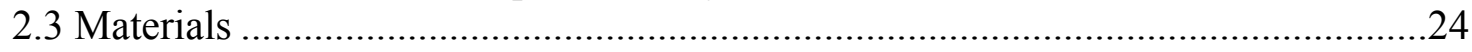

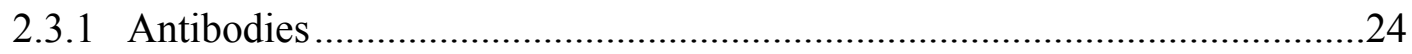

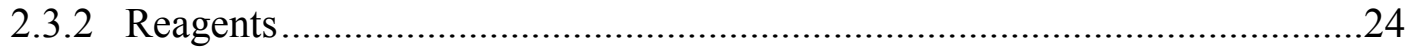

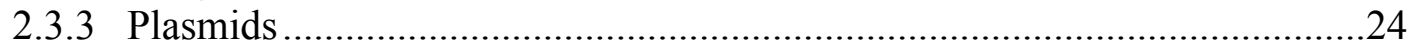

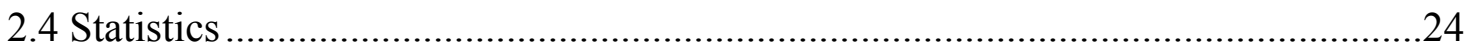

CHAPTER 3. METABOLIC PHENOTYPE STUDIES .............................................25

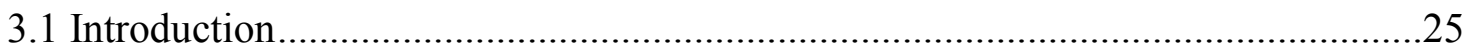

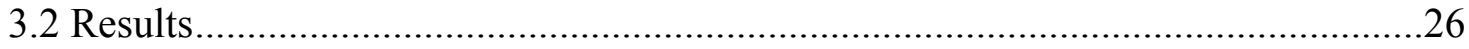

3.2.1 Genotype Verification of Nedd4-haploinsufficient Mice .............................26

3.2.2 Nedd4-haploinsufficient Mice Resist HFD-induced Obesity ........................26

3.2.3 Nedd4-haploinsufficient Mice Are Resistant to Several HFD-induced Deleterious Effects without Changes in Serum Lipid Profile .......................29

3.2.4 Nedd4-haploinsufficient Mice Retain Insulin Sensitivity in WAT despite

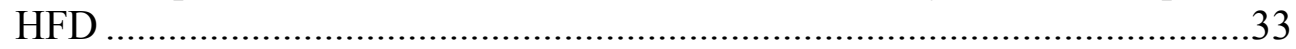

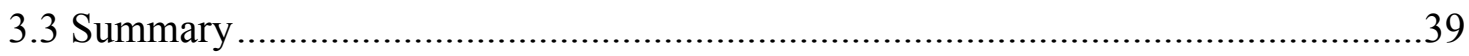

CHAPTER 4. MECHANISTIC STUDIES.....................................................................43

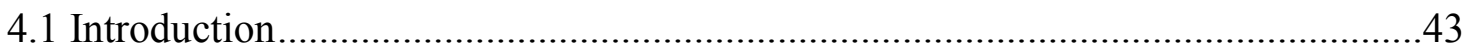

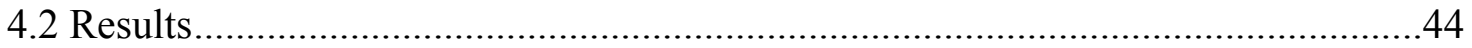

4.2.1 Knockdown of NEDD4 Accelerates Lipolysis ..............................................44

4.2.2 Knockdown of NEDD4 Increases $\beta_{2}$-AR Abundance at the Plasma

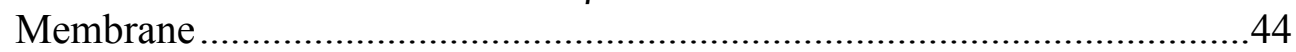

4.2.3 Knockdown of NEDD4 Increases Lipolytic but Inhibits Adipogenic

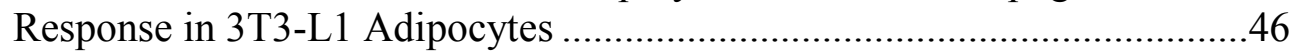

4.2.4 Knockdown of NEDD4 Destabilizes PPAR $\gamma$ Protein.....................................49

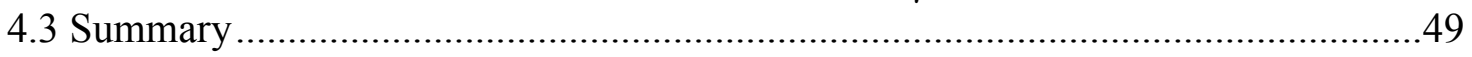

CHAPTER 5. NEUROBEHAVIORAL ANALYSES..................................................56

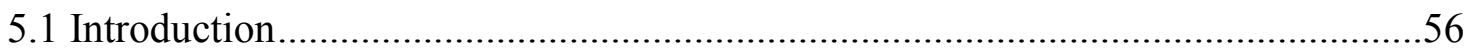

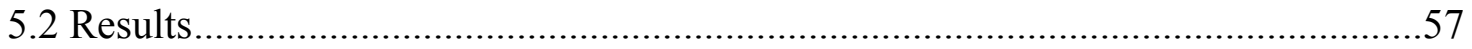

5.2.1 Nedd4-haploinsufficient Mice Have Improved Fear-associated Memory....57

5.2.2 Nedd4-haploinsufficient Mice Display More Spontaneous Alternation........57

5.2.3 Nedd4-haploinsufficient Mice Display Impaired Water Maze

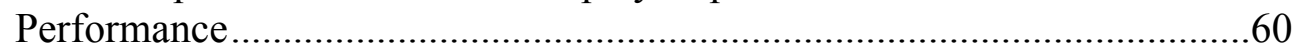

5.2.4 Nedd4-haploinsufficient Mice Display Anxiety-like Behavior ......................60

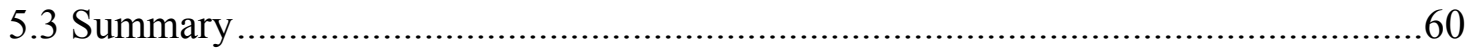

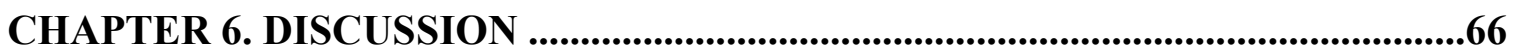

6.1 Pathophysiological Roles of NEDD4 in Obesity.................................................66

6.1.1 The Energy Balance in Nedd4-haploinsufficient Mice..................................67 
6.1.2 Peripheral Insulin Sensitivity in Nedd4-haploinsufficient Mice..................69

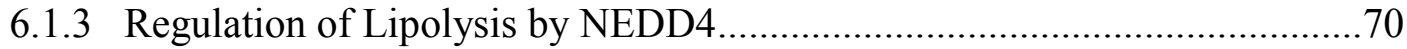

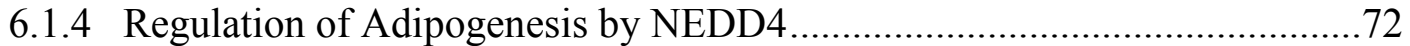

6.1.5 E3 Ligases in Metabolism..................................................................... 73

6.2 Physiological Role of NEDD4 in CNS .......................................................... 76

6.2.1 Nedd4 Haploinsufficiency Leads to Altered Cognitive and Anxiety-like

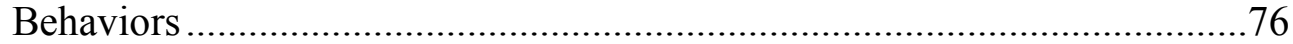

6.2.2 Central Insulin Signaling in Nedd4-haploinsufficient Mice .......................76

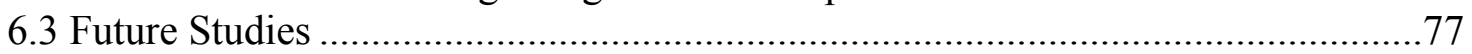

6.4 Using Heterozygous Mouse as Models_-Advantages vs. Disadvantages...............78

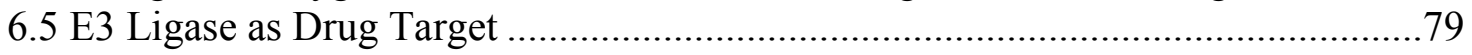

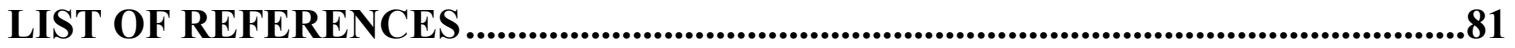

APPENDIX: SUPPLEMENTAL INFORMATION.......................................................99

VITA 


\section{LIST OF TABLES}

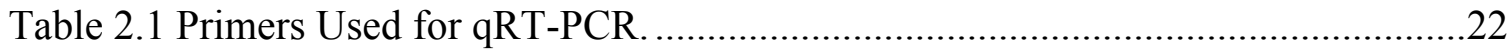

Table 3.1 Metabolic Parameters in Serum or Blood........................................................37 


\section{LIST OF FIGURES}

Figure 1.1 Lipid Metabolism in Adipocyte by $\beta$-adrenergic and Insulin Signaling. .........8

Figure 1.2 Regulation of $\beta_{2}$-AR Endocytosis and Degradation by NEDD4 ....................15

Figure 3.1 PCR and Western-blot Confirmation of Genotypes........................................27

Figure 3.2 Nedd4-haploinsufficient Mice on HFD Exhibit Less Weight Gain. ...............28

Figure 3.3 Nedd4-haploinsufficient Mice Exhibit Normal Physical Activity, Energy

Intake, and Thermogenesis-related Gene Expression......................................30

Figure 3.4 Nedd4-haploinsufficient Mice on HFD Have Reduced Fat Mass....................31

Figure 3.5 Nedd4-haploinsufficient Mice Are Protected against HFD-induced Adipocyte Hypertrophy. ....................................................................................

Figure 3.6 Nedd4-haploinsufficient Mice Are Protected against HFD-induced

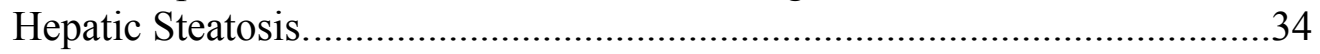

Figure 3.7 Nedd4-haploinsufficient Mice Are Protected against Lipid-induced

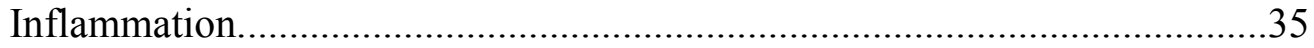

Figure 3.8 NEDD4 Protein Levels Are Not Altered by HFD Feeding.............................36

Figure 3.9 GTT, ITT, and Serum Insulin Levels........................................................38

Figure 3.10 In vivo Insulin Signaling in WAT, Skeletal Muscle, and Liver. .....................40

Figure 4.1 Downregulation of NEDD4 Enhances Hormone-stimulated Lipolysis In

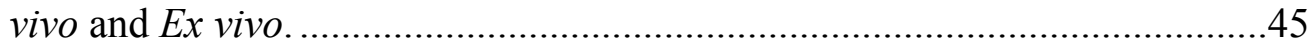

Figure 4.2 Loss of NEDD4 Function Increases $\beta 2$-AR Protein Concentration at Plasma Membrane.

Figure 4.3 Partial Loss of NEDD4 Function Increases Lipolytic but Inhibits Adipogenic Response.

Figure 4.4 Partial Loss of NEDD4 Function Reduces PPAR $\gamma$ Levels in WAT. ..............50

Figure 4.5 NEDD4 Has No Direct Effect on PPAR $\gamma$ Activity. ..........................................51

Figure 4.6 NEDD4 Stabilizes PPAR $\gamma$ Protein Expression In vitro. .................................52

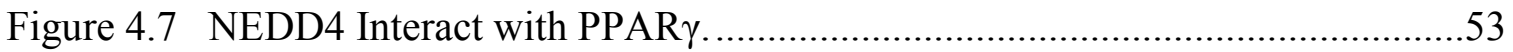


Figure 4.8 NEDD4-PPAR $\gamma$ Interaction Alteration during Adipocyte Conversion in 3T3-L1 Cells.

Figure 5.1 Improved Fear-associated Memory in Nedd4-haploinsufficient Mice...........58

Figure 5.2 Improved Learning and Memory in Cross Maze Test in Nedd4haploinsufficient Mice.

Figure 5.3 Impaired Learning and Memory in Morris Water Maze Test in Nedd4haploinsufficient Mice.

Figure 5.4 Partial Loss of NEDD4 Reduces Insulin-stimulated IR

Autophosphorylation.

Figure 5.5 Locomotor Activity Test Indicates Anxiety-like Behavior in Nedd4haploinsufficient Mice.

Figure 5.6 Light/dark Box Test Indicates Anxiety-like Behavior in Nedd4haploinsufficient Mice.

Figure 6.1 Schematic Diagram of NEDD4 Regulation of Lipolysis and Adipogenesis.

Figure A.1 AMPK, mTOR, and Leptin Signaling in Hypothalamus of Nedd4haploinsuffcient Mice. 


\section{CHAPTER 1. INTRODUCTION}

\subsection{Obesity}

\subsubsection{Facts}

The World Health Organization (WHO) defines overweight and obesity as a state of "abnormal or excessive fat accumulation" (WHO official website). According to the statistics provided by the $\mathrm{WHO}$, there are 500 million adults worldwide who are obese with a body mass index (BMI) over $30 \mathrm{~kg} / \mathrm{m}^{2}$ and one billion who are overweight with a BMI over $25 \mathrm{~kg} / \mathrm{m}^{2}$. The number of overweight children under the age of five is escalating each year exceeding 40 million in 2012 (Ogden et al., 2012). A survey conducted by the United States Centers for Disease Control and Prevention (USCDC) shows that Tennessee is among the states with highest obesity incident rate, having a prevalence greater than 30\% (2008) .

Increasingly recognized as disorders in their own right, being overweight or obese certainly adversely affects individual's quality of life. Moreover, an emerging body of scientific, medical, and behavioral studies suggest raised body fat as a major risk factor for the development of metabolic syndrome, T2D, cardiovascular disease (mainly heart disease and stroke), and certain types of cancer. In the United States alone, obesity and its complications contribute to over 300,000 premature deaths each year, making it the second preventable cause of death behind tobacco smoking (Mokdad et al., 2004).

\subsubsection{Etiology}

Causes of obesity include genetic predisposition, environmental and psychological factors. However, ultimately, obesity is a consequence of chronic imbalance between energy intake and output. In certain cases, energy required for growth and energy utilized for basic metabolic processes also account for the energy balance. When energy intake exceeds energy output, a state of positive energy balance occurs, thereby increasing body weight (Hill et al., 2012). After linear growth in early adulthood energy balance becomes determined by three basic components: energy intake, energy output, and energy storage (Hill et al., 2003).

Humans absorb energy in the forms of protein, carbohydrate and fat. Humans expend energy primarily through basal metabolic rate, physical activity, and diet-induced thermogenesis. The basal metabolic rate is the rate of energy expenditure at rest, which is proportional to body mass and depends essentially on the lean mass (mostly the muscles) (White and Seymour, 2003). The basal metabolic rate represents $60-70 \%$ of the total energy output (Blundell et al., 2012). The energy expended through physical activity varies and accounts for $20-30 \%$ of total energy output (Rising et al., 1994). Diet-induced 
thermogenesis is an increase in metabolic rate that occurs after eating, representing approximately $10 \%$ of the total energy output (Tappy et al., 1991).

1.1.2.1 Genetic Factors. Genetic factors may cause changes in energy intake. For example, mutations in leptin (Montague et al., 1997; Strobel et al., 1998), its receptor (Clement et al., 1998), or mutations in melanocortin receptor 4 (Vaisse et al., 1998; Yeo et al., 1998), proopiomelanocortin (Krude et al., 1998) and prohormone convertase 1 (Jackson et al., 1997) lead to pronounced hyperphagia and obesity. Energy expenditure and energy storage can be regulated by genetic factors as well. For example, mutations in uncoupling protein-2 (Fleury et al., 1997), acyl CoA:diacylglycerol transferase (Smith et al., 2000), perilipin (Martinez-Botas et al., 2000), or cell death activator (cidea) (Zhou et al., 2003) can lead to corresponding metabolic modifications. Recent genome wide association studies and bioinformatics analysis have identified numerous single nucleotide polymorphisms (SNPs) and loci which are strongly associated with body fatness. Many of these SNPs lay within introns or even in the noncoding regions. Though many monogenic effects have been identified, multiple subtle variants in genes are likely to contribute to the common forms of human obesity, as is the case with T2D (Farooqi and O'Rahilly, 2007).

1.1.2.2 Environmental Factors. Contemporary environments are filled with readily available, inexpensive foods with high calorie content facilitating obesity. Advanced technology and transportation systems promote sedentary life styles which are frequently linked to the epidemic of obesity (Hill and Peters, 1998).

1.1.2.3 Psychological Factors. Obesity can be caused by psychological factors as well. People with psychological disorders (e.g. depression, anxiety, low self-esteem, or other emotions) may eat aberrantly, exercise insufficiently, and therefore, are more likely to develop obesity (Stice et al., 2005).

\subsubsection{Treatments}

Balanced eating and healthy lifestyle are important strategies for weight loss and maintenance. Weight loss helps improve overall health and reduce risk of developing cardiovascular complications. For treatment of obesity, the commercially available drugs mainly act through two mechanisms: reduce fat absorption through inhibition of gastric and pancreatic lipases (e.g. orlistat (Curran and Scott, 2004)) and suppress appetite (e.g. phentermine/ topiramate and lorcaserin (Fleming et al., 2013)). 


\subsection{Adipose Tissue and Obesity}

Adipose tissue is the main storage site for excess energy in the form of triglycerides (TGs). Besides its storage function, adipose tissue also serves as an endocrine organ, which is able to secrete a variety of cytokines, including leptin. Adipose tissue is emerging as an important component in metabolism. An adipocentric view even considers adipose as a determinant factor for insulin resistance. Indeed, excessive adipose tissue increases the risk for the development of insulin resistance. Surprisingly, a lack of adipose tissue in the body, for example in the case of Berardinelli-Seip congenital lipodystrophy, in which fats are stored at ectopic sites in the body, such as in the liver and muscles, can also lead to severe insulin resistance (Magre et al., 2001). A transgenic model of inducible lipoatrophy provides consistent evidence (Pajvani et al., 2005). While these observations seem paradoxical at first sight, they imply that proper function of adipose tissue is critical for maintaining whole-body insulin sensitivity. A brief introduction to the concepts of insulin action and insulin resistance is necessary in order to understand these observations.

\subsubsection{Insulin Action in Adipose Tissue}

Insulin is a small molecule of 51-residue and with a molecular weight of approximately 6 kilodalton $(\mathrm{kDa})$. It is produced by the pancreatic $\beta$ cells, when amino acids or glucose levels are increased in the bloodstream. Tissues that are responsive to insulin action are referred to as insulin-sensitive tissues, which include but are not limited to skeletal muscle, liver, fat and hypothalamus.

The cellular insulin signaling event initiates from the membrane-localized insulin receptor (IR), which belongs to the family of receptor tyrosine kinases (RTKs). The IR is a heterotetramer consisting of two $\alpha$-subunits and two $\beta$-subunits held together by disulfide and noncovalent bonds (Lee and Pilch, 1994). IR undergoes glycosylation before its insertion to the plasma membrane. Both subunits are exposed to the extracellular environment, while only the C-terminal $\beta$-subunit is intracellular (Lee and Pilch, 1994). Following ligand binding, the $\beta$-subunit undergoes autophosphorylation on seven Tyr residues in a sequential order and a conformational change that further increases kinase activity. The three most critical tyrosine sites are Tyr 1158, 1162 and 1163, phosphorylation of which results in activation of the receptor kinase activity (Feener et al., 1993; Hubbard, 1997). Subsequently, the activated IR phosphorylates a number of intracellular substrates on tyrosine. The primary substrates are the members of the insulin receptor substrate family (IRS1/2/3/4). Other substrates include: Srchomology-2-containing protein (Shc), IRS-5 and IRS-6 (or DOK-4 and DOK-5), Cas-Br$\mathrm{M}$ (murine) ectopic retroviral transforming sequence homologue $(\mathrm{Cbl})$, Cbl-associated protein (CAP), signal transducer and activator of transcription (STAT) and GRB2associated-binding protein 1 (Gab-1), and p62dok (Cai et al., 2003; Lehr et al., 2000; Taniguchi et al., 2006; Wick et al., 2001). 
Following phosphorylation, these substrates serve as important intermediates that interact with other intracellular molecules. For example, tyrosine phosphorylation of IRS proteins creates recognition sites for other proteins containing Src homology 2 (SH2) domains, most notably, the p85 regulatory subunit of the phosphatidylinositol 3-kinase

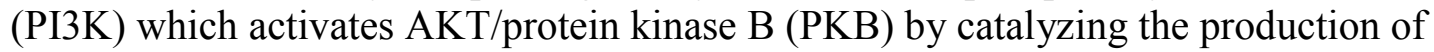
phosphatidylinositol-3,4,5-triphosphate $\left(\mathrm{PIP}_{3}\right)$ from phosphatidylinositol 4,5bisphosphate ( $\left.\mathrm{PIP}_{2}\right)$ at the plasma membrane (Taniguchi et al., 2006). This eventually promotes the two main downstream signaling pathways: the PI3K-AKT/PKB pathway and the Ras-mitogen-activated protein kinase (Ras-MAPK) pathway. The former pathway mediates most of insulin's metabolic actions, while the latter pathway regulates general gene expressions for cell growth, survival, and differentiation (Taniguchi et al., 2006).

$\mathrm{PI} 3 \mathrm{~K}$ is necessary for insulin-stimulated glucose transport via AKT/PKB (Wang et al., 1999b) and/or the atypical protein kinase $\mathrm{C}$ (PKC) isoform $\lambda / \zeta($ Kotani et al., 1998), whereas another pathway involving the Cbl-CAP complex is also implicated for the translocation of glucose transporter type 4 (GLUT4) protein from intracellular sites to the cell surface of the membrane (Chiang et al., 2001).Besides stimulation of glucose uptake, insulin is also known to be an important antilipolytic hormone in adipose tissue. Insulin exerts its antilipolytic effect through inducing phosphorylation and activation of the phosphodiesterase type 3B (PDE3B), which is dependent on the activation of PI3K/AKT pathway (Kitamura et al., 1999; Rondinone et al., 2000). Activation of PDE3B decreases concentrations of adenosine 3',5'-monophosphate (cAMP), which in turn reduces the activity of protein kinase A (PKA), a cAMP-dependent protein kinase (Degerman et al., 1997). PKA is responsible for activation of two important proteins with established function in lipolysis: the hormone-sensitive lipase (HSL), an enzyme that catabolizes TGs in adipocytes, and perilipin A, a protein that associates with the surface of lipid droplets and the phosphorylation of which is required for lipid droplet mobilization (Zimmermann et al., 2004). It should be noted those insulin concentrations required for its antilipolytic effect are lower than that required for its glucose uptake effect. Hence, even under insulin resistant conditions when the insulin-stimulated glucose uptake is impaired, its antilipolytic effect maintains the growth of adipose tissue (Kahn and Flier, 2000). In addition, insulin serves as a potent pro-adipogenic hormone that triggers the expression of a series of transcription factors facilitating the conversion from pre-adipocytes to mature adipocytes (Rosen and MacDougald, 2006).

\subsubsection{Insulin Resistance in Adipose Tissue}

Insulin resistance is a physiological state where the body fails to respond adequately to insulin. This is manifested by reduced glucose uptake in adipocytes and skeletal muscle and by diminished suppression of hepatic glucose production. In the 1930s, Dr. H.P. Himsworth was the first to distinguish insulin resistance from insulin deficiency (Himsworth, 1940). He found that some diabetic individuals (typically lean and with early-onset diabetes) could rapidly respond to the injection of insulin, while some others (obese and with later-onset diabetes) could not. Following this track, in the 
1960s, Dr. D. Rabinowitz and K.L. Zierler discovered that some obese but non-diabetic individuals were also resistant to the effects of insulin (Rabinowitz and Zierler, 1961). An extensive clinical study in 1990s provided the most direct evidence that the insulin sensitivity index is negatively correlated with abdominal fatness in both men and women (Clausen et al., 1996). Normally, the more fat tissue one has the more resistant his or her cells respond to insulin. Therefore, insulin resistance is commonly seen coexisting with obesity. It is now well accepted that obesity-associated insulin resistance is the major mechanistic basis for the link between obesity and many other metabolic diseases, such as T2D and cardiovascular disease (Kahn and Flier, 2000).

Insulin resistance in obesity is hypothesized to be caused by defects at multiple levels of insulin signaling, increased amount of non-esterified fatty acids (NEFAs), also referred to as free fatty acids (FFAs), released from adipose tissue and changed adipokine profile.

1.2.2.1 Defects in Insulin Signaling. Several lines of evidence suggest that insulin signaling in insulin-responsive tissues is impaired in obesity. In skeletal muscles from obese humans, expression of several insulin signaling molecules was decreased (Goodyear et al., 1995). In obese humans with T2D, IRS-1 expression was found to be reduced in adipocytes (Rondinone et al., 1997), and PI3K activity was attenuated in both adipocytes and skeletal muscles (Kim et al., 1999; Rondinone et al., 1997). Mice double knockouts of IRS-1 and IRS-3 exhibited a severe phenotype: severe lipoatrophy at early stage and insulin resistance (Laustsen et al., 2002). The expression and activity of several protein tyrosine phosphatases (PTPs), such as PTP1B and src-homology-containingphosphatase 2 (SHP-2) were increased in skeletal muscle and adipose tissue in obese humans and rodents (Goldstein et al., 1998). PTPs are negative regulators of insulin signaling by dephosphorylating IR. In agreement, PTP1B knockout mice had increased insulin sensitivity and resistance to diet-induced obesity (Elchebly et al., 1999; Klaman et al., 2000).

Surprisingly, the fat insulin receptor knockout (FIRKO) mice displayed improved metabolic outcomes compared to wild-type mice (Bluher et al., 2002). When the mice were fed with a high-fat diet (HFD) or were treated with gold thioglucose, which caused hypothalamic damage and hyperphagia, the beneficial effects of the loss of insulin signaling in fat became even more significant. The FIRKO mice had reduced fat mass. They were protected against obesity and its associated glucose intolerance (Bluher et al., 2003; Bluher et al., 2002). Moreover, both male and female FIRKO mice had an increase in average life-spans (Bluher et al., 2003). Therefore, reduced insulin signaling in adipocytes, nonetheless, appears to prevent the development of obesity and its associated metabolic abnormalities.

1.2.2.2 Increased Release of FFAs. Elevated FFA concentrations in obesity are thought to arise from the expanded adipose tissue. FFA has long been recognized as an energy source for other tissues. An additional function of FFA as an endocrine factor that 
regulates metabolic functions has been proposed since 1960s (Kennedy, 1992).Moreover, FFA has been proposed as a major culprit for insulin resistance: under insulin resistant state, lipolysis in adipocyte which is normally suppressed by insulin, is further increased, thereby liberating more FFA and in turn aggravating insulin resistance. In this model, elevated FFA levels are demonstrated to lead to intracellular accumulation of several metabolites including acyl-CoAs, ceramides, and diacyglycerol (DAG), which can activate protein kinases such as PKC, c-Jun N-terminal kinases (JNKs), and the inhibitor of nuclear factor- $\kappa \mathrm{B}(\mathrm{NF}-\kappa \mathrm{B})$ kinase- $\beta$ (IKK $\beta)$. These kinases in turn increases the inhibitory serine phosphorylation of IRS1/2 (Petersen and Shulman, 2006).

However, this concept has now been questioned. In a clinical study involving a prospective cohort of 481 women and 345 men, the fasting FFA concentrations did not predict the development of insulin resistance (Byrne et al., 1999). In the Karpe et al. study, in which 43 original reports including a total number of 953 non-obese (control) patients and 1,410 overweight/obese patients were pooled and reanalyzed in aggregates by literature search from PubMed and were aligned for a relationship between the fasting FFA concentration and the fat mass. Surprisingly, the result clearly showed a complete absence of relationship between adipose tissue mass and the fasting FFA concentration (Karpe et al., 2011). Therefore, whether the elevated FFA levels truly contribute to the pathogenesis of obesity-associated insulin resistance remains an open question.

1.2.2.3 Changed Adipokine Profile. The profile of adipokines secreted from adipose tissue also undergoes major changes in obesity. Release of most adipokines is elevated in obesity, including the metabolically beneficial hormone leptin (Sinha et al., 1996). Leptin signaling is concomitantly impaired in obesity(Jequier, 2002). Loss of leptin produced severe obesity in a previous study (Montague et al., 1997). Resistin, an adipocyte-secreted protein, was also found to circulate at high levels in the bloodstream in obese rodents. Infusion or sustained overexpression of resistin caused insulin resistance, while knocking out resistin in mice improved glucose metabolism (Steppan et al., 2001). Conversely, adiponectin levels are low in obesity. The administration or transgenic overexpression of adiponectin improved insulin resistance and lowered FFAs in serum in animal models (Lihn et al., 2005). Consistently, adiponectin-deficient mice developed insulin resistance and had increased serum FFAs (Lihn et al., 2005).

Highly upregulated adipokines in adipose tissue of obese subjects also include several proinflammatory cytokines. For example, the plasma concentration of monocyte chemoattractant protein 1 (MCP-1) was high in obese mouse models, leading to massive macrophage infiltration and insulin resistance (Kanda et al., 2006). Other inflammationassociated cytokines released from macrophages or adipocytes are tumor necrosis factor $\alpha(\mathrm{TNF} \alpha)$, interleukin-1 $\beta$ (IL-1 $\beta$ ) and interleukin-6 (IL-6) (Qatanani and Lazar, 2007). It has been proposed that cytokines released from adipose tissue induce a systemic lowgrade inflammation and promote insulin resistance (Xu et al., 2003). 


\subsubsection{Lipid Storage and Mobilization in Adipose Tissue}

Adipocytes synthesize TGs from circulating FFA pool. After a meal, circulating dietary fat absorbed by the intestinal tract in the form of chylomicrons is hydrolyzed by lipoprotein lipase (LPL) in the capillary endothelium in adipose tissue. FFAs thus released are taken up into the adipocytes for storage. The endogenous TGs carried by very low-density lipoprotein (VLDL) are removed by LPL from VLDL and taken up by adipocytes via VLDL receptor (VLDL-R) or low density lipoprotein receptor-related protein (LRP) for storage. FFAs can also be transported into adipocytes via a family of fatty acid transport proteins (FATPs), such as FATP1 (Gimeno, 2007). Fatty acids are transported by fatty acid binding proteins (FABPs) inside an adipocyte, notably the FABP-4 (Maeda et al., 2005). After their entry, fatty acids are esterified by acyl-coA synthetase. Esterified fatty acids together with glycerol are used for TG synthesis. Alternatively, adipocytes can use acetyl-CoA for de novo fatty acid synthesis via acetylCoA carboxylase (ACC) and fatty acid synthase (FAS). TG are incorporated into lipid droplets and covered by perilipin. The remnant FFAs and TG-rich lipoproteins that are not taken up by the adipocytes are eventually taken up in ectopic sites, such as skeletal muscles, liver, pancreas, and heart (Karpe et al., 2011).

FFAs are stored as TG in a highly dynamic fashion. When necessary, FFAs can be re-released from fat. Break down of TGs to glycerol and FFAs is called lipolysis. During energy deprivation (e.g. fasting and exercise), lipolysis is stimulated in adipocytes and leads to the liberation of FFAs, which can be metabolized by other tissues, such as muscle, for energy production via $\beta$-oxidation (Duncan et al., 2007). The process of TG hydrolysis is three steps: TG is first to form diacylglycerol (DAG) by adipose triglyceride lipase (ATGL), then to monoacylglycerol (MAG) by HSL, and finally to FFAs and glycerol by monoacylglycerol lipase (MGL). HSL is the rate-limiting as well as the hormonally regulated step of this process. The lipolytic response is mainly controlled by catecholamines (epinephrine and norepinephrine) and the fasting hormones glucagon, via their binding to respective receptors, i.e. adrenergic receptors and glucagon receptors. Both lead to activation of adenylate cylase by stimulatory guanine nucleotide regulatory proteins (Gs) within the plasma membrane. Adenylate cyclase then enzymatically converts ATP to cAMP, which in turn activates PKA by binding to its regulatory subunit and releasing its catalytic subunit. Subsequently, the catalytic subunit of PKA phosphorylates perilipin and HSL for fat mobilization (Figure 1.1). While fasting or exercise enhance lipolysis, defective lipolysis was found in mature fat cells and in differentiated preadipocytes isolated from obese and non-obese human (Langin et al., 2005). Therefore, modulation of adipocyte lipolysis via pharmacological intervention may be a promising therapeutic strategy for treating obesity.

\subsubsection{Different Adipose Tissue Depots}

Classically, there are two types of adipose tissues within body: white and brown. It has long been believed that only newborns humans and rodents have interscapular brown adipose tissue (BAT). However, several recent studies show that brown adipocytes 
Figure 1.1 Lipid Metabolism in Adipocyte by $\beta$-adrenergic and Insulin Signaling. Adipocyte express all three $\beta$-AR subtypes - $\beta_{1}, \beta_{2}$, and $\beta_{3}$. Catecholamine binds to the $\beta$ AR and initiates a dissociation of Gas from G $\beta \gamma$. Gas stimulates the membrane associated enzyme adenylate cyclase (AC) to promote the production of cAMP from ATP and subsequently, activates PKA. Activated PKA phosphorylates both perilipin-1 and HSL. Phosphorylated perilipin-1 releases 1-acylglycerol-3-phosphate O-acyltransferase (ABHD5) (or CGI-58) to ATGL. Upon ABHD5 binding, ATGL is activated and initiates lipolysis of TG to produce DAG and FFA. PKA-activated HSL further hydrolyzes DAG to MAG and FFA. MAG is finally hydrolyzed to glycerol and FFA by MGL. Glycerol and FFA can be transported outside of the adipocyte by their respective transporter proteins, while FFA can also diffuse through plasma membrane. Insulin binding to IR on the surface of adipocyte activates PDE through PI3K/AKT pathway. PDE inhibits the production of cAMP and consequently reduces the activity of PKA. On the other hand, insulin promotes the translocation of GLUT4 to cell membrane and facilitates glucose uptake into adipocyte which is converted to glycerol-3-phosphate (G3P) through glycolysis. Insulin also activates LPL on adipocyte surface. Activated LPL releases FFA from lipoprotein which is subsequently transported or diffuses into adipocyte.

Intracellularly, FFA is converted to acyl-CoA. G3P and acyl-CoA serves as substrates for synthesis of TG at ER. PA, phosphatidic acid; LPA, lysophosphatidic acid. 


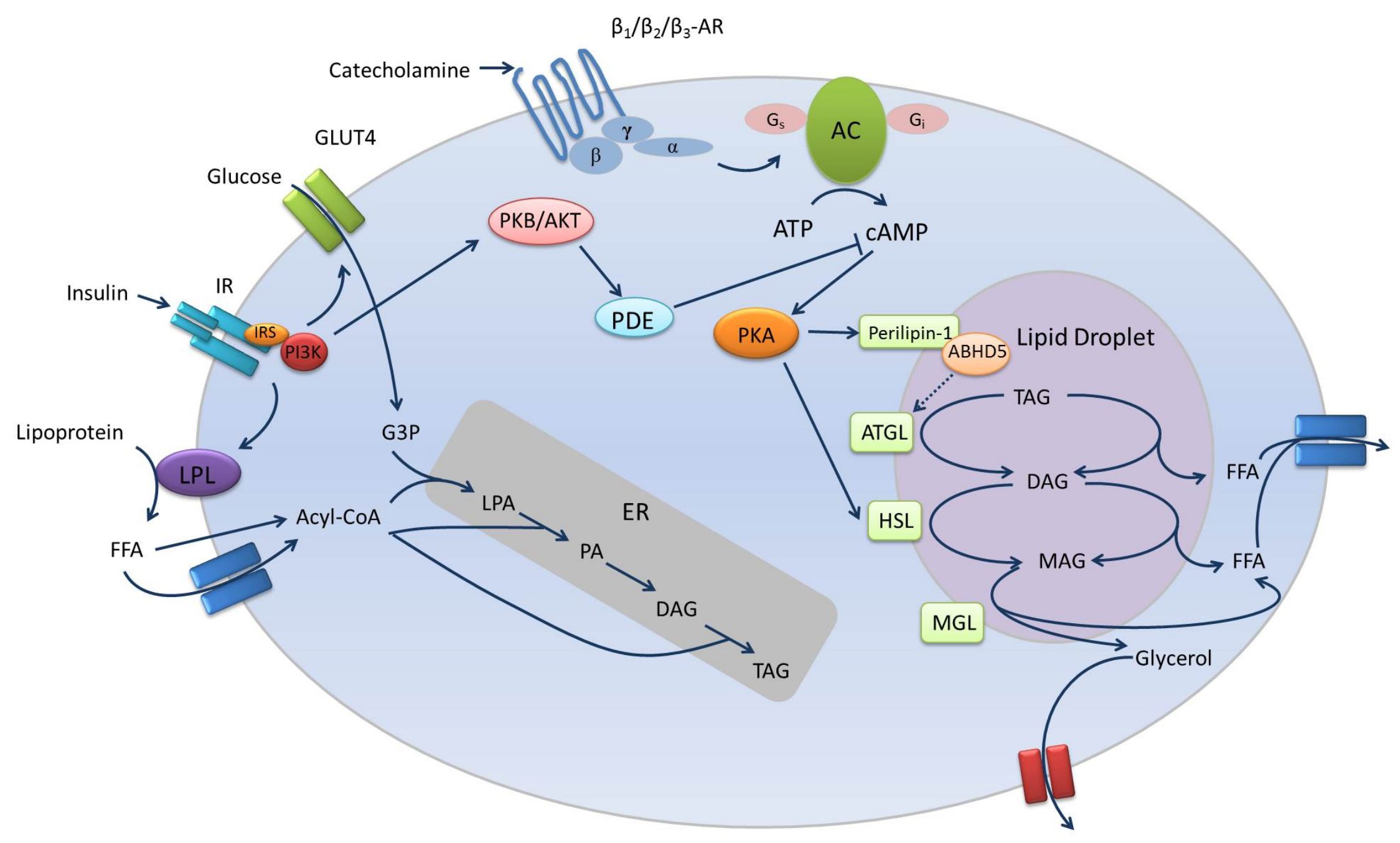


also exist in adult humans and are interspersed into the white adipose tissue (WAT) (Giralt and Villarroya, 2013). But these brown adipocytes are different from the classical brown adipocytes and share many similarities with the white adipocytes cell linage, and therefore, sometimes are referred to as the "inducible, beige, brite" adipocytes (Giralt and Villarroya, 2013). White adipocytes contain a single lipid droplet, while brown adipocytes contain multilocular droplets and higher density of mitochondria. BAT can be induced by cold or exercise (Cannon and Nedergaard, 2004), and primarily function to promote non-shivering thermogenesis. Inducible thermogenic genes in BAT genes include: uncoupling protein 1 (UCP1), PRD1-BF-1-RIZ1 homologous domain containing protein-16 (PRDM16), peroxisome proliferator-activated receptor gamma (PPAR $\gamma$ ) coactivator 1-alpha (PGC-1 $\alpha$ ), PGC-1 $\beta$, and CIDEA (Kajimura et al., 2010).

WAT represents the majority of body fat. WAT can be further classified by its location into subcutaneous adipose tissue which is underneath the skin and visceral (or abdominal) adipose tissue which is located inside the abdominal cavity. Mesenteric, epididymal white adipose tissue and perirenal depots all belong to visceral adipose tissue. Although subcutaneous and visceral adipose tissues are both WAT, they are distinct in terms of functional implications. For example, enlargement of visceral WAT is more associated with metabolic syndrome than that of subcutaneous WAT (Ibrahim, 2010). Visceral adipose tissue is also richer in blood and more innervated (Ibrahim, 2010). In addition, visceral adipocytes are more metabolically active, hormonally responsive to adrenergic-stimulated lipolysis than subcutaneous adipocytes (Arner, 1995; Arner et al., 1990; Hellmer et al., 1992).

\subsubsection{Hypertrophy and Hyperplasia of Adipose Tissue}

Adipose tissue expands in obesity to store excess energy. As excess energy accumulation within the body continues, adipocytes will expand in size (hypertrophy) and/or increase in number (hyperplasia) to store more lipids. Hyperplastic growth is found solely at early stages of adipose tissue development in human and the adipocyte number become stable in adulthood (Spalding et al., 2008). In rodents, both size and number of adipocyte can change along development of obesity, with the observation that hypertrophy occurs prior to hyperplasia (Faust et al., 1978). Although it seems feasible to combat obesity by restricting adipose tissue expansion, as hypertrophy and hyperplasia are known to facilitate obesity, modulating transcription factors to inhibit adipocyte development and adipose tissue expansion concomitantly affects metabolic homeostasis (Stephens, 2012).

The dominant cell type within adipose tissue is mature adipocytes. Nonetheless, endothelial cells, vascular smooth muscle cells, immune cells such as macrophages as well as fibroblasts can also be found in the adipose tissue. In addition, adipocyte precursor cells coexist within adipose tissue, which can be regenerated into mature adipocytes through a process called adipogenesis. 
Several immortalized cellular models can be used to study adipogenesis, such as the 3T3-L1 and 3T3-F442A cell lines derived from Swiss mouse embryos, embryonic stem cells, primary mouse embryonic fibroblasts (MEFs), immortalized MEFs, immortalized brown-adipose stromal cells, and immortalized multipotent precursor cells. Nonetheless, the methodologies used to differentiate these cells may be different. The 3T3-L1 and 3T3-F442A cell lines could be differentiated to mature adipocytes when treated with a hormonal cocktail, whereas the differentiation of immortalized MEFs requires the overexpression of a pro-adipogenic transcription factor such as PPAR $\gamma$ or CCAAT-enhancer- binding protein alpha (C/EBP $\alpha$ ) (Rosen and MacDougald, 2006).

One of the major adipogenic regulators is the transcription factor PPAR $\gamma$, which is both necessary and sufficient for adipogenesis (Rosen et al., 2000). Other transcription factors that facilitate adipogenesis include members of the C/EBP family, members of the Krüppel-like factors (KLFs) family of proteins, STATs, and SREBP-1 (Sarjeant and Stephens, 2012). However, PPAR $\gamma$ expression and/or activity are likely required for the action of most of the pro-adipogenic factors (Rosen and MacDougald, 2006). Negative transcription factors for adipogenesis include the surface protein preadipocyte factor1 (PREF1) and members of the GATA family, which function in part by repressing PPAR $\gamma$ expression (Tong et al., 2000). These pro- and anti- adipogenic factors often work in complex with co-activators or co-repressors. The most well-studied anti-adipogenic nuclear receptor co-repressor is NcoR (Li et al., 2011). PPAR $\gamma$ has two protein isoforms that are generated by alternative splicing, i.e. PPAR $\gamma 1$ and PPAR $\gamma 2$. While these two proteins are induced during adipogenesis, their relative importance remains elusive. PPAR $\gamma 2$ is believed to be more efficient in promoting adipogenesis than the other isoform PPAR $\gamma 1$, but is not necessarily required for adipocyte development in vivo (Mueller et al., 2002; Zhang et al., 2004).

\subsection{NEDD4}

\subsubsection{E3 Ligases in Metabolism}

The conjugation of ubiquitin to a protein substrate is a three-step process. In the first step, the C-terminus of ubiquitin binds a Cys residue within a ubiquitin-activating enzyme (E1) by forming a thioester bond. E1 enzyme activates the ubiquitin and transfers it to a specific Cys residue within a ubiquitin-conjugating enzymes (E2s). E2s can then transfer ubiquitin directly to substrates when working with RING (Really Interesting New Gene) domain ubiquitin-protein ligases (E3s), or through specific HECT E3s. Nterminus of ubiquitin is thus linked to specific internal Lys residues of target proteins. Substrates can undergo monoubiquitylation, multi-ubiquitylation or polyubiquitylation. Polyubiquitylated proteins are normally degraded by the $26 \mathrm{~S}$ proteasome (Rotin and Kumar, 2009). Besides its most notable proteolytic function, ubiquitination also regulates receptor internalization, protein lysosomal sorting, interaction, subcellular distribution, and gene transcription, etc. (Chen and Sun, 2009). 
In recent years, several E3 ligases have been reported to be implicated in the regulation of body metabolism. For example, the Casitas B-lineage lymphoma (Cbl) homologue, Cbl-b, which belongs to the RING-type E3 ligase has been reported to regulate obesity and macrophage activation. In a report by Hirasaka et al., $C b l-b^{-/-}$mice displayed enhanced insulin resistance and macrophage activation in adipose tissue (Hirasaka et al., 2007). A similar conclusion was reached in Abe et al.'s study: Cbl-b deficiency enhanced saturated FFA-induced macrophage activation and exaggerated HFD-associated insulin resistance (Abe et al., 2013). Depletion of another Cbl homologue, c-Cbl, reduced adiposity, improved insulin sensitivity (Molero et al., 2004) and protected mice against HFD-induced obesity (Molero et al., 2006). Other examples are the muscle-specific mitsugumin 53 (MG53, also known as TRIM72) and cullin-7 (CUL7), two RING-type E3 ligases which mediated IRS-1 ubiquitination and degradation, and therefore, negatively modulated insulin signaling (Lee et al., 2010; Xu et al., 2008; Yi et al., 2013). Loss and overexpression of MG53, protected against and triggered insulin resistance and metabolic syndrome in mice under HFD challenge (Song et al., 2013). Depletion of ITCH, a HECT-type E3 ligase involved in T-cell differentiation and inflammatory regulation, reversed HFD-induced obesity, the associated insulin resistance and liver steatosis (Marino et al., 2014). The beneficial effects of ITCH loss of function were likely due to the accelerated metabolism and increased differentiation of M2 macrophages, as well as the enhanced fatty acid oxidation in Itch-deficient mice (Marino et al., 2014). Consistently, in human adipose tissue, expression of ITCH was negatively correlated with that of the M2 macrophage markers (Marino et al., 2014).

\subsubsection{NEDD4 and Its Substrates}

Neural precursor cell expressed, developmentally down-regulated 4 (NEDD4) is a HECT-type E3 ubiquitin ligase, which was first identified from a screen for developmentally regulated gene in early embryonic mouse central nervous system (CNS) in 1997 (Kumar et al., 1997). NEDD4 is believed to be the ancestral member of the 9membered NEDD4 family (Yang and Kumar, 2010). Mouse and rat NEDD4 are almost identical with only two amino acids difference. However, human NEDD4 has 5 splice variants ranging from 900 to 1302 amino acid in length. NEDD4 contains an N-terminal $\mathrm{C} 2$ domain for calcium/lipid and/or protein binding, 3 to $4 \mathrm{WW}$ domains for proteinprotein interaction, and a C-terminal catalytic ligase domain (HECT domain). WW domains is the main component determining the substrates binding by NEDD4 via binding PPxY (PY) or LPSY motifs, although the C2 domain of NEDD4 also bind substrates (Yang and Kumar, 2010).

Despite the fact that many studies support NEDD4 as a critical player in early embryonic development (Cao et al., 2008; Fouladkou et al., 2010; Liu et al., 2009), knowledge toward the patho-physiological functions of this protein in postnatal life is limited. Recent experimental results indicate that NEDD4 plays a pivotal role in regulating receptor signaling by regulating receptor endocytosis (Rotin et al., 2000), A partial list of substrates of NEDD4 includes: epidermal growth factor receptor (EGFR) 
(Lin et al., 2010), vascular endothelial growth factor receptor 2 (VEGF-R2) (Murdaca et al., 2004), insulin-like growth factor receptor type 1 (IGF-1R) (Vecchione et al., 2003), IR (Ramos et al., 2006), Notch1 (Koncarevic et al., 2007), PTEN (Wang et al., 2007), beta-2 adrenergic receptor $\left(\beta_{2}-\mathrm{AR}\right)$ (Nabhan et al., 2010; Shenoy et al., 2008), fibroblast growth factor receptor (FGFR1) (Persaud et al., 2011), AKT (Fan et al., 2013), and Ras (Zeng et al., 2014). Since most of these substrates of NEDD4 are active players in various metabolic pathways, hence, we hypothesized that NEDD4 could be a new player in the regulation of metabolism.

1.3.2.1 IGR-1R and IR. In several cell-based studies, NEDD4 was found in complex with growth factor bound-receptor 10 (Grb10) to mediate ligand-stimulated IR and IGF-1R ubiquitination, which subsequently facilitated their internalization and/or degradation (Monami et al., 2008; Ramos et al., 2006; Vecchione et al., 2003). Reduction in NEDD4 expression was presumed to stabilize IR and IGF-1R upon ligand stimulation, and thus potentiats insulin and IGF-1 signaling. However, more recent evidence from in vivo studies revealed that insulin/IGF-1 signaling in NEDD4 knockout embryos were reduced, suggesting a more complex role of NEDD4 in vivo. Mice homozygous for targeted mutation of the Nedd4 gene died during or shortly after birth and exhibited severe intrauterine growth retardation (body weight of $\mathrm{Ne} d d 4^{--}$embryos and newborn pups were only $35 \%$ of normal) (Cao et al., 2008; Liu et al., 2009). The growth deficiency in Nedd4-haploinsufficient mice are similar in severity to the previously characterized mice carrying an Igflr null mutation (45\% of normal birth weight) (Baker et al., 1993; Liu et al., 1993), suggesting an impaired IGF-1 signaling in the absence of NEDD4 during embryogenesis. Indeed, in cultured Nedd4 $4^{-/}$MEFs, cell surface expression of both IGF-IR and IR were found to be substantially reduced (Cao et al., 2008). Additionally, the authors speculated that reduction in insulin and IGF-1 signaling by NEDD4 depletion is likely through increased expression of Grb10 (Cao et al., 2008), a known negative regulator for IR and IGF-1R function. The level of Grb10 was elevated in NEDD4 knockout MEFs, which may in turn impede insulin/IGF-1 signaling. The lethality of NEDD4 knockout mice could be partially rescued by crossbreeding with Grb10-deficient mice (Cao et al., 2008). Since Grb10 also binds IR (Liu and Roth, 1995) which shares structural and downstream signaling similarities with IGF-1R, it is possible that NEDD4 also regulates insulin signaling via elevated Grb10; Grb10 associates preferentially with IR as compared with IGF-1R (Laviola et al., 1997).

1.3.2.2 $\boldsymbol{\beta}_{2}$-AR. Agonist treatment for different length of time resulted in different effects on $\beta_{2}$-AR trafficking. Short exposure within $1 \mathrm{hr}$ caused internalization of the phosphorylated receptor which were recycled to the membrane after dephosphorylation; prolonged exposure from hours to days caused receptor protein degradation (Shenoy et al., 2001). NEDD4 has been demonstrated to be an essential E3 ligase mediating agoniststimulated ubiquitination and degradation of $\beta_{2}$-AR (Nabhan et al., 2010; Shenoy et al., 2008). In Shenoy et al.'s study, expression of a dominant negative NEDD4 or siRNA targeting NEDD4 specifically inhibited isoproterenol-induced ubiquitination and degradation of the $\beta_{2}$-AR in HEK-293 cells, whereas other closely related E3 ligases had 
no effects (Shenoy et al., 2008). Moreover, the interaction between NEDD4 and $\beta_{2}$-AR was ablated by an siRNA targeting arrestin beta 2 ( $\beta$-arrestin2), suggesting an adaptor role for $\beta$-arrestin 2 in the interaction (Shenoy et al., 2008). In Nabhans et al.'s study, an alpha arrestin, arrestin domain-containing 3 (ARRDC3), was found to be involved in $\beta_{2}$ AR downregulation as revealed by a genome-wide RNA interference screen using a cell line that expressed a distally truncated form of $\beta_{2}$-AR ( $\beta_{2}$-ARt) (Nabhan et al., 2010), as the $\beta_{2}$-ARt underwent faster receptor degradation upon agonist stimulation than the wildtype receptor (Cao et al., 1999). Interestingly, they found that ARRDC3 also served as an adaptor protein that regulated $\beta_{2}$-AR via recruiting the E3 ligase NEDD4 (Nabhan et al., 2010) (Figure 1.2). In support of these in vitro observations, in vivo evidence collected by another group of researchers showed that the Arrdc3-deficient mice were resistant to age-induced obesity (Patwari et al., 2011). The Arrdc3-deficient mice exhibited enhanced energy expenditure with increased thermogenesis in both BAT and WAT, and enhanced cAMP accumulation and lipolysis in response to norepinephrine (Patwari et al., 2011), suggesting increased $\beta$-AR signaling in vivo when the interaction between NEDD4 and $\beta_{2}$-AR was abrogated.

\subsection{Summary}

The HECT-type E3 ubiquitin ligase NEDD4 has a large number of substrates that are active players in the growth factor receptor signaling, implying a potential role of NEDD4 itself in regulating whole-body metabolism. Besides the receptors mentioned previously, PTEN (Kwak et al., 2010; Wang et al., 2007), AKT (Fan et al., 2013), and Ras (Zeng et al., 2014) are also demonstrated to be the substrates of NEDD4 in different context. To investigate the function of NEDD4 in metabolic regulation in vivo, we characterized the metabolic phenotypes of Nedd4-haploinsufficient mice in a dietinduced obesity (DIO) model, due to the fact that homozygous Nedd4 deletion causes embryonic/perinatal lethality. In this study, we also tested the possible impact of NEDD4 reduction on cognitive function and anxiety behaviors, as NEDD4 is highly expressed in the brain and regulates several growth factor receptor signaling pathways involved in CNS development and function. 


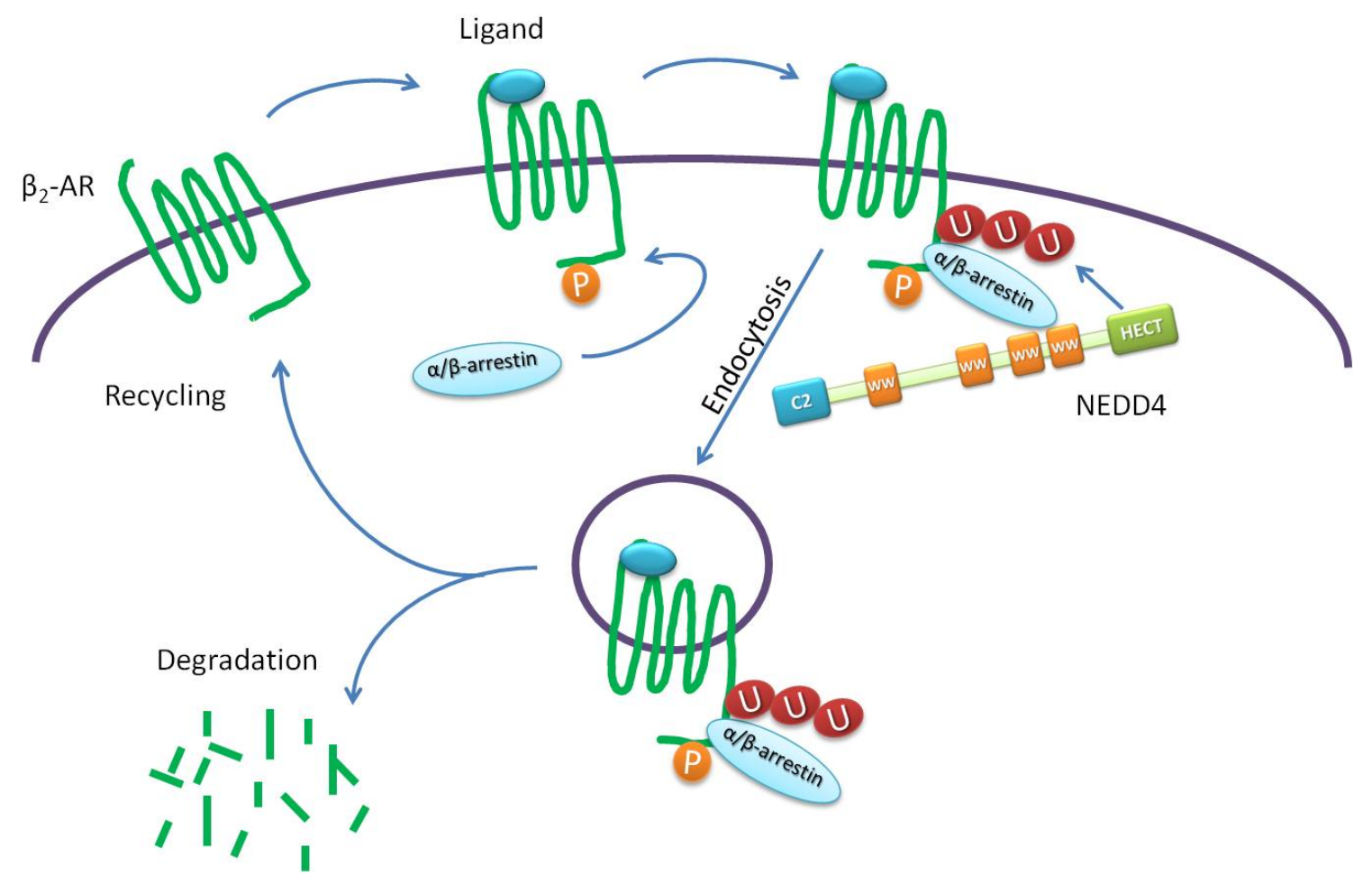

Figure 1.2 Regulation of $\beta_{2}-A R$ Endocytosis and Degradation by NEDD4.

Ligand binding induces phosphorylation within $\beta_{2}$-AR and the recruitment of $\alpha$ - or $\beta$ arrestins to the receptor. The $\alpha$ - or $\beta$-arrestins serve as the binding sites for the WW domains of NEDD4, therefore bringing NEDD4 in proximity to $\beta_{2}$-AR. The HECT domain within NEDD4 catalyzes ubiquitination of $\beta_{2}$-AR serving as a signal for receptor endocytosis and subsequent degradation. 


\section{CHAPTER 2. MATERIALS AND METHODS}

\subsection{Animal Studies}

The Nedd4-haploinsufficient mice (Nedd4 $\left.4^{G t(O S T 16211) L e x}\right)$ were originally generated by Lexicon Genetic Incorporated and are stocked at the Mutant Mouse Regional Resource Center (MMRRC) (http://www.mmrrc.org/strains/11742/011742.html). All mice were kept on a 12/12 light/dark cycle with lights on at 6:00 AM in a pathogen-free animal facility. The animals had ad libitum access to water and food at all times. Food was only removed when required.

\subsubsection{Genotyping}

Tail tissue $(2-5 \mathrm{~mm})$ from young $(<21$ days) mice was taken by a sterilized scissor. DNA was isolated by phenol-chloroform extraction. PCR was performed with GoTaq Flexi DNA Polymerase (Promega). Nedd4 mice were genotyped by PCR with the following primer sequences: Nedd4 wild type allele-forward GGA GTC TTT GGA TAT TGT AAG AGC; Nedd4 mutant allele-forward AAA TGG CGT TAC TTA AGC TG CTT GC; and a common reverse GAG CGT GCG CCT CAC AAG TAT GA.

\subsubsection{Food Intake, Body Weight, and Body Length Measurement}

Mice were fed either a normal control diet (8640, Teklad, Harlan Laboratories) with $17 \%$ of total calories from fat, $54 \%$ from carbohydrate and $29 \%$ from protein, or a HFD (TD.06414, Teklad, Harlan Laboratories) with $60.3 \%$ of total calories from fat, $21.3 \%$ from carbohydrate and $18.4 \%$ from protein, starting from 6 weeks of age. The HFD is packed in the form of pellet and kept at $4{ }^{\circ} \mathrm{C}$ until use. For food intake measurement, mice at 12-16 weeks of age were ad-libitum fed a known amount of food. After $24 \mathrm{hr}$, the remaining food pellets were weighed. The consumption of food (absolute food intake) was calculated by the difference. Animals' food intake was monitored on a daily basis for a continuous 7-day period. Body weights were monitored on a weekly basis with a small scale. For body length measurement, mice between 12-16 weeks of age were anesthetized or euthanized. Then a ruler was hold up against the body to measure the length from nose to anus.

\subsubsection{Glucose Tolerance Test (GTT) and Insulin Tolerance Test (ITT)}

For GTT, mice fasted overnight were weighed. Glucose were intraperitoneally (i.p.) injected to the mice at a dose of $1.5 \mathrm{~g} / \mathrm{kg}$ body weight. Blood glucose level was measured with a glucometer (OneTouch Ultra glucometer, LifeScan) in a drop of blood at time 0 (before the injection of glucose) and 30, 60, 90, 120, and 180 min after glucose injection. For ITT, mice were fasted for $4 \mathrm{hr}$ and then i.p. injected with insulin at 0.75 
$\mathrm{U} / \mathrm{kg}$ body weight. Blood glucose concentrations were read from a glucometer with a drop of blood at time 0 and 30, 60, and 90 min after insulin administration.

\subsubsection{Insulin Signaling}

For peripheral insulin signaling analysis, adult mice fasted overnight were i.p. injected with $5 \mathrm{U} / \mathrm{kg}$ of insulin diluted in normal saline or an equal volume of saline. Mice were euthanized 15 min after injection. Liver, gastrocnemius muscle, and epididymal WAT were dissected and immediately frozen in liquid nitrogen. For central insulin signaling analysis, P0 neonatal mice were euthanized by decapitation. The entire brains were carefully removed by opening the skull with a dissecting scissor. The isolated brains were placed in a clean tissue culture plate $(100 \mathrm{~mm})$ containing sterile Hank's Balanced Salt Solution (HBSS). Under a light microscope, the cerebellum was removed; then the thin and transparent layer which contains fibrous and vascular tissues surrounding the forebrain was gently removed by dissecting forceps. Tissues containing hypothalamus, amygdala, thalamus and striatum were carefully removed by dissecting forceps. Other non-cortical tissues were also carefully removed, leaving only a thin layer of cortical tissue. These cortical tissues were minced into pieces of relatively equal size with dissecting forceps and placed into different wells prefilled with $500 \mu \mathrm{l}$ of HBSS of a 24-well plate. These tissues were then incubated with insulin at different doses or for different time (up to $60 \mathrm{~min}$ ). Tissue lysates were subjected to Western-blot analysis.

\subsubsection{Measurement of Metabolites and Hormones}

Blood was collected through lateral saphenous vein. Briefly, the animal was secured in a restrainer. The rear leg was stretched out to its natural position. The hair was removed by a small shaver and swabbed using $70 \%$ ethanol. The vessel was punctured with a 25-26 gauge needle and blood was collected with a heparinized capillary tube. Blood glucose levels were measured using a glucometer as mentioned in GTT and ITT. After collection of blood, the blood was allowed to clot by leaving it undisturbed at room temperature (RT) for $30 \mathrm{~min}$. Serum was obtained by centrifuging clotted blood in tubes at $1,600 \mathrm{~g}$ for $10 \mathrm{~min}$ at $4^{\circ} \mathrm{C}$ and stored at $-80^{\circ} \mathrm{C}$ until use. Serum insulin levels were determined with murine ELISA kits (Crystal Chem). Total serum TGs, glycerol, cholesterol, and FFAs were assayed using enzymatic methods with a colorimetric or fluorometric assay kit (Cayman). Serum insulin levels during GTT were determined with a murine ELISA kits (Crystal Chem).

\subsubsection{Histology}

Mouse was cardiac-perfused first with phosphate buffered saline (PBS) to clear blood, then with 4\% paraformaldehyde (PFA) for fixation. Epididymal adipose tissue and liver was dissected and post-fixed in 4\% PFA for $24 \mathrm{hr}$. Adipose tissue was subjected to paraffin embedding and sectioning to $10 \mu \mathrm{m}$ slices. Liver was immersed in $30 \%$ sucrose 
prepared in PBS for dehydration, then embedded in optimum cutting temperature compound (OCT), and cryosectioned with a cryostat (Leica CM 1860).

Hematoxylin and eosin (H\&E) staining of sections was performed according to standard procedures (Fischer et al., 2008). Briefly, sections were stained with hematoxylin for nuclei, rinsed with running tap water, counterstained with eosin, and dehydrated before mounting. The paraffin embedded sections were dewaxed and rehydrated by sequentially immersing in xylene, ethanol, and water before H\&E staining. Microscopic pictures were taken and adipocyte size was measured in 300-400 cells per mouse in randomly selected fields using ImageJ software (National Institutes of Health, Bethesda, Maryland; http://imagej.nih.gov/ij/). For Oil red O staining in liver, cryosections were air dried and fixed in 10\% formalin. Sections were briefly washed with running tap water, immersed in $60 \%$ isopropanol for $5 \mathrm{~min}$, and stained with freshly prepared oil red $\mathrm{O}$ working solution for $15 \mathrm{~min}$. Then, sections were briefly counterstained for nuclei with hematoxylin, rinsed with distilled water and mounted with glycerine jelly.

\subsubsection{Lipolysis Assay}

During the in vivo lipolysis assay, the non-selective $\beta$-agonist isoproterenol was i.p. injected into mice fasted overnight, at $10 \mathrm{mg} / \mathrm{kg}$ body weight. Blood samples were collected at time 0 and 10, 30, and 60 min after the injection. The ex vivo lipolysis assay was performed as described in (Qiao et al., 2011). During primary adipocyte lipolysis assay (Viswanadha and Londos, 2006, 2008), minced epididymal WAT was digested in a Krebs-Ringer bicarbonate (KRB) buffer ( $\mathrm{pH} 7.4$ ) plus $3 \%$ fatty acid-free BSA fraction V, $0.5 \mathrm{mM}$ adenosine, and $1 \mathrm{mg} / \mathrm{mL}$ type I collagenase at $37^{\circ} \mathrm{C}$ for $45 \mathrm{~min}$. After digestion and three washes with KRB plus 3\% fatty acid-free BSA, the dissociated adipocytes were counted with a hemocytometer under a microscope. Approximately 12,000 adipocytes were resuspended in $400 \mu \mathrm{L} \mathrm{KRB}$ buffer plus 3\% fatty acid-free BSA. Isoproterenol was then added to the buffer at $1 \mu \mathrm{M}$. After $1 \mathrm{hr}$ incubation at $37^{\circ} \mathrm{C}$ with gentle shaking, samples were centrifuged and the infranatant (media below the floating adipocyte layers) was removed and stored at $-80^{\circ} \mathrm{C}$ before use. Glycerol levels were measured by using a colorimetric assay kit (Cayman) following the manual.

\subsubsection{Fear Conditioning}

Mouse was subjected to fear conditioning training on day 1, a context fear retrieval test and a tone fear retrieval test (at least $3 \mathrm{hr}$ apart) on day 2 . In the fear conditioning trial, mouse was gently placed in a chamber of $30 \times 30 \times 25 \mathrm{~cm}$ in dimension (Coulbourn Instruments). After a 3 min recording for baseline activity, two paired tone-shock stimuli were presented one minute apart. The paired tone-shock stimuli consisted of a $20 \mathrm{sec}$ tone $(2800 \mathrm{~Hz}, 82 \mathrm{~dB})$ and a $2 \mathrm{sec}$ electrical footshock $(0.7 \mathrm{~mA})$ at the distal end of the tone. One minute after the second tone-shock stimulus, mouse was returned to its home cage. During the context fear retrieval trial on day 2, mouse was 
placed back to the same chamber for a 3 min period. The freezing time of the mouse during the testing period was recorded. During the tone fear retrieval trial on day 2 , mouse was placed in the same box decorated with colored plastic boards. After a 3 min recording for baseline activity, a $20 \mathrm{sec}$ tone similar to day 1 was presented. The freezing time of the mouse during the testing period was recorded. All experiments were conducted between 9 AM and 5 PM.

\subsubsection{Cross Maze}

Mouse was placed in the entry arm of the cross-maze and allowed to explore freely for 5 min with a camera on top. At the end of the study, videos were replayed. The pattern of arm choices was recorded and the total number of entries was counted. An entry was counted when four limbs of the mouse are in the arm.

\subsubsection{Morris Water Maze Test}

At approximately 2 months of age, the spatial learning and memory in the young Nedd $4^{+/-}$mice and their littermates were evaluated by the Morris water maze test (Morris, 1984). During the water maze test, mouse was allowed to swim in a circular pool $(114 \mathrm{~cm}$ in diameter). Water was colored with a non-toxic paint to make opaque. A circular escape platform of $10 \mathrm{~cm}$ in diameter was placed under the surface of the water about $0.5 \mathrm{~cm}$ in depth, to allow the mouse to escape from swimming. Four trials per day were conducted during the visible and hidden platform training. In the visible platform training, the submerged escape platform was marked by a visible sign above the surface of the water. Mouse was released into the swimming pool at different positions during each of the four trials. The latency for the mouse to find the platform was recorded. Mouse that did not find the platform in $60 \mathrm{sec}$ was assisted to stay on the platform for an additional $10 \mathrm{sec}$. After the training section, mouse was tested a probe trial, in which the platform was removed from the pool and mouse was allowed to swim for $60 \mathrm{sec}$. The swimming track of mouse was recorded.

\subsubsection{Open Field Test}

Mouse was placed in an open-field box $(47 \times 37 \times 20 \mathrm{~cm}$, Accuscan Instruments $)$, equipped with 16 infrared beams that automatically measures total locomotor activity by beam-breaks and with a camera on top that measures the distance the mouse travelled. Mouse was gently placed in the box and allowed to explore freely for $10 \mathrm{~min}$. About $25 \%$ of the field $(47 \times 37 \times 20 \mathrm{~cm})$ was determined as center. Experiments were performed between 9 AM to $5 \mathrm{PM}$. 


\subsubsection{Light/Dark Exploration}

A plastic chamber $(27 \times 27 \mathrm{~cm}$ in dimension $)$ was used for light/dark exploration. The chamber was segregated into opaque and non-opaque compartments. Mouse was allowed to transit between the two compartments through a hole on the segregation wall. Mouse was placed in the light side and allowed to explore for a 5-min session. The movement of mouse was recorded by a camera on top. Several parameters (latency to enter the dark, latency to re-enter the light, total time spent in the dark compartment and the number of transitions between lighted and dark compartments) were analyzed at the end of the study.

\subsection{Biochemical Studies}

\subsubsection{Cells}

The 3T3-L1 preadipocytes were cultured in Dulbecco's Modified Eagle Medium (DMEM) supplemented with 10\% bovine calf serum. Cells were split 1:10 every 2 days to prevent reaching confluency. For adipocyte differentiation, the preadipocytes were allowed to grow to confluency in $10 \%$ bovine calf serum. Two days post confluency, cells were switched to DMEM supplemented with 10\% fetal bovine serum (FBS) and stimulated with a differentiation cocktail (IDX) containing $0.5 \mathrm{mM}$ isobutylmethylxanthine (IBMX), $1 \mu \mathrm{M}$ dexamethasone, and $1.5 \mu \mathrm{g} / \mathrm{mL}$ insulin. Three days after IDX induction, cells were fed with DMEM plus $10 \% \mathrm{FBS}$ and $1.5 \mu \mathrm{g} / \mathrm{mL}$ insulin. Three days after, cells were cultured in DMEM plus 10\% FBS and media was replaced every 2-3 days until full differentiation was achieved. The HEK293 cells were cultured in DMEM supplemented with 10\% FBS. The HEK293 cells stably expressed FLAG-tagged $\beta_{2}$-AR (a kind gift from Dr. Suleiman Bahouth at UTHSC) were maintained in DMEM plus 10\% FBS and $200 \mu \mathrm{g} / \mathrm{mL}$ of G418. Media was replaced every 2-3 days. All cells were incubated in $95 \% \mathrm{O}_{2}$ and $5 \% \mathrm{CO}_{2}$ at $37^{\circ} \mathrm{C}$.

\subsubsection{Adeno-associated Virus (AAV)-mediated Knockdown}

AAV-mediated knockdown of NEDD4 was performed by using the pAAV2.1.CMV.EGFP-U6.shRNA plasmid vector (a kind gift from Dr. Tonia Rex at Vanderbilt University) expressing short hairpin RNA (shRNA) targeting NEDD4 mRNA. Of three different shRNA constructs tested, the one showing most effective downregultion of NEDD4 protein and Nedd4 mRNA ( 50\%) was chosen for subsequent studies. The targeting sequence is TGG CGA TTT GTG AAC CGT A. Virus production was performed by the Gene Transfer Vector Core at the University of Iowa. 


\subsubsection{Western-blot Analysis}

Cells or tissues were lysed with radioimmunoprecipitation (RIPA) buffer supplemented with protease and phosphatase inhibitors. Cell debris in lysates was removed by centrifugation at maximum speed at $4{ }^{\circ} \mathrm{C}$. Protein concentrations were determined by the bicinchoninic acid (BCA) method (Pierce). Five to $40 \mu \mathrm{g}$ of protein of each sample was electrophoresed on a Novex 4-20\% Tris-Glycine gel (Invitrogen). After transferring the proteins to PVDF membrane, the membrane was blocked at RT in $1 \times$ Tris Buffered Saline plus $0.05 \%$ Tween-20 (TBST) containing 5\% non-fat dry milk for 1 hr. After blocking, membrane was incubated overnight at $4^{\circ} \mathrm{C}$ in primary antibody diluted in $\mathrm{TBST} / 5 \% \mathrm{BSA} / 0.02 \% \mathrm{NaN}_{3}$. On the second day, the membrane was washed thoroughly with TBST and incubated in secondary horseradish peroxidase (HRP) linked antibody (GE Healthcare Life Sciences). Blot was washed three times with TBST followed by incubation with enhanced chemiluminescence (ECL) for signal development.

\subsubsection{Co-immunoprecipitation (Co-IP)}

Cells were lysed in a co-IP buffer (25 mM Tris-HCl, $150 \mathrm{mM} \mathrm{NaCl}$, and 1\% NP40) supplemented with protease and phosphatase inhibitors. After determining protein concentrations, 300-1,000 $\mu \mathrm{g}$ of total protein was resuspended in co-IP buffer. Then, 1-3 $\mu \mathrm{g}$ of antibody and $30 \mu \mathrm{l}$ of protein $\mathrm{A} / \mathrm{G}$ beads (Santa Cruz) were added to each sample for overnight incubation with gentle shaking at $4^{\circ} \mathrm{C}$. On the second day, beads were centrifuged at 8,000 rpm for $2 \mathrm{~min}$, washed three times with the co-IP buffer, and finally resuspended in $1 \times$ SDS sample buffer and denatured in boiling water for $5 \mathrm{~min}$. Samples were centrifuged again at maximum speed and the resulting supernatant was loaded directly on a Tris-Glycine gel or stored at $-80^{\circ} \mathrm{C}$ until use.

\subsubsection{RNA Isolation, cDNA Synthesis, and qRT-PCR}

Total RNA was isolated using Trizol reagent (Invitrogen). For the 3T3-L1 adipocyte samples, $500 \mu \mathrm{l}$ Trizol reagent was applied per well in a 12-well plate; for adipose tissue samples, $1 \mathrm{ml}$ Trizol reagent was applied per 50-100 mg of tissue. Cells were lysed by scraping or using a homogenizer. A proportional volume of chloroform was added to the samples $(0.2 \mathrm{ml}$ per $1 \mathrm{ml}$ of Trizol reagent used) and mixed thoroughly by shaking for $15 \mathrm{sec}$. The homogenates were allowed to sit at RT for 2-3 min, and subjected to centrifugation at $12,000 \times \mathrm{g}$ for $15 \mathrm{~min}$. The following steps were performed as described previously (Li et al., 2014). Primers used are listed in Table 2.1.

\subsubsection{Crude Membrane Fraction}

For crude membrane fraction from primary adipocytes, epididymal WAT was minced and digested in KRB buffer ( $\mathrm{pH} 7.4$ ) supplemented with $3 \%$ fatty acid-free BSA fraction $\mathrm{V}, 0.5 \mathrm{mM}$ adenosine, and $1 \mathrm{mg} / \mathrm{mL}$ type I collagenase at $37^{\circ} \mathrm{C}$ for $45 \mathrm{~min}$. After 
Table 2.1 Primers Used for qRT-PCR.

\begin{tabular}{lll}
\hline Name & \multicolumn{1}{c}{ Forward Primer Sequences } & Reverse Primer Sequences \\
\hline Leptin & caggatcaatgacatttcacaca & gctggtgaggacctgttgat \\
MIP-1 $\alpha$ & atgaaggtctccaccactgc & cccaggtctcttggagtca \\
MCP-1 & catccacgtgttggctca & gatcatcttgctggtgaatgagt \\
F4/80 & cctggacgaatcctgtgaa & tcccagagtgttgatgcaaa \\
TNF $\alpha$ & ccctcacactcagatcatcttct & gctacgacgtgggctacag \\
IL1 $\beta$ & agcttcaggcaggcagtatc & gtcacagaggatgggctctt \\
IL4 & ggtctcaacccccagctagt & gccgatgatctctctcaagtgat \\
IL10 & ctggacaacatactgctaaccg & gggcatcacttctaccaggtaa \\
UCP1 & ggcctctacgactcagtcca & taagccggctgagatcttgt \\
UCP2 & tttgggttggttgttttga & ggaggcagatgcaggtagat \\
UCP3 & tacccaaccttggctagacg & gtccgaggagagagcttgc \\
PGC-1 $\alpha$ & gaaagggccaaacagagaga & gtaaatcacacggcgctctt \\
PGC-1 $\beta$ & ctccagttccggctcctc & ccctctgctctcacgtctg \\
CIDEA & ttcaaggccgtttaagga & cctttggtgctaggcttgg \\
PRDM16 & cctaaggtgtgccagca & caccttccgcttttctaccc \\
NEDD4 & acgtgctgttcactgctgat & tcacaactcgtgtgtcatcg \\
aP2 & ggatggaaagtcgaccacaa & tggaagtcacgcctttcata \\
C/EBP $\alpha$ & gagcaaaaatgtgccttgatatt & tgcacccttcattttctcac \\
PPAR $\gamma$ & gaaagacaacggacaaatcacc & gggggtgatatgttgaacttg \\
\hline
\end{tabular}


digestion and three washes with $\mathrm{KRB}$ plus 3\% fatty acid-free BSA, the dissociated adipocytes were resuspended in one volume of STE buffer $(10 \mathrm{mM}$ Tris- $\mathrm{HCl} \mathrm{pH} 7.5,1$ $\mathrm{mM}$ EDTA pH 8.0, $250 \mathrm{mM}$ sucrose) prewarmed to $37^{\circ} \mathrm{C}$ supplemented with protease and phosphatase inhibitors. The adipocytes were homogenized at RT by six strokes of a large-bore Potter-Elvehjem homogenizer (Arthur H. Thomas Co.) fitted with a serratededge Teflon pestle. The homogenizer was driven by an Eberbach Con-torque motor operating at maximum speed. The homogenate was then transferred to a round bottom tube and spun at $50,000 \times \mathrm{g}$ for $30 \mathrm{~min}$ at $4^{\circ} \mathrm{C}$. The pellet which contains the crude fat cell membrane was resuspended in 10 volumes of $50 \mathrm{mM}$ Tris- $\mathrm{HCl}, 10 \mathrm{mM} \mathrm{MgCl}$, $\mathrm{pH}$ 8.0 containing protease and phosphatase inhibitors at $4^{\circ} \mathrm{C}$ and homogenized for 10 strokes as mentioned above. The homogenate was spun at $15,000 \times g$ for 15 min at $4^{\circ} \mathrm{C}$. The pellet was resuspended in RIPA buffer on ice for at least $1 \mathrm{hr}$. The lysate was spun again at maximum speed. The supernatant which contains the membrane proteins was transferred to a new tube for subsequent analysis.

For crude membrane fraction from HEK293 cells, cells were homogenated in one volume of STE buffer plus protein and phosphatase inhibitors by passing through a 25 gauge needle. The homogenates were transferred to a round bottom tubes and spun at $50,000 \times g$ for $30 \mathrm{~min}$ at $4^{\circ} \mathrm{C}$. The pellet was washed once in STE buffer containing protease and phosphatase inhibitors and spun again at $50,000 \times g$ for $30 \mathrm{~min}$ at $4^{\circ} \mathrm{C}$. The pellet was dissolved in RIPA buffer on ice for at least $1 \mathrm{hr}$. The lysate was spun again at maximum speed. The supernatant which contains the membrane proteins was transferred to a new tube for subsequent analysis.

\subsubsection{Oil Red O Staining in Differentiated 3T3-L1 Cells}

A stock Oil Red O solution was prepared by weighing out $150 \mathrm{mg}$ of Oil Red O powder and adding this to $50 \mathrm{~mL}$ of isopropanol. Then, 3 parts of Oil Red O stock solution with 2 parts distilled water were mixed and incubated for $10 \mathrm{~min}$ at RT. The Oil Red $\mathrm{O}$ working solution was filtered through Whatman filter paper. For Oil red $\mathrm{O}$ staining of differentiated 3T3-L1 adipocytes, cells cultured in a $35 \mathrm{~mm}$ dish were first fixed in 10\% formalin for 30-60 min. Cells were then briefly rinsed with 3 times with distilled water and incubated with $60 \%$ isopropanol for $5 \mathrm{~min}$. Freshly prepared Oil red O working solution were added to the cells for 5 min. Cultures were rinsed with RT tap water until the water rinses were clear.

\subsubsection{Dual Luciferase Reporter Assay}

HEK293 cells of relatively equal numbers were plated on 24-well plates. On the second day, seeded cells were transiently cotransfected with $250 \mathrm{ng} 3 \times$ PPRE-Luc, 250 ng PPAR 2 2-Flag, $10 \mathrm{ng}$ Renilla luciferase control reporter vector pRL-SV40 and $250 \mathrm{ng}$ of other plasmids using Lipofectamine 2000 transfection reagent (Invitrogen). At $32 \mathrm{hr}$ after the transfection, cells were treated with or without Rosiglitazone $(10 \mu \mathrm{M})$ for $16 \mathrm{hr}$. At $48 \mathrm{hr}$ after the transfection, luciferase assay was performed by a dual luciferase assay 
system (Promega) according to the manufacturer's instructions. The luciferase activity was adjusted by the Renilla luciferase activity to remove the variation caused by transfection efficiency. As NEDD4 overexpression or knockdown did not affect the survival of HEK293 cells, we did not normalize the luciferase activity to protein content.

\subsection{Materials}

\subsubsection{Antibodies}

Anti-NEDD4 was purchased from Millipore; anti- $\beta_{1}-A R(V-19)$, anti- $\beta_{2}-A R(H-$ 20), anti-CEBP/ $\alpha$ (D-5), and anti-PPAR $\gamma$ (E8) mouse monoclonal antibodies were purchased from Santa Cruz; another anti-PPAR $\gamma$ rabbit polyclonal antibody was from Thermo Scientific; anti-phospho-IGF-1R $\beta$ (Tyr1 135/1136)/IR $\beta$ (Tyr1150/1151) (19H7), anti-IR $\beta$, anti-IGF-1R $\beta$, anti-AKT, anti-AKT (Thr308), anti-AKT (Ser473), anti-STAT3, anti-STAT3 (Tyr705), anti-mTOR, anti-mTOR (Ser2448), anti-AMPK, and anti-AMPK (Thr172) antibodies were from Cell Signaling Technology.

\subsubsection{Reagents}

Hematoxylin, eosin, Oil Red O, insulin from bovine pancreas, (-)-isoproterenol hydrochloride, rosiglitazone, IBMX, dexamethasone, MG-132, cycloheximide (CHX), and chloroquine were purchased from Sigma-Aldrich; recombinant mouse leptin from E. Coli was from ProSpec; ICI 118,551 hydrochloride, CGP-20712 dihydrochloride, xamoterol hemifumarate, and salmeterol xinafoate were from Toris Bioscience.

\subsubsection{Plasmids}

The pcDNA3-PPAR $\gamma 2-F l a g$ was obtained from Addgene; pRc-CMV-T7-NEDD41-WT and pRc-CMV-T7-NEDD4-1-CS were kind gifts from Dr. Daniela Rotin at The Hospital for Sick Children in Canada; pSUPER-shNEDD4 was generated by our laboratory (Kwak et al., 2010); PPRE $\times 3$-TK-luc plasmid containing three copies of the PPAR response element (PPRE) upstream of a luciferase reporter was a generously provided by Dr. Edwards Park at UTHSC.

\subsection{Statistics}

Data were analyzed by GraphPad Prism 5.0. Two-tailed Student's $t$-test was used for statistical comparison between two groups; two-way ANOVA followed by bonferroni post-test correction was used for statistical comparison between groups when there were two factors. 


\section{CHAPTER 3. METABOLIC PHENOTYPE STUDIES}

\subsection{Introduction}

Obesity results from loss of homeostasis between lipid, protein, and carbohydrate metabolism. The delicate balance between protein synthesis and protein breakdown is crucial to keep our internal homeostasis under tight control. Protein degradation, or proteolysis is one of the strategies employed by the cell to remove abnormal proteins and prevent their aggregation, or to irreversibly inactivate proteins that are no longer needed (Ciechanover, 2005). The amino acids generated by protein degradation may then be reused for protein synthesis.

Besides the cell's autophagy-lysosome proteolysis, the ubiquitin-proteasome proteolysis represents a major protein degradation pathway. Defects in ubiquitindependent proteolysis have been associated with a number of human diseases, including metabolic disorders (Sakamoto, 2002). Although there are very few studies published so far, the importance of ubiquitin-dependent proteolysis in metabolic regulation is increasingly emphasized. The ubiquitin-proteasome system (UPS) requires the sequential and cooperative actions of E1, E2, and E3 enzymes. While E1 and E2 enzymes process ubiquitin, the E3 ligases are the main enzymes responsible for substrate specificity (Scheffner et al., 1995). Several members of the E3 ubiquitin ligase family have emerged as regulators of metabolism, as evident in the knockout mice for mitsugumin 53 (Song et al., 2013), ITCH (Marino et al., 2014), and two members of the Casitas B-lineage lymphoma (Cbl) family-Cbl-b and c-Cbl (Abe et al., 2013; Hirasaka et al., 2007; Molero et al., 2004; Molero et al., 2006). The involvement of these E3 ligases in HFDIO suggests important roles for E3 ligases in metabolic regulation.

Rsp5p, the most well-studied yeast E3 ligase, participates in important cellular processes including lipid and membrane synthesis (Hoppe et al., 2000). Its mammalian homologues - NEDD4 and ITCH - are two structurally related E3 ligases belonging to the nine-member HECT NEDD4 family. NEDD4 and ITCH both recognize proline-rich consensus sequence motifs (e.g., PPxY and PPLP), and thereby ubiquitinate several overlapping targets in vitro (Bray, 2006), including the aforementioned Cbl proteins (Magnifico et al., 2003). Comparison study of immunity using genetically modified mice indicates that they regulate distinct pathways in vivo (Yang et al., 2008). Comparing and distinguishing NEDD4 and ITCH regulated pathways in vivo are of particular importance to validate them as therapeutic targets. Recently, depletion of ITCH in mice was found to reverse HFD-induced obesity and its related metabolic disorders (Marino et al., 2014). Since NEDD4 knockout mice are embryonic/perinatal lethal, we created a HFDIO model in Nedd4-haploinsufficient mice. 


\subsection{Results}

\subsubsection{Genotype Verification of Nedd4-haploinsufficient Mice}

The Nedd4-haploinsufficient (Nedd $4^{+/}$) mice we used in this study were originally generated by Lexicon Genetic Incorporated. A gene-trapping vector insertion strategy was employed to disrupt the third WW domain between exon 17 and exon 18 (Figure 3.1A). The gene trap targeted $\mathrm{Nedd} 4^{+/-}$mice were generated on a $129 / \mathrm{SvEvBrd} \times$ C57BL6/J mix genetic background. A breeding pair (a male $\mathrm{Nedd}^{+/-}$and a female wild type) together with a female $\mathrm{Nedd} 4^{+/-}$were transferred from Dr. Weichun Lin's laboratory (University of Texas Southwestern Medical Center). The Nedd $4^{+/}$mice were viable, fertile, and devoid of gross phenotypic defects. However, a null mutation of

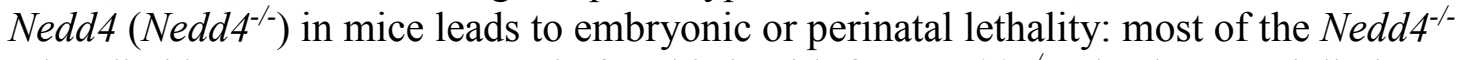
mice died between E15.5-E18.5 before birth with few Nedd4 $4^{-/}$mice born and died on P0 (Liu et al., 2009). Based on my observation, most of the Nedd $4^{+/}$- mice could live for at least 18 months.

We performed polymerase chain reaction (PCR) genotyping for $\mathrm{Nedd}^{+/-}$mice and western blot analysis for Nedd $4^{+/-}$and $\mathrm{Nedd}^{-/-} \mathrm{P} 0$ neonatal mice, confirming the presence of gene trap vector in Nedd $4^{+/-}$mice; reduction and absence of NEDD4 protein in Nedd $4^{+/-}$and $\mathrm{Ned}_{d} 4^{-/} \mathrm{P} 0$ neonatal mice, respectively (Figure 3.1B and C). To characterize the extent of NEDD4 protein reduction in Nedd $4^{+/}$mice in different organs, two pairs of Nedd $4^{+/-}$mice and their wild-type littermates were euthanized. As expected, NEDD4 protein levels were reduced at least 50\% in all organs tested (Figure 3.1D).

\subsubsection{Nedd4-haploinsufficient Mice Resist HFD-induced Obesity}

To elucidate the physiological role of NEDD4 and to examine the phenotype of $\mathrm{Nedd} 4^{+/-}$mice, we placed a cohort of 6-week old Nedd $4^{+-}$and wild-type mice on either a ND ( $17 \%$ calories from fat) or a HFD (60\% calories from fat), and monitored their body weights until age 9 months. When fed a ND, Nedd $4^{+/}$mice were lighter than their wildtype control mice from 6 weeks of age to until at least 9 months old in both genders (3.66 $\pm 0.05 \mathrm{~g}$ in male, $1.90 \pm 0.03 \mathrm{~g}$ in female) (Figure 3.2A). Their weight gain from 6 weeks to 9 months old were comparable between Nedd $4^{+-}$and wild-type mice (Figure 3.2B), suggesting that they grow in similar speed. The Nedd $4^{+/}$mice had reduced body mass at birth, and this difference in body mass was carried over into adulthood.

Surprisingly, in response to a HFD, there was a profound difference in the increase of body weight between Nedd $4^{+/}$and wild-type mice in both males and females (Figure 3.2A). After 30 weeks of HFD feeding, the total body weight gain in Nedd4 $4^{+-}$ mice decreased around $30 \%(P<0.001)$. At the end of the feeding, mice on ND had

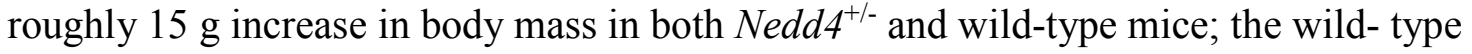
mice on HFD had an average of $43.2 \pm 1.0 \mathrm{~g}$ increase in body weight, while there was 
A

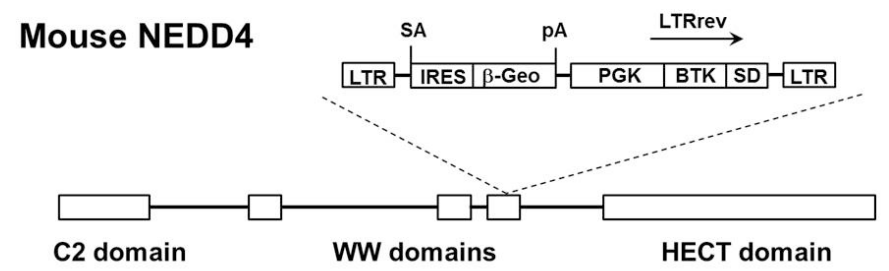

B

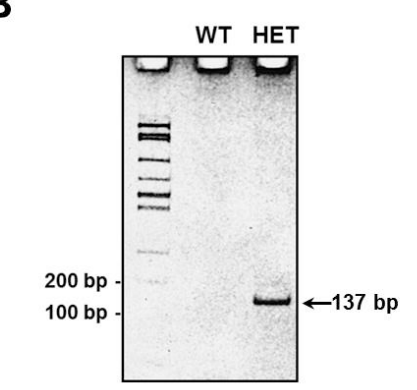

C

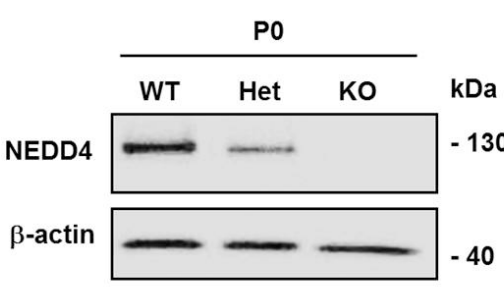

D

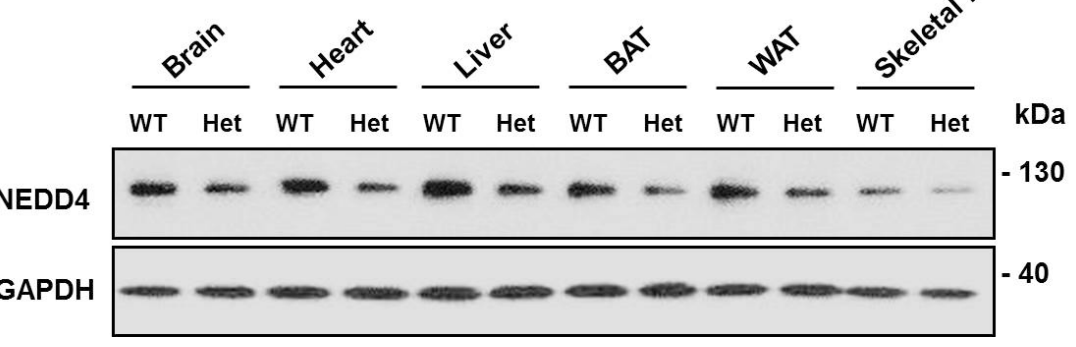

Figure 3.1 PCR and Western-blot Confirmation of Genotypes.

(A) A gene-trapping vector was inserted to the third WW domain of Nedd4 gene, disrupting between exon 17 and exon 18. (B) Representative image of PCR genotyping of wild-type (WT) and $\mathrm{Nedd}^{+/-}$(Het) mice. Amplified DNA fragments were visualized on an agarose gel stained with ethidium bromide. The 137-bp amplicon represented the mutant allele. (C) Analysis of whole-body tissue lysates from P0 neonatal mice by Western blot using an antibody against NEDD4. (D) Western blot analysis was performed in protein lysates from various organs, from two 2-month-old males. 
A
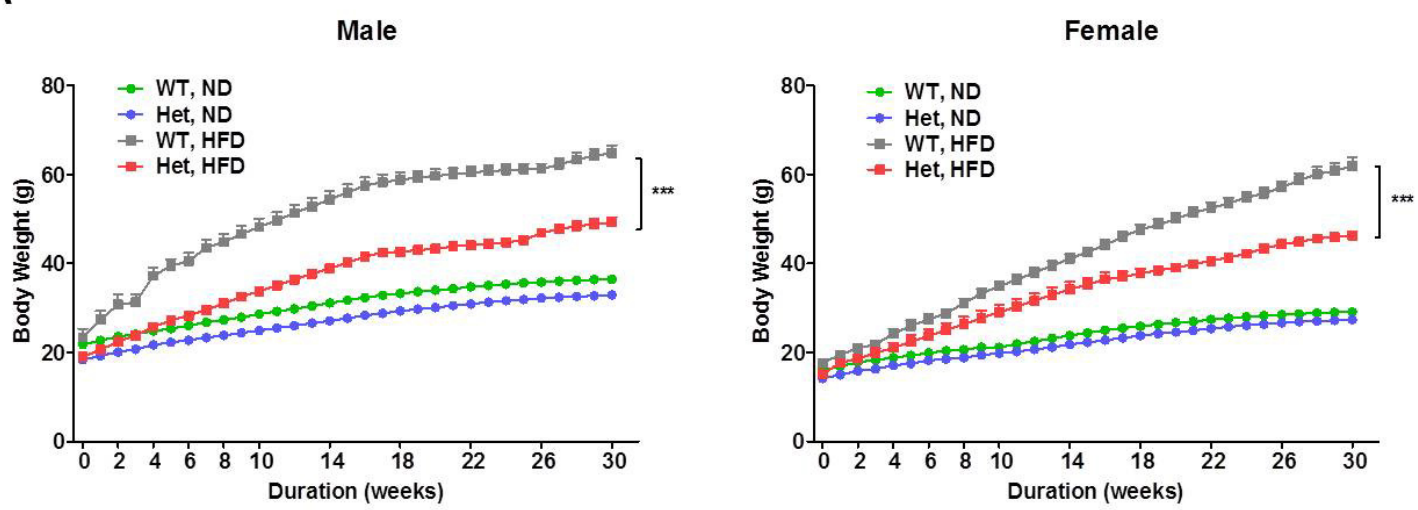

B

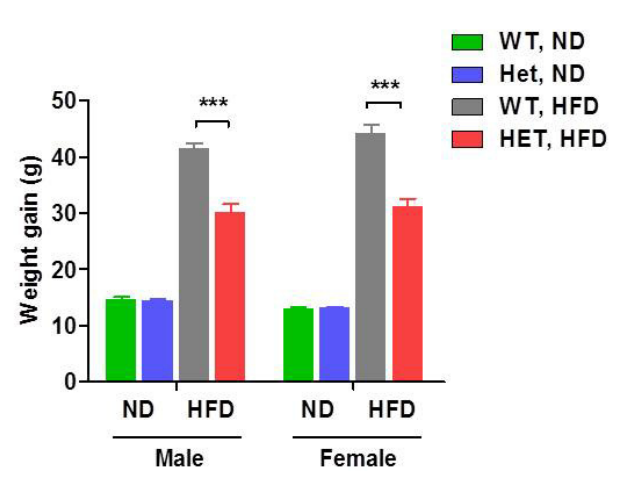

C

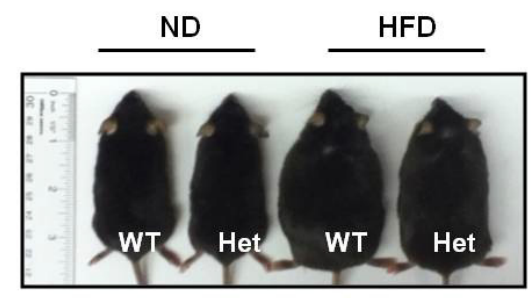

Figure 3.2 Nedd4-haploinsufficient Mice on HFD Exhibit Less Weight Gain.

(A) Body weight curves of littermate mice fed a normal diet (ND) or a high-fat diet (HFD) in males and females ( $\mathrm{n}=8-11$ per group). Mice at 6-week of age were started to be fed a HFD for 30 weeks. Body weights were monitored on a weekly basis. (B) Average weight gain after 30 weeks of diet feeding ( $n=8-11$ per group). (C) Representative photos of wild-type (WT) and Nedd $4^{+/-}$(Het) male mice fed a ND or a HFD at 22 weeks of age. All data are mean \pm SEM; $* * * P<0.001$. 
$30.6 \pm 1.0 \mathrm{~g}$ weight gain, $12 \mathrm{~g}$ less than the wild-type controls, in $\mathrm{Nedd4^{+/ }}$ mice on HFD (Figure 3.2B). Representative picture of the mice were shown in Figure 3.2C.

Nonetheless, $\mathrm{Nedd} 4^{+/-}$mice had unaltered physical activity, thermogenesis-related gene expression in BAT, and consumed calories proportional to their body weights. Therefore, activity and metabolism cannot explain the difference in HFD-induced weight gain between $\mathrm{Nedd}^{+/-}$and control mice (Figure 3.3).

To determine if the reduced weight gain was due to decreased body length, we measured the length of mice from anus to nose when the mice were anesthetized or euthanized, which allows their bodies to stay in a relaxing posture. The average 'anus-tonose' lengths in gender-matched littermate $N e d d 4^{+/-}$and wild-type mice were similar on HFD (Figure 3.4A), suggesting that $N e d d 4^{+/-}$mice are leaner rather than smaller. Representative anatomical pictures of mice and dissected fat depots were shown in Figure 3.4B. Postmortem dissection for epididymal, inguinal subcutaneous white, and interscapular brown fat depots revealed significantly reduced accumulation of visceral and subcutaneous fat but not interscapular brown fat in HFD-fed $\mathrm{Nedd} 4^{+/-}$mice compared to controls (Figure 3.4C). Ratios of epididymal and inguinal subcutaneous WAT mass vs. body mass were $\sim 36 \%$ lower in $\mathrm{Nedd}^{+/-}(4.5 \pm 0.5 \%$ and $2.8 \pm 0.4 \%)$ relative to control mice $(7.0 \pm 0.1 \%$ and $4.4 \pm 0.5 \%)$ on HFD $(P<0.05)$ (Figure 3.4D and E). In addition, the leptin mRNA levels in epididymal WAT were $32.5 \%$ lower in Nedd ${ }^{+/-}$mice $(P<0.05)$ (Figure 3.4F), consistent with leptin as an indicator of body fat (Caro et al., 1996). We estimated the lean body mass index by the weight of non-fat tissues, such as the liver, heart, kidney, brain, and skeletal muscle (i.e., gastrocnemius and quadriceps),vs. body weight. Our data show that the ratio of lean body mass vs. body mass were similar between genotypes (Figure 3.4H).

On the ND, the ratios of lean body mass vs. body mass were similar between $N e d d 4^{+/-}$and wild-type mice (Figure 3.4G). The observed lack of apparent decrease in the weight gain in $N e d d 4^{+/-}$mice on ND (Figure 3.2A) was most likely because their increase in lean body mass compensated for the decrease in fat mass; fat mass only accounts for a small portion of body mass prior to middle age. These observations suggest that protection of $\mathrm{Nedd} 4^{+/-}$mice from diet-induced obesity, and even age-induced obesity potentially, could have resulted primarily from the reduced fat mass.

\subsubsection{Nedd4-haploinsufficient Mice Are Resistant to Several HFD-induced Deleterious Effects without Changes in Serum Lipid Profile}

The expansion of WAT in obesity can be a result of adipocyte hypertrophy (increase in cell size) and/or hyperplasia (increase in cell number) (de Ferranti and Mozaffarian, 2008; Sun et al., 2011). Histological analysis in the paraffin embedded epididymal WAT revealed a significant $25 \%$ smaller average cell size in $\mathrm{Nedd} 4^{+/-}$mice on HFD (Figure 3.5A and B). Cell size distribution analysis showed a greater frequency of small and midsized adipocytes and a lower frequency of large adipocytes in epididymal WAT from the HFD-fed $N e d d 4^{+/-}$mice, while the distribution curves in the 
A

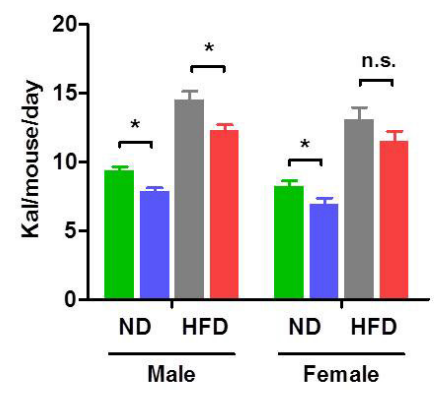

C

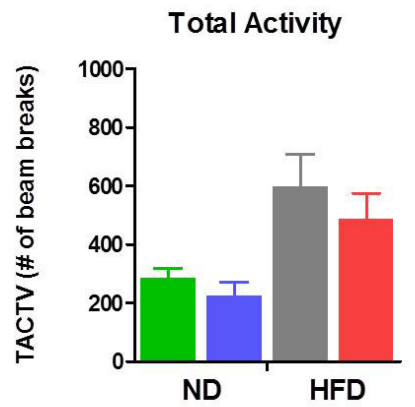

E

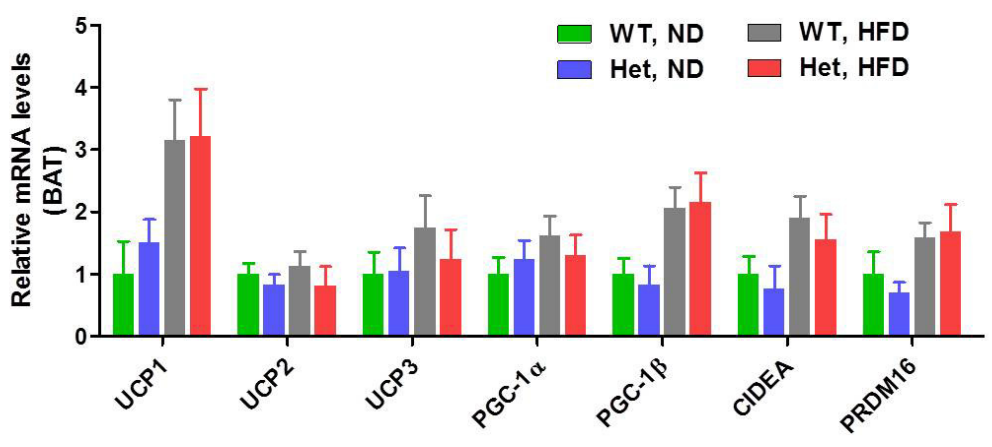

Figure 3.3 Nedd4-haploinsufficient Mice Exhibit Normal Physical Activity, Energy Intake, and Thermogenesis-related Gene Expression.

(A) Mice at ages 12-16 weeks were fed a known amount of food ad libitum. After $24 \mathrm{hr}$, the remaining food was weighed. Consumption of food (absolute food intake) was calculated by the difference. Food intake was monitored on a daily basis for a continuous 7-day period. Food intake was converted to energy intake by multiplying by the energy density in food. (B) Relative food intake was calculated as the absolute food intake per gram of body weight. (C and D) Animals were individually placed for $10 \mathrm{~min}$ in a novel open-field box equipped with beams that automatically measure total locomotor activity by beam breaks. (C) Total number of beam breaks indicates total activity. (D) Total distance traveled in inches. ND, $n=14-23$ per group; HFD, $n=8-10$ per group. (E) Relative mRNA levels of thermogenic genes in BAT in littermates WT and Het on ND or HFD were measured by qRT-PCR (ND, n=3 per group; HFD, n=5-6 per group). Data represent mean $\pm \mathrm{SEM} ; * P<0.05 ;$ n.s. no significance. 


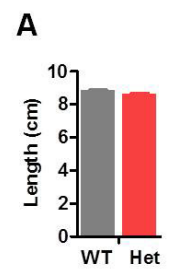

B

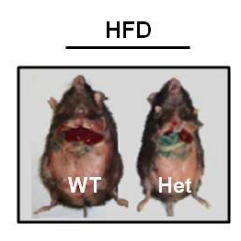

D

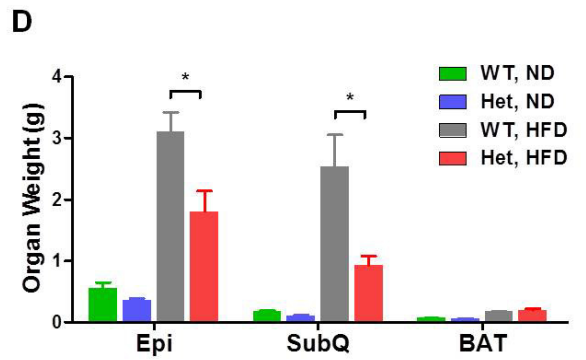

C

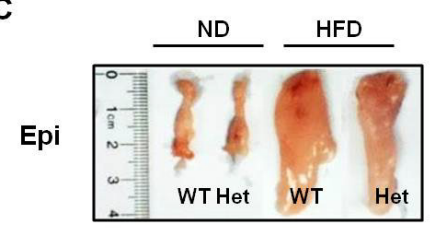

$\mathbf{E}$

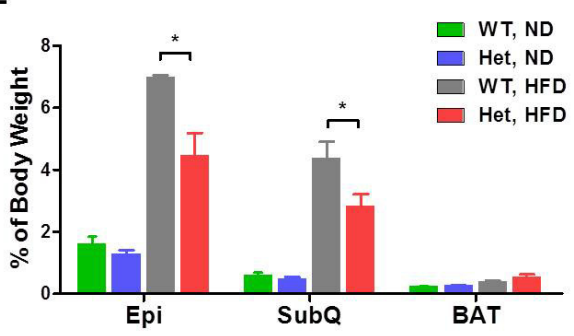

G

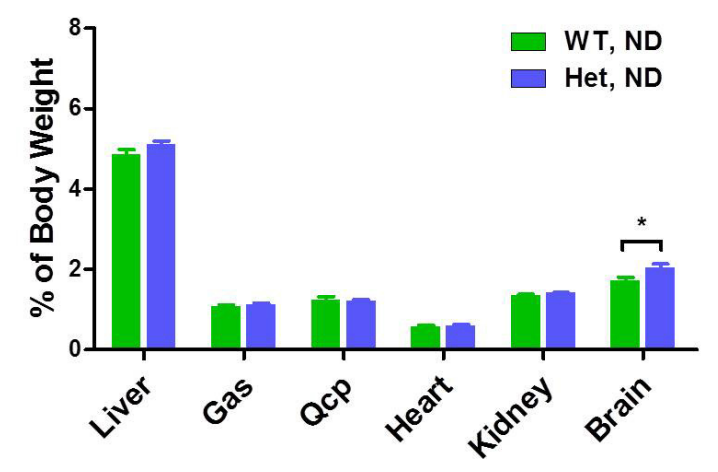

H
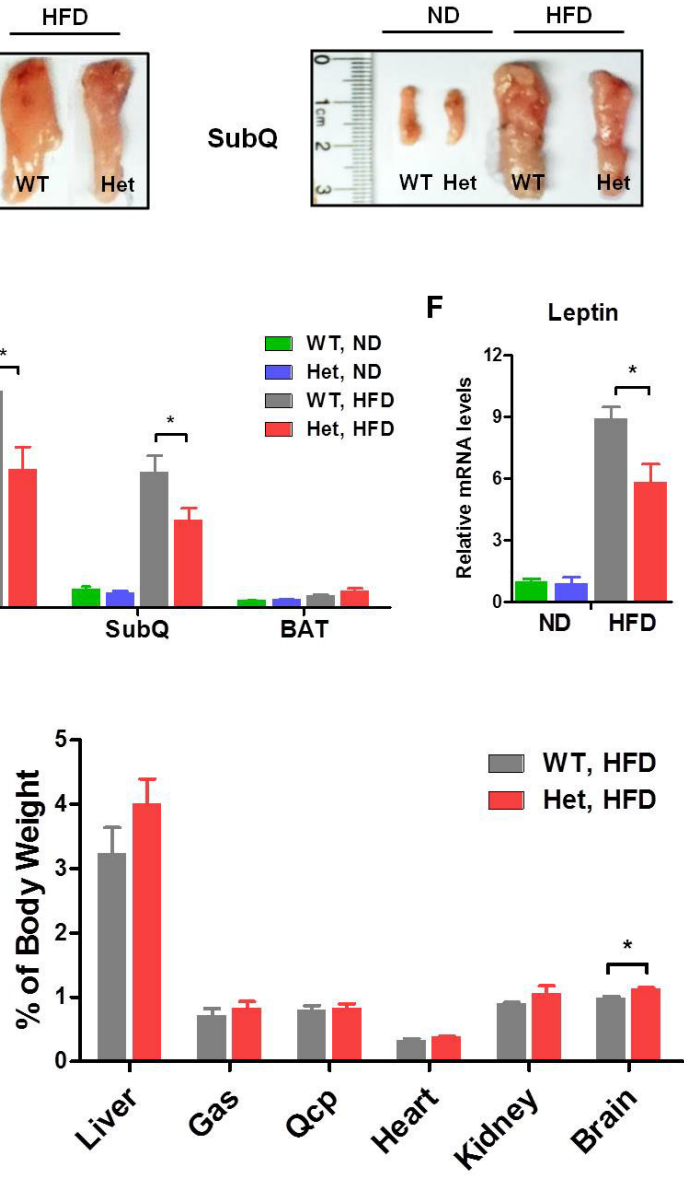

Figure 3.4 Nedd4-haploinsufficient Mice on HFD Have Reduced Fat Mass.

(A) Average 'anus-to-nose' lengths in gender-matched wild-type (WT) and $\mathrm{Nedd}^{+/-}(\mathrm{Het})$ littermates. $\mathrm{n}=5$ per group. (B) Representative anatomical views of mice fed a HFD. (C) Epididymal (Epi) and inguinal subcutaneous (SubQ) fat pads were dissected from WT and Het mice on ND or HFD. (D) Epi, SubQ and BAT depot weights in gender-matched littermates WT and Het at 22 weeks of age. (E) Percentage of fat pad weights to the body weights. $\mathrm{n}=5-7$ per group. (F) Leptin mRNA levels in Epi by qRT-PCR analysis (ND $n=3$ per genotype; HFD $n=5-6$ per genotype). ( $\mathrm{G}$ and $\mathrm{H})$ Percentage of organ weights to the body weights on ND $(\mathrm{G})$ or on HFD $(\mathrm{H})(\mathrm{n}=5-7$ per group). All data are mean $\pm \mathrm{SEM}$; $* P<0.05, * * P<0.01$ and $* * * P<0.001$. 
A

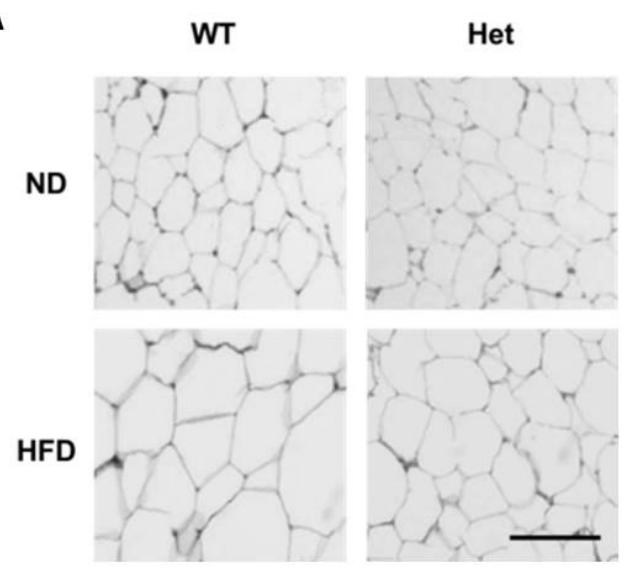

C

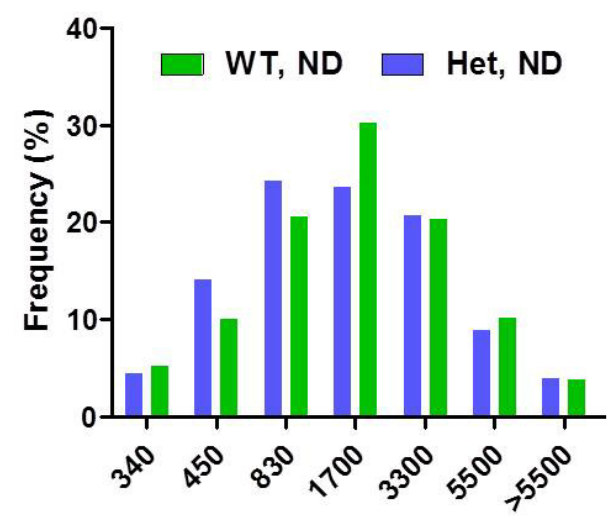

B

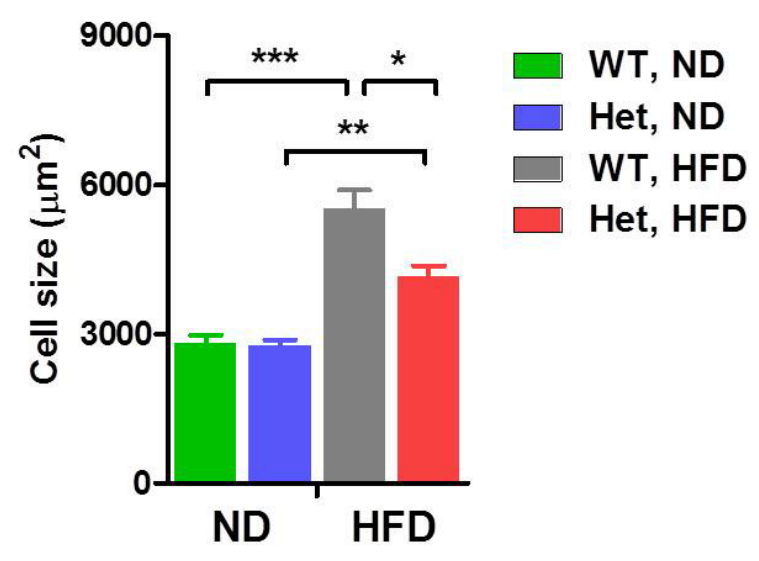

D

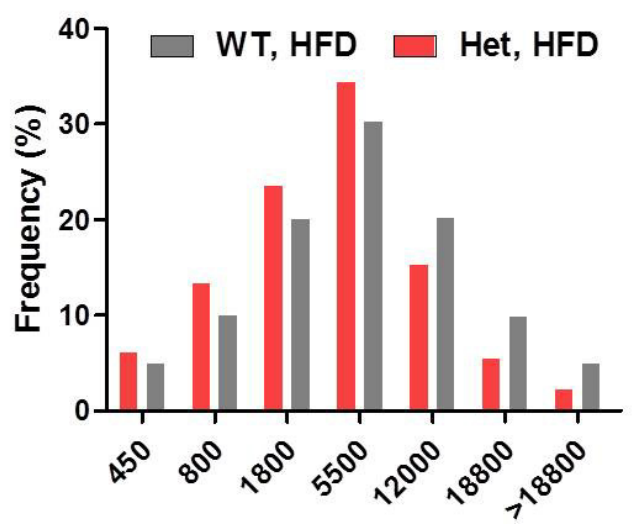

Figure 3.5 Nedd4-haploinsufficient Mice Are Protected against HFD-induced Adipocyte Hypertrophy.

(A) Representative H\&E staining of adipocytes. (B-D) Mean (B) and distribution (C and D) of adipocyte size in epididymal adipose tissue of wild-type (WT) and $\mathrm{Nedd}^{+/-}$(Het) littermates on ND (C) or HFD (D) ( $n=3-4$ per group). The area of adipocytes was measured for 300-400 cells per mouse using ImageJ software with "Adipocytes Tools" plug-in. Scale bar $=100 \mu \mathrm{m}$. All data are mean $\pm \mathrm{SEM} ;{ }^{*} P<0.05,{ }^{*} P<0.01, * * * P<0.001$. 
ND-fed $\mathrm{Nedd} 4^{+/-}$and wild-type mice were almost overlapping (Figure 3.5C and D). Hence, NEDD4 downregulation in obese mice was associated with reduced adipocyte volume indicating lower degrees of lipid accumulation within adipocytes.

Chronic HFD leads to TG accumulation in liver called non-alcoholic fatty liver or hepatic steatosis (Motomura et al., 2006). After 16 weeks of HFD, hepatocytes of wildtype mice were filled with multiple large lipid droplets, whereas $\mathrm{Nedd4^{+/- }}$ littermates displayed significantly smaller and fewer lipid droplets as detected by hematoxylin and eosin stain or oil red O stain (Figure 3.6A and B). These data indicate protection from hepatic steatosis in $\mathrm{Nedd} 4^{+/-}$mice.

Low-grade inflammation invariably accompanies significant obesity (Wellen and Hotamisligil, 2003). As expected, we saw dramatic HFD induced increases in mRNA levels of several pro-inflammatory markers (MIP-1 $\alpha$, MCP-1, TNF $\alpha$, F4/80, and IL-1 $\beta$ ) within the WAT of wild-type mice. TNF- $\alpha$ and F4/80 are M1 macrophage markers, and MIP-1 $\alpha$ and MCP-1 represent two major chemokines essential for recruiting M1 macrophages (Mantovani et al., 2004). The observed lower transcriptional expression of these markers in HFD-fed $N e d d 4^{+/-}$mice compared to wild-type littermates may indicate reduced M1 macrophage infiltration in the HFD-fed $\mathrm{Ne} d d 4^{+/-}$mice. IL4 and antiinflammatory IL10, two TH2 cytokines, can induce M2 macrophage polarization (Mantovani et al., 2004). Interestingly, IL4 and IL10 mRNA levels were not significantly different in WAT between genotypes, suggesting that the attenuation of lipid-induced inflammation may be independent of M2 action (Figure 3.7). This is consistent with the cell size distribution data that small adipocyte $\left(<450 \mu \mathrm{m}^{2}\right)$ percentages in HFD-fed $\mathrm{Nedd} 4^{+/-}$and wild-type mice were similar (Figure 3.5D), since M2 macrophages mainly present in lean adipose tissue (Fujisaka et al., 2009). Of note, NEDD4 protein levels per se were unaltered by HFD in WAT, skeletal muscle, or hypothalamus (Figure 3.8A-C).

Dietary obesity is usually accompanied with enhanced basal lipolysis, resulting in increased serum cholesterol, TG, glycerol, and FFA levels. However, we found unaltered serum lipid profile in ND- or HFD-fed $\mathrm{Nedd}^{+/-}$mice relative to their respective controls (Table 3.1).

\subsubsection{Nedd4-haploinsufficient Mice Retain Insulin Sensitivity in WAT despite HFD}

To examine whole body insulin sensitivity, we performed GTT and ITT on ageand gender-matched mice at approximately age 22 weeks. In ND-fed $\mathrm{Nedd4^{+/- }}$ vs. controls, we observed decreased insulin sensitivity during ITT (Figure 3.9B) and a small compensatory increase in the serum insulin levels under both fed $(3.65 \pm 0.28 \mathrm{ng} / \mathrm{mL}$ vs. $2.77 \pm 0.27 \mathrm{ng} / \mathrm{mL}, P<0.01)$ and fasted $(1.11 \pm 0.10 \mathrm{ng} / \mathrm{mL}$ vs. $0.73 \pm 0.08 \mathrm{ng} / \mathrm{mL}$, $P<0.01)$ states (Figure 3.9D and E). Consistently, serum insulin levels in ND-fed $\mathrm{Nedd} 4^{+/-}$during GTT is slightly higher than that in the wild-type mice $(P<0.05)$ (Figure 3.9C). Despite these defects, ND-fed $\mathrm{Nedd} 4^{+/-}$mice were paradoxically glucose tolerant with normal basal blood glucose level (Figure 3.9A). Collectively, the data suggest that 
A
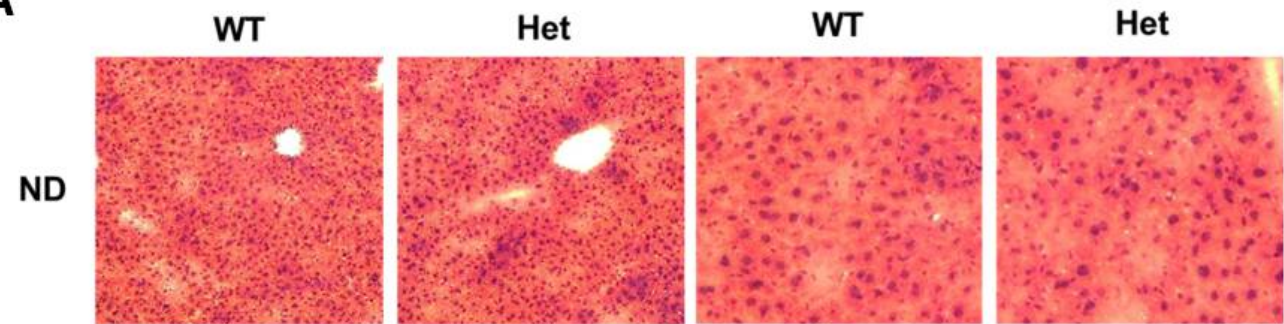

HFD
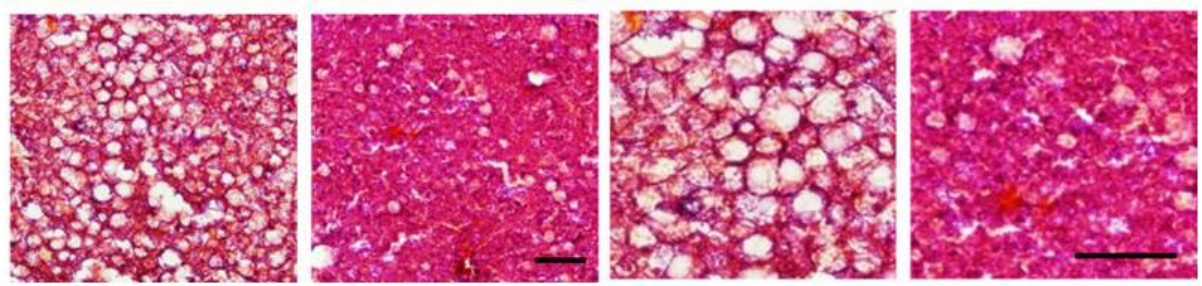

B
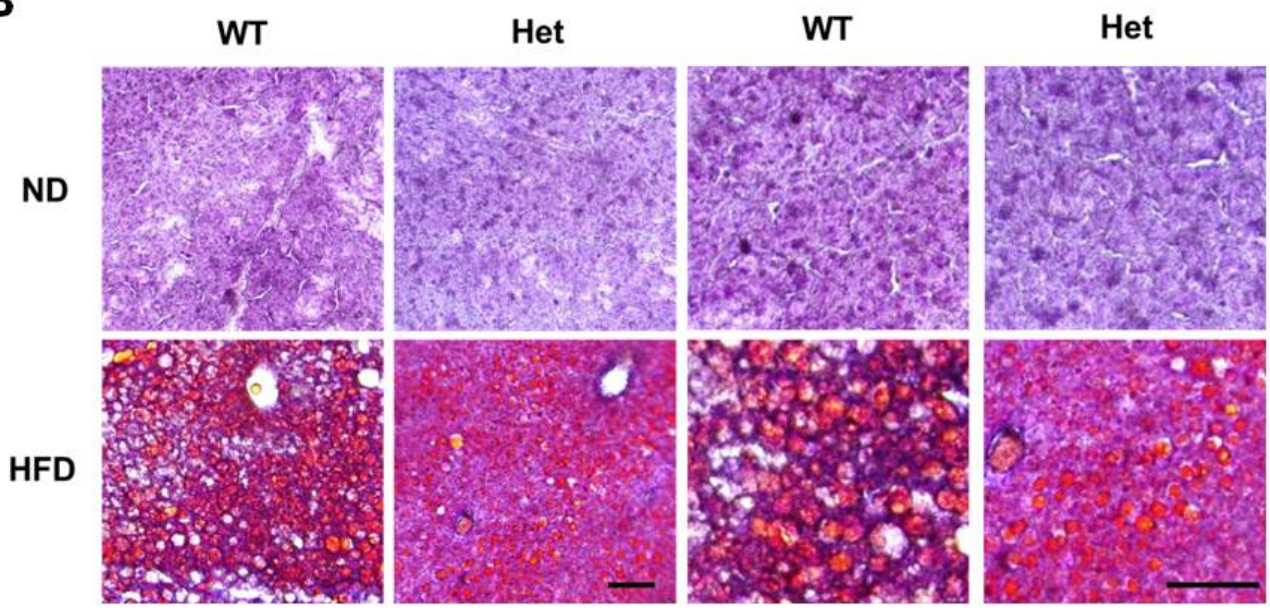

Figure 3.6 Nedd4-haploinsufficient Mice Are Protected against HFD-induced Hepatic Steatosis.

(A) Representative H\&E staining of liver in littermates wild-type (WT) and Nedd4 $4^{+-}$ (Het) on ND or HFD ( $n=3)$. Sections were stained with hematoxylin for nuclei and counterstained with eosin. (B) Representative oil red $\mathrm{O}$ staining in liver sections from WT and Het mice on ND or HFD ( $n=3)$. Fixed cryosections were stained with freshly prepared oil red $\mathrm{O}$ working solution for $15 \mathrm{~min}$ and counterstained for nuclei with hematoxylin. Scale bar $=100 \mu \mathrm{m}$. 

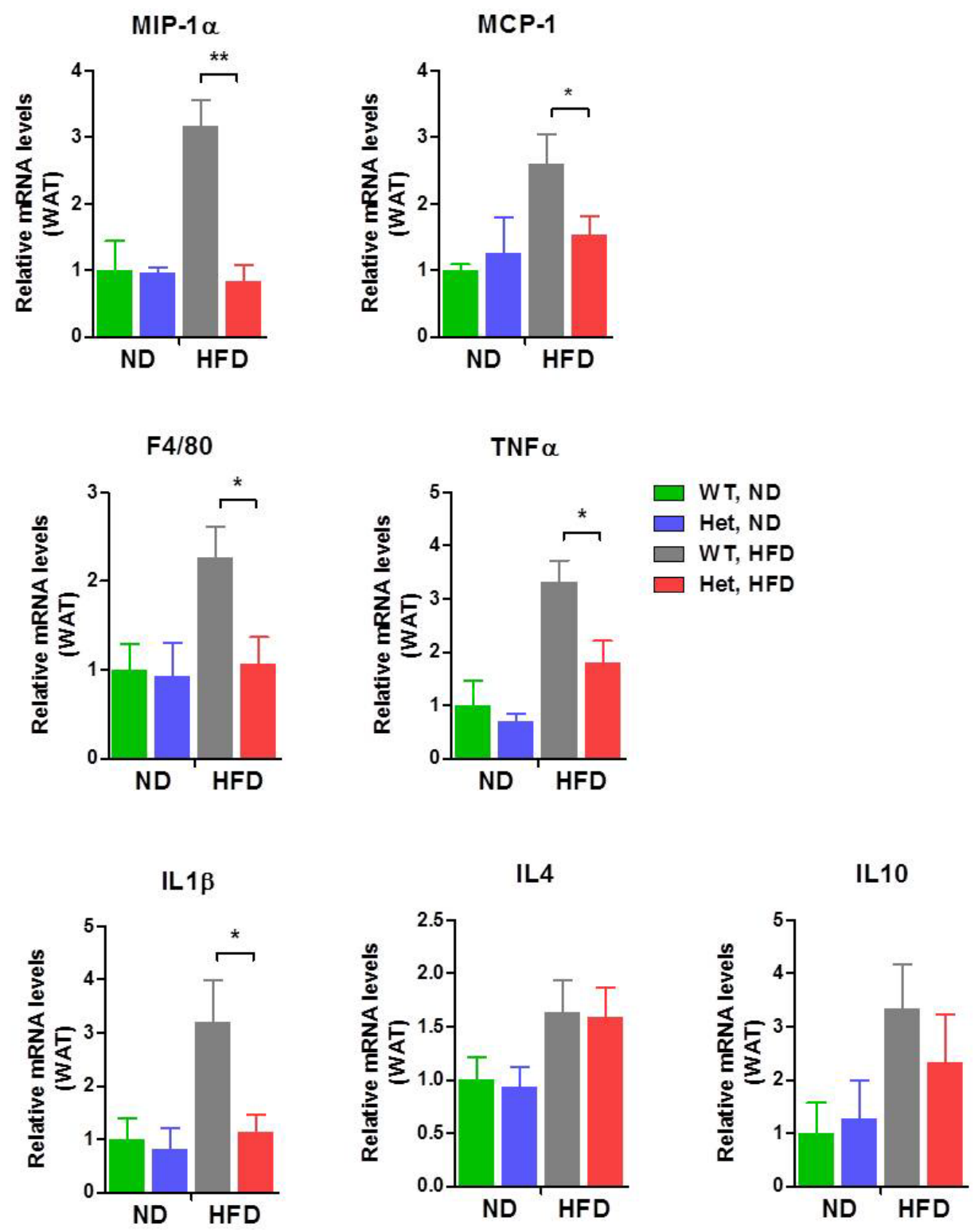

Figure 3.7 Nedd4-haploinsufficient Mice Are Protected against Lipid-induced Inflammation.

Relative transcriptional levels of inflammatory markers in epididymal fat in littermates wild-type (WT) and $\mathrm{Nedd}^{+/-}$(Het) on ND or HFD were measured by qRT-PCR. ND n=3 per genotype; HFD ( $\mathrm{n}=5-6$ per genotype). Around $50 \mathrm{mg}$ of adipose tissue was lysed in 1 $\mathrm{ml}$ of Trizol for RNA isolation. Around $1 \mu \mathrm{g}$ of total RNA was reverse transcribed into cDNAs which were subsequently used for qRT-PCR detection with intron-spanning primers. All data are mean $\pm \mathrm{SEM} ; * P<0.05, * * P<0.01$. 
A
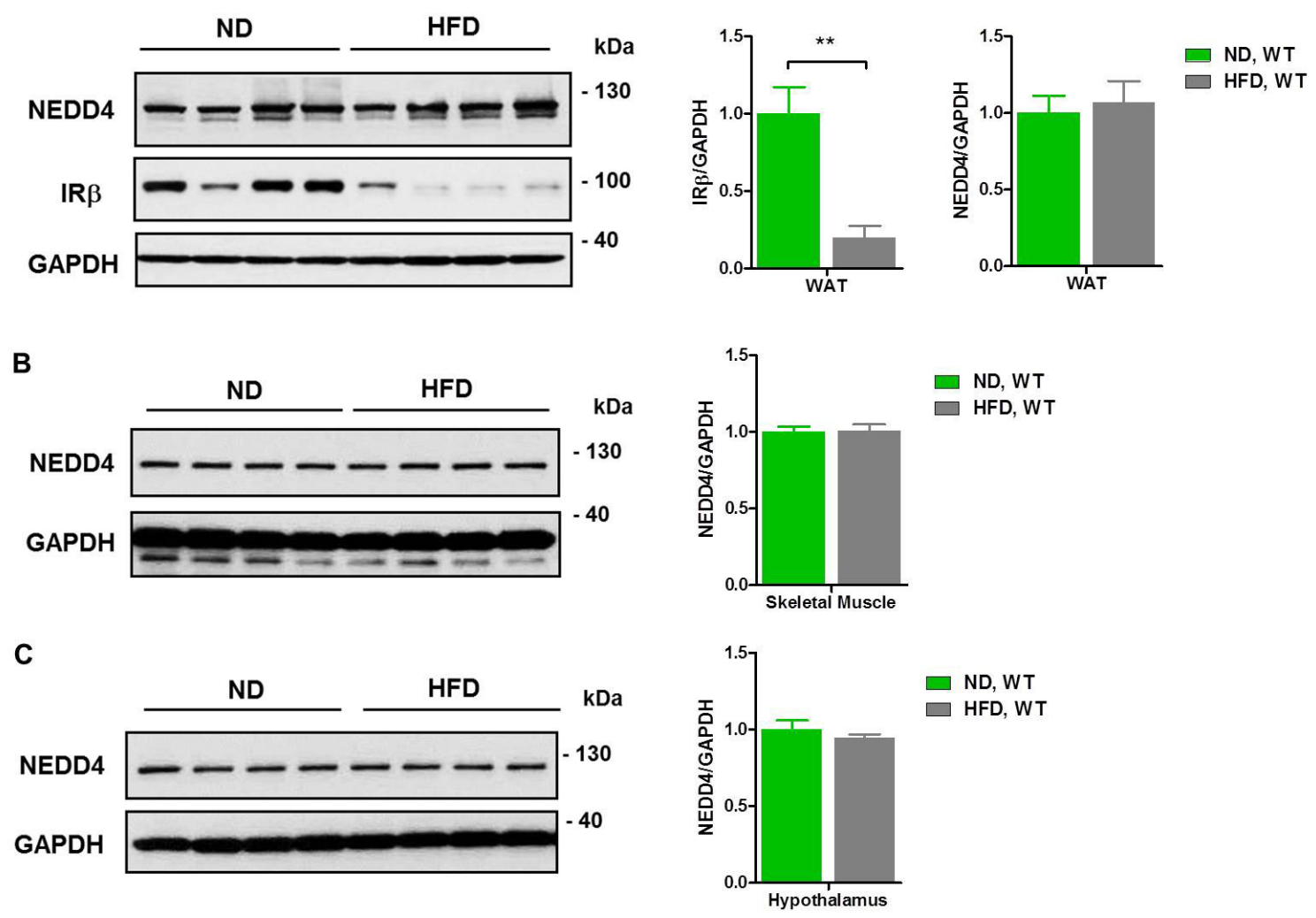

Figure 3.8 NEDD4 Protein Levels Are Not Altered by HFD Feeding.

Immunoblot analysis of NEDD4 protein levels in epididymal WAT (A), gastrocnemius muscle (B) and hypothalamus (C) tissue lysates from WT mice fed on ND or HFD at age 36 weeks. The IR $\beta$ levels in WAT served as a positive control of the effect of HFD. The GAPDH levels served as loading controls. 
Table 3.1 Metabolic Parameters in Serum or Blood.

\begin{tabular}{|c|c|c|c|c|c|c|c|c|c|c|}
\hline \multirow[b]{2}{*}{ Measurements } & \multicolumn{2}{|l|}{ WT, ND } & \multicolumn{2}{|l|}{ Het, ND } & \multirow[b]{2}{*}{ Statistics } & \multicolumn{2}{|l|}{$\begin{array}{l}\text { WT, HFD } \\
\end{array}$} & \multicolumn{2}{|l|}{ Het, HFD } & \multirow[b]{2}{*}{ Statistic } \\
\hline & Mean \pm SEM & $\mathbf{n}$ & Mean \pm SEM & $\mathbf{n}$ & & Mean \pm SEM & $\mathbf{n}$ & Mean \pm SEM & $\mathbf{n}$ & \\
\hline \multicolumn{11}{|l|}{ Glucose (mM) } \\
\hline Fed & $11.46 \pm 0.49$ & 12 & $11.84 \pm 0.36$ & 12 & n.s. & $12.95 \pm 0.43$ & 12 & $13.24 \pm 0.56$ & 12 & n.s. \\
\hline Fasted & $8.02 \pm 0.57$ & 12 & $8.73 \pm 0.37$ & 12 & n.s. & $10.29 \pm 0.38$ & 12 & $9.52 \pm 0.79$ & 12 & n.s. \\
\hline Cholesterol (mM) & & & & & & & & & & \\
\hline Fed & $4.30 \pm 0.37$ & 10 & $4.40 \pm 0.31$ & 10 & n.s. & $6.37 \pm 0.45$ & 10 & $6.73 \pm 0.45$ & 10 & n.s. \\
\hline $\begin{array}{l}\text { Fasted } \\
\text { TG (mM) }\end{array}$ & $2.87 \pm 0.50$ & 10 & $2.65 \pm 0.62$ & 10 & n.s. & $3.48 \pm 1.07$ & 10 & $2.20 \pm 1.41$ & 10 & n.s. \\
\hline Fed & $2.26 \pm 0.31$ & 10 & $2.65 \pm 0.21$ & 10 & n.s. & $2.74 \pm 0.20$ & 10 & $2.79 \pm 0.35$ & 10 & n.s. \\
\hline $\begin{array}{l}\text { Fasted } \\
\text { Glycerol (mM) }\end{array}$ & $1.83 \pm 0.18$ & 10 & $1.81 \pm 0.20$ & 10 & n.s. & $2.33 \pm 0.39$ & 10 & $2.53 \pm 0.68$ & 10 & n.s. \\
\hline Fed & $3.87 \pm 0.32$ & 8 & $3.86 \pm 0.29$ & 7 & n.s. & $6.01 \pm 0.35$ & 9 & $5.85 \pm 0.52$ & 6 & n.s. \\
\hline $\begin{array}{l}\text { Fasted } \\
\text { FFA (mM) }\end{array}$ & $4.92 \pm 0.44$ & 8 & $5.25 \pm 0.31$ & 7 & n.s. & $5.05 \pm 0.63$ & 9 & $3.76 \pm 0.59$ & 6 & n.s. \\
\hline Fed & $0.20 \pm 0.02$ & 8 & $0.20 \pm 0.02$ & 7 & n.s. & $0.28 \pm 0.02$ & 9 & $0.28 \pm 0.02$ & 6 & n.s. \\
\hline Fasted & $0.17 \pm 0.04$ & 8 & $0.17 \pm 0.03$ & 7 & n.s. & $0.18 \pm 0.07$ & 9 & $0.18 \pm 0.08$ & 6 & n.s. \\
\hline
\end{tabular}

Notes: Blood glucose and lipid levels in serum from fed and fasted mice were measured by a glucose meter or respective assay kits. Fed glucose levels are higher in HFD groups compared to respective group on ND (WT, $P<0.05 ; N e d d 4^{+/-}, P<0.05$ ). Fasting glucose and TG levels are higher in WT, HFD compared to WT, ND $(P<0.01)$. Fed cholesterol, TG, glycerol, and FFA levels are higher in HFD groups compared to respective group on ND (WT, $P<0.001 ; N e d d 4^{+/}, P<0.001$ ). Each group contains relatively equal numbers of male and female. Mice were tested around 22 weeks of age. WT, wild-type mice; Het, Nedd ${ }^{+/-}$mice. All data are means \pm SEM; n.s. no significance. 
A

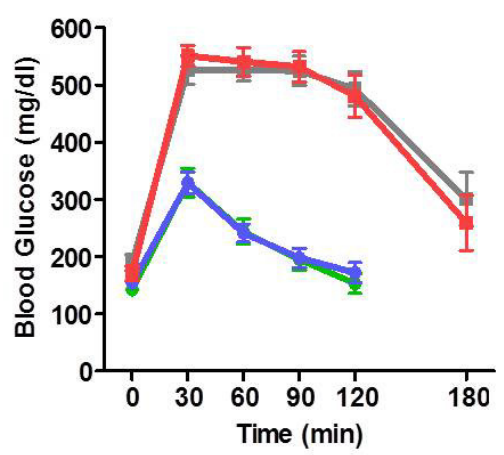

C

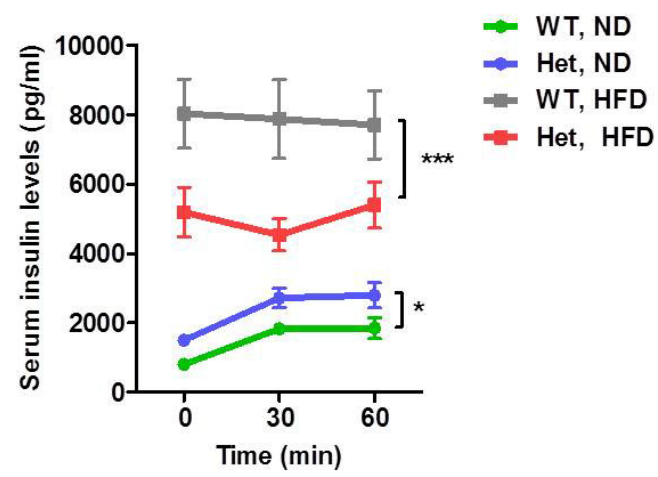

D

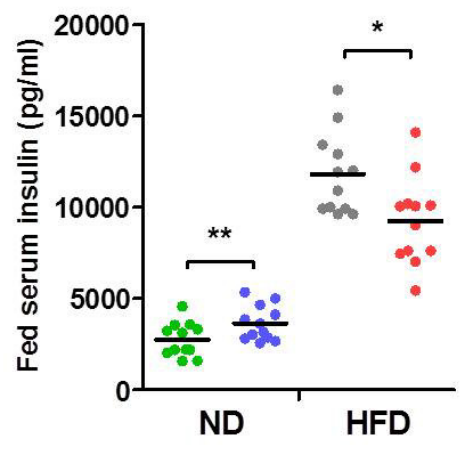

E
B

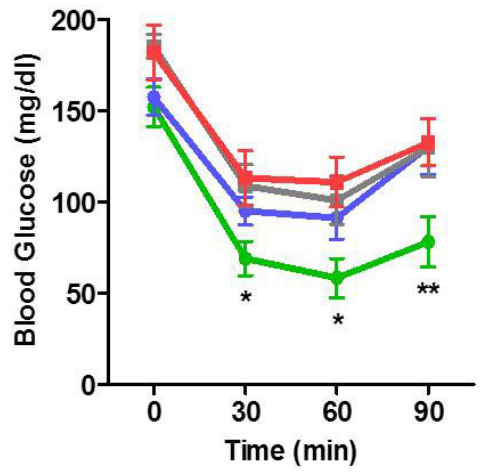

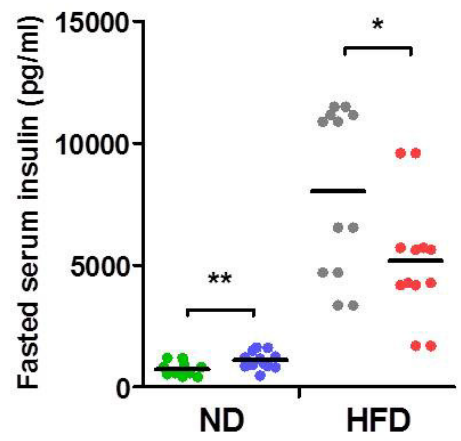

Figure 3.9 GTT, ITT, and Serum Insulin Levels.

(A) No significant difference in glucose clearance ability between two genotypes $(n=12-$ 19 per group). (B) Under normal diet (ND), Nedd $4^{+/-}$(Het) mice were less responsive to insulin compared to wild-type (WT) littermate controls based on glucose clearance rate ( $n=12-19$ per group). (C) Serum insulin levels during GTT ( $n=12-19$ per group with both genders). For GTT, mice were fasted overnight and i.p. injected with $1.5 \mathrm{~g} / \mathrm{kg}$ of glucose. For ITT, mice were fasted for $4 \mathrm{hr}$ and i.p. injected with $0.75 \mathrm{U} / \mathrm{kg}$ of insulin. Fed (D) and fasted (E) serum insulin levels in WT and Het mice on ND or HFD. All data are mean $\pm \mathrm{SEM} ; * P<0.05, * * P<0.01, * * * P<0.01$. 
ND-fed $\mathrm{Ne}_{\mathrm{d}} 4^{+/-}$mice possess only mildly decreased insulin sensitivity, which can be compensated by increased insulin secretion.

In wild-type mice, HFD induced profound glucose intolerance, insulin resistance associated with a five-fold increase in the basal circulating insulin concentration, and failure of glucose-stimulated insulin secretion (Figure 3.9A-C). Most surprising, chronically HFD-fed $\mathrm{Nedd} 4^{+/}$mice displayed same degree of glucose intolerance (Figure 3.9A and B) despite lower circulating insulin levels (Figure 3.9C-E), compared to HFDfed controls (fed, $9.23 \pm 0.69 \mathrm{ng} / \mathrm{mL}$ vs. $11.8 \pm 0.65 \mathrm{ng} / \mathrm{mL}, P<0.05$; fasted, $5.19 \pm 0.72$ $\mathrm{ng} / \mathrm{mL}$ vs. $8.03 \pm 0.99 \mathrm{ng} / \mathrm{mL}, P<0.05)$, suggesting that the HFD-fed Nedd ${ }^{+/-}$mice required less circulating insulin to maintain euglycemia.

To delineate insulin sensitivity in specifically responsive tissues, we analyzed insulin-stimulated signaling in WAT, skeletal muscle, and liver. Western blot analysis of in vivo insulin signaling in fat, skeletal muscle, and liver demonstrated reduced phosphorylation of IR and protein kinase B/AKT (PKB/AKT) evoked by a single i.p. insulin injection in ND-fed Nedd $4^{+/-}$mice (Figure 3.10A-C). Nedd $4^{+/-}$mice on HFD displayed enhanced tyrosine phosphorylation of IR and their downstream PK B/AKT only in WAT (Figure 3.10D), but not in skeletal muscle or liver (Figure 3.10E and F).

\subsection{Summary}

NEDD4 is a HECT-type E3 ligase that has been implicated in the downregulation of several activated receptor tyrosine kinases in vitro. Genetic knockout of NEDD4 led to reduced IGF-1 signaling, suggesting that the role of NEDD4 in this system is more complex. Because NEDD4 knockout mice have major embryonic defects, most of the current studies regarding NEDD4 function are restricted to mouse embryonic development. We found that $\mathrm{Ned} d 4^{+/-}$mice were devoid of gross morbidity or mortality and therefore can be used for investigating NEDD4 function in adult and/or aging stage. Our preliminary studies confirmed several reported substrates of NEDD4 in neuronal culture system, such as PTEN and IGF-1R, both of which have central functions in insulin/IGF-1 signaling. To study the NEDD4's role in metabolic regulation, we challenged $\mathrm{Nedd}^{+/-}$mice with a HFD (60\% of calories from fat) for 30 weeks. By monitoring their body weights weekly, we found a significant difference in weight gain between $\mathrm{Ned} d 4^{+/-}$and wild-type mice.

Further characterization revealed that the loss of HFD-provoked weight gain in $N e d d 4^{+/-}$mice was due to reduced fat content, especially the mass of visceral and subcutaneous fat. Although the absolute weights of other non-fat organs were lower in $N e d d 4^{+/-}$mice than in controls, their weights were in proportion to their total body weights. Histological analysis of adipose tissue showed smaller adipocyte size in $\mathrm{Nedd4^{+/ }}$ mice. The associated complications, such as lipid-induced hepatic steatosis and inflammation, were also attenuated in HFD-fed $\mathrm{Nedd} 4^{+/-}$mice. These data suggest that $N e d d 4^{+/-}$mice have better ability to attain fat balance upon energy surplus, and therefore, resist to obesity development. 
Figure 3.10 In vivo Insulin Signaling in WAT, Skeletal Muscle, and Liver. Western blot analysis of phosphorylated and total IR $\beta$ and AKT protein levels in WAT (epididymal) (A and D), skeletal muscle (gastrocnemius) (B and E), and Liver (C and F) in wild-type (WT) and Nedd4 ${ }^{+/}$(Het) mice on ND (A-C) or HFD (D-F) with protein quantification ( $\mathrm{n}=5-7$ per group). Mice at age around 22 weeks were fasted overnight and i.p. injected with $5 \mathrm{U} / \mathrm{kg}$ of insulin and waited for $15 \mathrm{~min}$ before being euthanized. Tissues were quickly dissected and snap-frozen until use. All data are mean $\pm \mathrm{SEM}$; $* P<0.05, * * P<0.01$. 
A

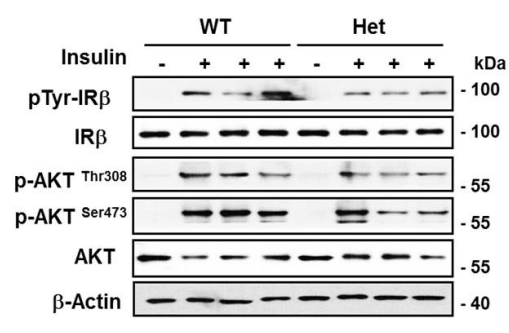

D
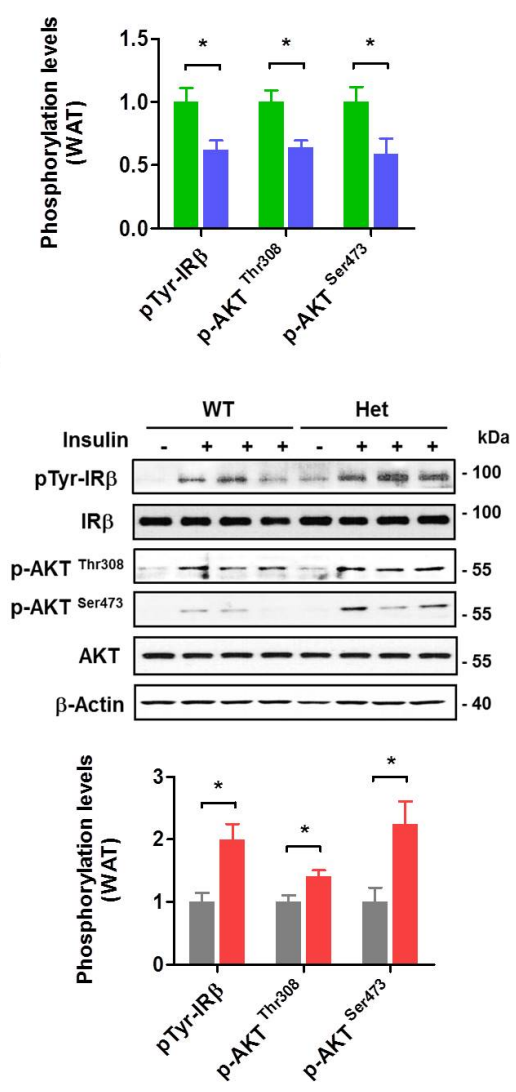

B

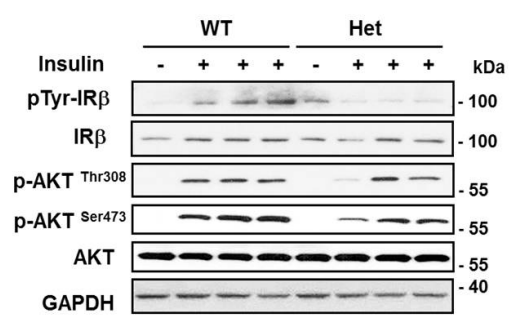

E
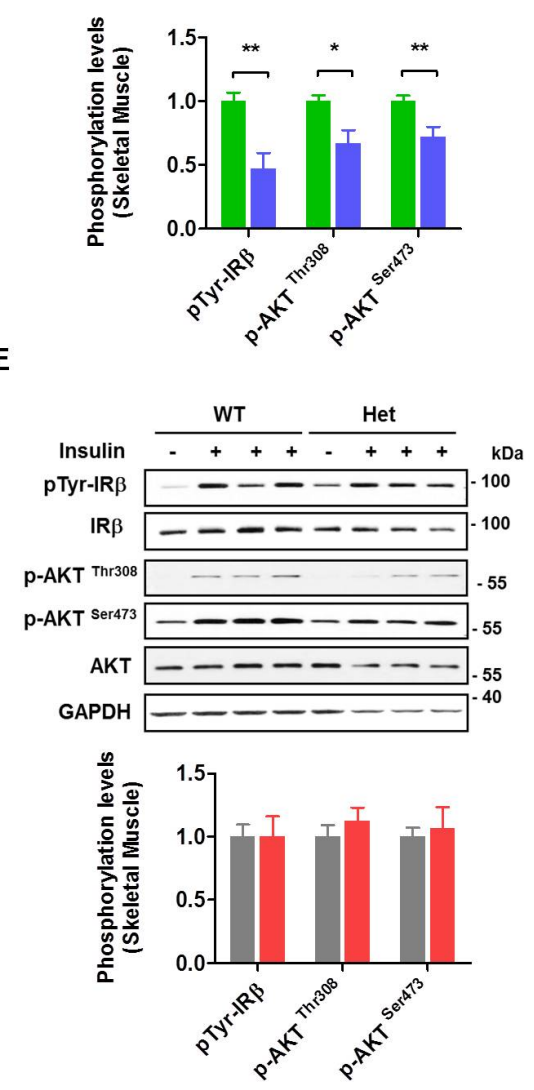

C
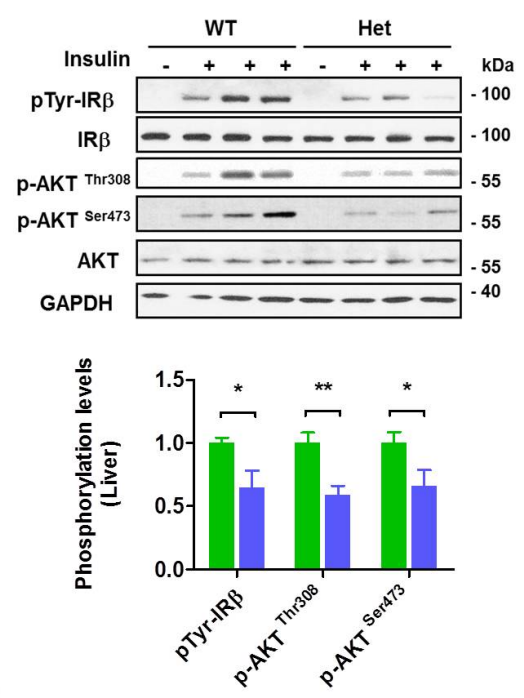

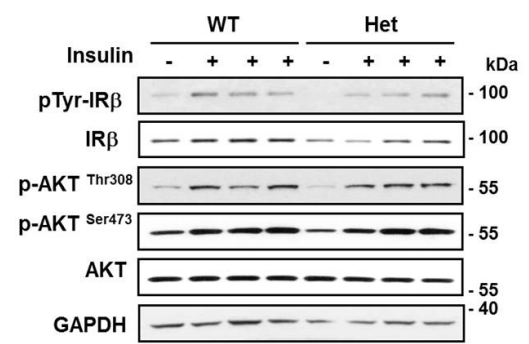

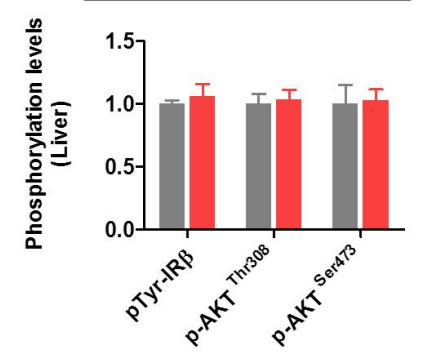


In response to HFD, partial loss of NEDD4 function was associated with reduced circulating insulin levels and selective insulin sensitivity improvement in WAT, but not in skeletal muscle or liver, despite unalleviated systemic impairment in glucose homeostasis. These data suggest that NEDD4 may partially mediate obesity-associated insulin resistance. Paradoxically, NEDD4 was required for maintaining normal insulin signaling under basal conditions (fed on ND), as $\mathrm{Nedd}^{+/-}$mice on ND displayed reduced insulin signaling in several insulin-sensitive organs (WAT, skeletal muscle, and liver). Taken together, these data imply that NEDD4 may have distinct physiological and pathophysiological roles in insulin system.

In addition to glucose tolerance, $N e d d 4^{+/-}$mice exhibited similar serum lipid concentrations to those of control mice with or without HFD challenge, suggesting that NEDD4 has minor roles in regulating glucose homeostasis and blood lipid turnover.Also, HFD did not affect NEDD4 expressional levels in several metabolic tissues (hypothalamus, skeletal muscle, WAT).

In summary, Nedd4 haploinsufficiency protects against HFD-induced obesity, hyperinsulinemia, adipocyte hypertrophy, hepatic steatosis, and pro-inflammatory response. However, partial loss of NEDD4 function does not alleviate obesity-associated insulin resistance, glucose intolerance, and hyperlipidemia, decoupling obesity from several of its associated pathologies. Although the reason why $N e d d 4^{+/-}$mice manifest these decoupling effects is unclear, our studies may provide experimental evidence for clinical heterogeneity seen in metabolic syndrome. 


\section{CHAPTER 4. MECHANISTIC STUDIES}

\subsection{Introduction}

E3 ligases that possess the ability of selecting specific proteins for ubiquitin conjugation are important regulators in protein degradation process. NEDD4 is the prototypic member in NEDD4 protein family of E3 ligases which contains a C2 domain, 3-4 WW domains, and a catalytic HECT domain. During the process of ubiquitination, the ubiquitin-conjugated E2 binds to the C-terminal HECT domain, initiating a thiol-ester exchange to transfer the ubiquitin from $\mathrm{E} 2$ to a catalytic cysteine residue within the $\mathrm{C}$ terminal lobe of the HECT domain (Ingham et al., 2004). HECT domain, therefore, transiently receive ubiquitin before its transfer to the substrate. Repetitive occurrence of this thiol-ester exchange produces a polyubiquitin chain which targets the substrate protein for lysosomal or proteasomal degradation (Wang et al., 1999a). While the C2 domain regulates cellular translocation of NEDD4, the multiple WW domains which bind predominantly to proline-rich motifs (PPxY, PPLP, PR, and phosphoserine/threonine residues that precede a proline residue) are mainly responsible for substrate selection by NEDD4 (Ingham et al., 2004).

Two mammalian non-visual arrestins ( $\beta$-arrestin 1 and 2$)$ and the arrestin domaincontaining proteins (ARRDC2-4) have been reported to function primarily as adaptor proteins between E3 ligase NEDD4 and $\beta_{2}$-AR (Han et al., 2013b). No proline-rich motifs were found in $\beta$-arrestins; mutation in WW domains of NEDD4 did not affect the recruitment of NEDD4 to $\beta_{2}$-AR, suggesting that non-canonical binding exists between NEDD4 and $\beta$-arrestins (Shenoy et al., 2008). Unlike $\beta$-arrestins, a PPxY motif was found in ARRDCs. The ARRDC3 protein interacts with NEDD4 through two conserved PPxY motifs (Nabhan et al., 2010). Interestingly, ARRDC3-deficient mice also displayed

partial resistance to obesity presumably via modulating $\beta$-adrenergic signaling (Patwari et al., 2011)

In Chapter 3 we have carefully characterized the metabolic phenotypes in $N e d d 4^{+/-}$mice. We found that $N e d d 4^{+/-}$mice after HFD feeding elicited reduced adiposity, due mainly to the smaller visceral and subcutaneous fat depots. In the current chapter, we seek to investigate the underlying mechanisms. We examined whether the lipolytic efficiency was altered in HFD-fed $\mathrm{Ned} d 4^{+/-}$mice as they manifested reduced lipid storage and the $\beta_{2}$-AR is an essential mediator of lipolysis process in fat. Here, we also investigated potential regulation of PPAR $\gamma$ by NEDD4. Because it has been widely accepted that PPAR $\gamma$ plays fundamental regulatory roles in adipose tissue biology and pathology: PPAR $\gamma$ mediates not only adipogenesis but also HFD-induced adipocyte hypertrophy (Kubota et al., 1999), two important processes controlling adipose tissue volume. 


\subsection{Results}

\subsubsection{Knockdown of NEDD4 Accelerates Lipolysis}

Lipolysis rate in response to i.p. injection of isoproterenol (a non-selective $\beta$-AR agonists) in vivo was similar between ND-fed $\mathrm{Nedd}^{+/-}$and wild-type mice. However, the lipolysis rate in the HFD-fed $\mathrm{Ned} d 4^{+/-}$mice was significantly higher than controls (Figure 4.1A). To confirm this in vivo finding, we assayed lipolysis ex vivo in purified primary adipocytes. Whereas HFD greatly inhibited the isoproterenol-stimulated glycerol release in wild-type adipocytes, $N e d d 4^{+/}$adipocytes remained highly sensitive to $\beta$-AR stimulation. Furthermore, preincubation with insulin (a potent anti-lipolytic hormone) of primary adipocytes isolated from HFD-fed $\mathrm{Ned} d 4^{+/-}$mice completely blocked isoproterenol-stimulated glycerol release. This effect was not observed in controls and was consistent with respective levels of insulin signaling in WAT. To assess whether $\beta_{1}$ $\mathrm{AR}$ and/or $\beta_{2}$-AR mediated this effect, we preincubated the adipocytes with ICI-118,551 (a selective $\beta_{2}$-AR antagonist) or CGP-20712 (a selective $\beta_{1}$-AR antagonist). ICI-118,551 completely blocked the isoproterenol-stimulated lipolytic response observed in primary adipocytes derived from HFD-fed $\mathrm{Ned} d 4^{+/-}$mice. CGP-20712 attained only partial inhibition of lipolysis, suggesting that $\beta_{2}$-AR primarily mediates the enhancement of isoproterenol-induced lipolysis related to NEDD4 loss of function. In addition, treating primary adipocytes with other selective $\beta_{1}$-AR or $\beta_{2}$-AR agonists (xamoterol and salmeterol, respectively) confirmed $\beta_{2}$-AR as the predominant mediator (Figure 4.1B).

As mice age, their growth rates decrease and fatty acids are largely stored in the adipose tissue in the form of TGs, mimicking hyperlipidemia in clinical obesity. To investigate the effect of age on lipolysis, a set of age- and gender-matched $\mathrm{Nedd}^{+/-}$and wild-type mice were placed on ND, then followed to age roughly 18 months. We obtained epididymal fat tissue explants or purified primary adipocytes to assess lipolysis ex vivo. Isoproterenol stimulated significantly higher lipolysis in fat explant from aged $\mathrm{Nedd} 4^{+/-}$mice, as early as $30 \mathrm{~min}$ after incubation (Figure 4.1D). Isoproterenol and salmeterol, but not xemoterol, stimulated higher lipolysis rates in adipocytes from aged $\mathrm{Nedd} 4^{+/-}$mice, confirming accelerated lipolysis in aged $\mathrm{Nedd} 4^{+/-}$mice and a primary role for $\beta_{2}-A R$ in this regulation (Figure 4.1C).

\subsubsection{Knockdown of NEDD4 Increases $\beta_{2}$-AR Abundance at the Plasma Membrane}

Prolonged stimulation with agonists has been shown to downregulate $\beta_{2}-A R$, via endocytosis and the subsequent lysosomal degradation of active receptors. Recent work based on in vitro overexpression suggests NEDD4 is an E3 ligase involved in $\beta_{2}$-AR protein degradation (Nabhan et al., 2010; Shenoy et al., 2008). To validate the physiological impact of NEDD4 on $\beta_{2}$-AR regulation in vivo - in the context of lipolysis, - we first examined whether NEDD4 knockdown increases $\beta_{2}$-AR abundance. Whole cell lysates and crude membrane fractions prepared from WAT from HFD-fed $\mathrm{Nedd} 4^{+/-}$and wild-type mice were analyzed by immunoblot. Despite only a 1.4-fold 
A

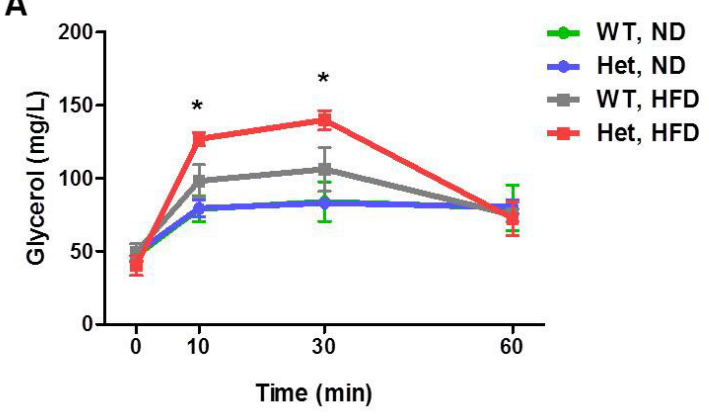

C

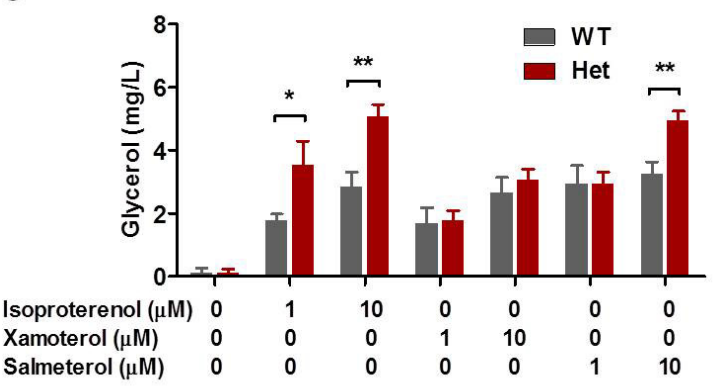

B

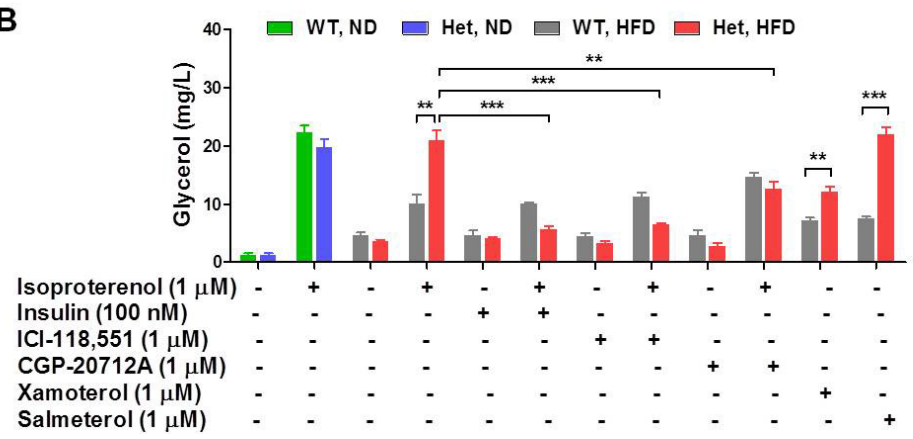

D

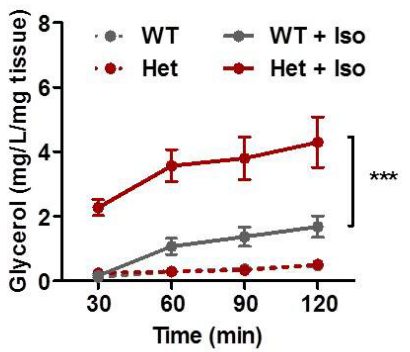

Figure 4.1 Downregulation of NEDD4 Enhances Hormone-stimulated Lipolysis In vivo and Ex vivo.

(A) Serum free glycerol levels in a cohort of 22-week-old, gender-matched wild-type (WT) and $\mathrm{Nedd}^{+/-}$(Het) mice at indicated time after i.p. isoproterenol, $10 \mathrm{mg} / \mathrm{kg}(\mathrm{n}=5)$. (B) Primary adipocytes purified from epididymal fat of WT and Het mice were resuspended in media and incubated with indicated reagents with gentle shaking for $1 \mathrm{hr}$. Insulin, $\beta$-agonists, and $\beta$-antagonists were pre-incubated for $10 \mathrm{~min}$. Glycerol levels in conditioned media were measured $(n=4)$. (C) Primary adipocytes were purified from epididymal fat of aged WT and Het mice and resuspended in media with indicated reagents. Glycerol levels in media were measured after 1-hr incubation $(n=5-6)$. (D) Adipose tissue explants from aged WT and Het mice were resuspended in DMEM supplemented with $0.5 \%$ fatty acid free BSA and shaken at $400 \mathrm{rpm}$ at $37^{\circ} \mathrm{C}$ with or without $1 \mu \mathrm{M}$ isoproterenol for $1 \mathrm{hr}$. Glycerol levels were normalized to the weights of adipose tissue explant $(\mathrm{n}=4)$. Data represent mean $\pm \mathrm{SEM} ;{ }^{*} P<0.05,{ }^{* *} P<0.01$, and $* * * P<0.001$. 
increase in total $\beta_{2}$-AR protein levels (Figure 4.2A), a dramatic 2.4-fold increase in membrane $\beta_{2}$-AR levels was detected (Figure 4.2B). These observations suggest that the enhanced lipolysis associated with NEDD4 reduction is likely due to increased abundance of $\beta_{2}$-AR on the adipocyte plasma membrane. We failed to detect $\beta_{1}$-AR in these experiments, owing to high background with the chosen antibody. Concurrently, we observed a 1.5-fold higher level of IR, but not IGF-1R, on the plasma membrane in $N e d d 4^{+/-}$mice on HFD (Figure 4.2B), consistent with the observations of elevated insulin signaling in WAT.

To confirm these observations in vitro, we employed HEK293 cells with stable ectopic overexpression of human $\beta_{2}$-AR at a medium level. Downregulation of membrane $\beta_{2}$-AR by prolonged isoproterenol incubation was largely rescued by either NEDD4 knockdown or transient overexpression of a dominant negative NEDD4 (a CS mutant lacking E3 ligase activity) (Figure 4.2C).

\subsubsection{Knockdown of NEDD4 Increases Lipolytic but Inhibits Adipogenic Response in 3T3-L1 Adipocytes}

NEDD4 is present at high concentration in 3T3-L1 cells. The 3T3-L1 cell line is one of the most hard-to-transfect cell lines. Therefore, it is technically difficult to completely deplete NEDD4 from 3T3-L1 cells. Using adeno-associated virus (AAV)delivery of specific shRNA targeting Nedd4 mRNA (EGFP-tagged), we were able to downregulate both Nedd4 mRNA and NEDD4 protein levels by $\sim 50 \%$ (Figure 4.3C). Knocking down NEDD4 in 3T3-L1 adipocytes produced a subtle but significant increase in isoproterenol-stimulated lipolysis. These data suggest that the enhanced lipolytic responses observed in HFD-fed or aged $\mathrm{Nedd}^{+/-}$mice are most likely a direct result of NEDD4 downregulation and not a secondary (endocrine) effect from other organs. Since 3T3-L1 adipocytes express predominantly $\beta_{2}$-subtype AR, these data imply that $\beta_{2}$-AR is regulated by NEDD4 in adipocytes (Figure 4.3A).

Another intriguing finding was that knocking down NEDD4 in 3T3-L1 cells by AAV-delivered shRNA greatly impeded the efficiency of the preadipocyte-to-adipocyte conversion (Figure 4.3B). PPAR $\gamma$ is arguably the central regulator of adipogenesis. To examine whether NEDD4 affects PPAR $\gamma$ levels, we measured PPAR $\gamma$ expression in shNEDD4 AAV-infected 3T3-L1 adipocytes. Strikingly, we found a similar degree $(\sim 50 \%)$ of PPAR $\gamma$ downregulation to NEDD4 reduction $(P<0.001)$ (Figure 4.3C), together with a consistent $\sim 12 \%$ reduction in the mRNA levels for aP2 and Cebpa, two PPAR $\gamma$ downstream target genes. Despite the decrease in PPAR $\gamma$ protein, The Pparg mRNA levels were not altered in comparison to those infected with scrambled shRNA AAV (Figure 4.3D). At terminal differentiation, there were still approximately $50 \%$ of the cells expressing EGFP-tagged shRNA viruses (Figure 4.3E).

To examine whether NEDD4 affects PPAR $\gamma$ levels in vivo, we measured PPAR $\gamma$ expression in epididymal WAT. Western blot analysis revealed a trend of reduction in the protein levels of both PPAR $\gamma$ isoforms (but not C/EBP $\alpha$ ) in both HFD-fed and aged 
A

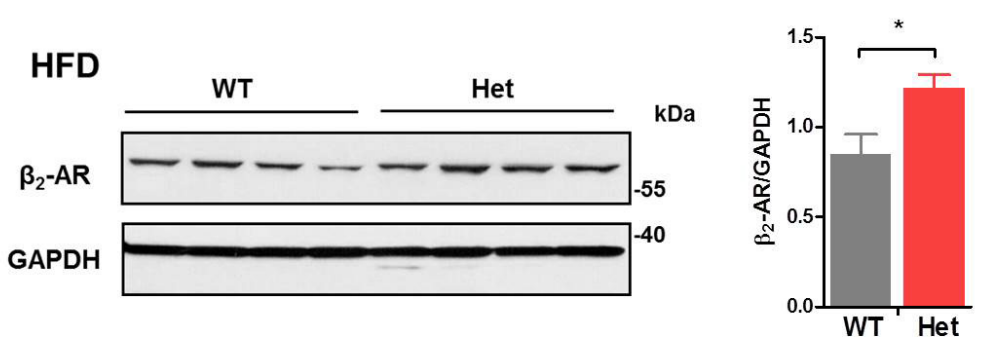

B

HFD

\begin{tabular}{|c|c|c|}
\hline & WT Het & kDa \\
\hline NEDD4 & $-\cdots$ & -130 \\
\hline$\beta_{2}-A R$ & -- & -55 \\
\hline $\operatorname{IR} \beta$ & $\cdots-$ & -100 \\
\hline IGF-1R $\beta$ & $\cdots$ & -100 \\
\hline
\end{tabular}

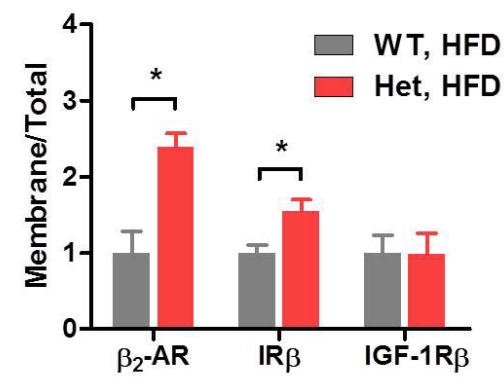

C
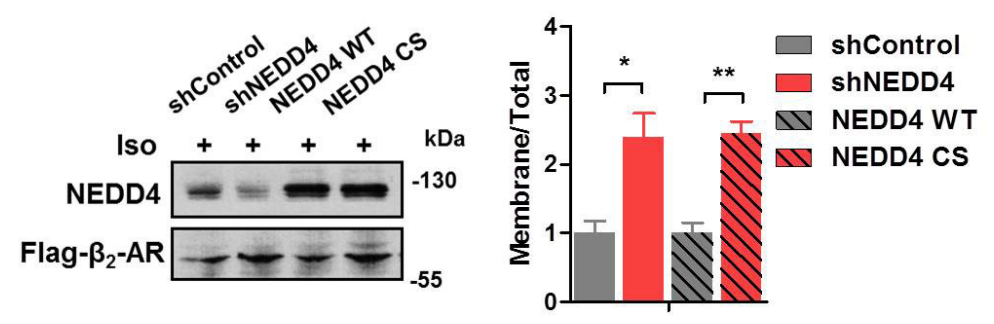

Figure 4.2 Loss of NEDD4 Function Increases $\beta 2-A R$ Protein Concentration at Plasma Membrane.

(A) Representative Western blot for $\beta_{2}$-AR expression in epididymal WAT of wild-type (WT) and Nedd4 ${ }^{+/-}$(Het) mice after HFD (n=4). (B) Representative Western blot for crude membrane fraction from primary adipocytes purified from WT and Het mice after HFD ( $n=3$ ). (C) $\beta_{2}$-AR-Flag overexpressing cells were transfected with control shRNA (shControl), NEDD4 shRNA (shNEDD4), NEDD4 WT, or NEDD4 CS mutant. After 24hr incubation with $10 \mu \mathrm{M}$ isoproterenol, crude membrane fractions were prepared for Western blot. Data represent three independent experiments. Data represent means \pm SEM; * $P<0.05, * * P<0.01$. 
A
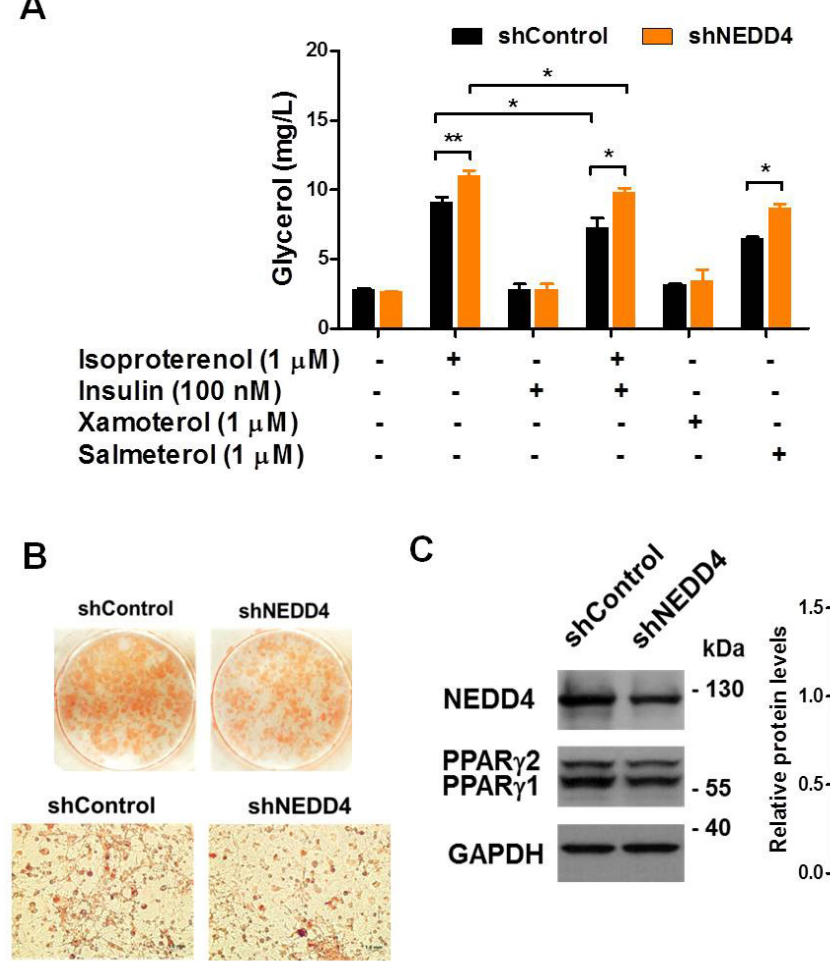

C

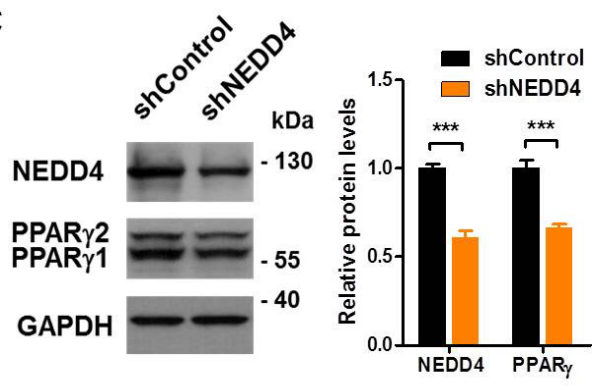

D

$E$
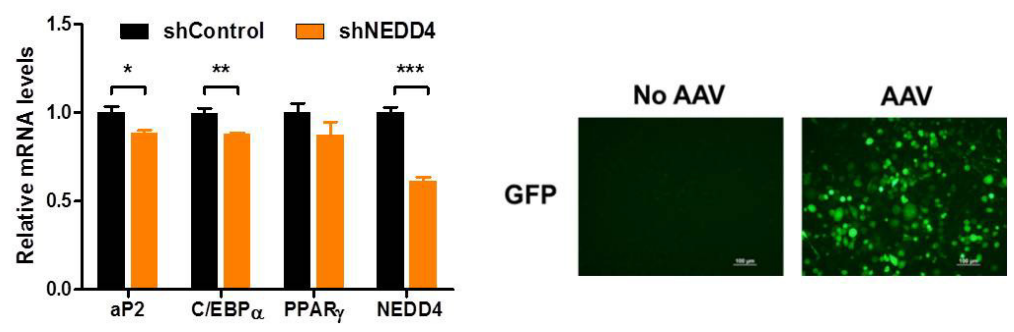

Figure 4.3 Partial Loss of NEDD4 Function Increases Lipolytic but Inhibits Adipogenic Response.

(A) 3T3-L1 adipocytes were infected with AAV virus for expressing shRNA sequences targeting NEDD4 (shNEDD4) or non-targeting shRNA control (shControl), then incubated with indicated reagents for $1 \mathrm{hr}$. Conditioned media were collected and centrifuged to remove cell debris. Glycerol concentrations in the media were measured. Data represent three independent experiments. (B) 3T3-L1 cells were infected with shControl-AAV or shNEDD4-AAV and induced to differentiation as described in Chapter 2. Differentiated adipocytes were stained with oil red $\mathrm{O}$ and photographed. $(\mathrm{C}$ and D) Representative Western blot (C) and qRT-PCR (D) analysis in 3T3-L1 adipocytes infected with indicated virus. Data represent four independent experiments. (E) Differentiated adipocytes were stained with oil red $\mathrm{O}$ and photographed. At terminal differentiation, there were still approximately $50 \%$ of the cells expressing EGFP. Data represent mean $\pm \mathrm{SEM} ; * P<0.05, * * P<0.01$ and $* * * P<0.001$. 
Nedd $4^{+/}$mice (Figure 4.4A and B). The qRT-PCR analysis did not detect significant changes in the mRNA levels of these adipogenic transcription factors (Figure 4.4C).

To our surprise, NEDD4 did not directly modulate PPAR $\gamma$ activity. Based on a PPAR $\gamma$ luciferase reporter assay, PPAR $\gamma$ transactivation by rosiglitazone was not altered by NEDD4 knockdown, NEDD4 overexpression, or overexpression of a mutant NEDD4 (C854S) lacking E3 ligase activity (Figure 4.5). These data indicate that NEDD4 altered PPAR $\gamma$ abundance at the protein level.

\subsubsection{Knockdown of NEDD4 Destabilizes PPAR $\gamma$ Protein}

Next, we tested the role of NEDD4 as a regulator of PPAR $\gamma$ protein. Since NEDD4 and PPAR $\gamma$ expression are positively correlated, we hypothesized that NEDD4 acts as a stabilizer for PPAR $\gamma$ protein. To test this hypothesis, we manipulated NEDD4 expression in HEK293 cells transiently overexpressing PPAR $\gamma$, and blocked the de novo protein synthesis within the cells using cycloheximide. After $6 \mathrm{hr}$ incubation, cells that had reduced NEDD4 expression also had significantly lower PPAR $\gamma$ concentration (Figure 4.6A and C). NEDD4 or NEDD4-CS upregulation in cells protected PPAR $\gamma$ protein from degradation, with most dramatic effect within $4 \mathrm{hr}$ (Figure 4.6B and D), suggesting that the stabilization does not require the enzymatic function of NEDD4.

By analyzing PPAR $\gamma$ sequence, we identified a PPxY motif within PPAR $\gamma$ that could serve as a binding site for NEDD4 (Figure 4.7A). Indeed, NEDD4 and PPAR $\gamma$ could be pulled down together in overexpressed culture system demonstrating that these proteins interact. The same interaction was found in 3T3-L1 adipocytes and mouse WAT (Figure 4.7B-D). To investigate whether the interaction between NEDD4 and PPAR $\gamma$ is altered during adipogenesis, we co-immunoprecipitated these proteins from 3T3-L1 cell lysates collected throughout differentiation. Remarkably, NEDD4-PPAR $\gamma$ interaction was the strongest in preadipocytes, then gradually weakened as differentiation continued

(Figure 4.8). The reciprocal changes observed in the NEDD4-PPAR $\gamma$ interaction and the PPAR $\gamma$ levels during adipogenesis strongly suggest a central role for NEDD4 in adipogenesis which warrants further investigation.

\subsection{Summary}

In this chapter, we studied potential molecular and cellular mechanisms that lead to the "lean" phenotype in Nedd $4^{+/-}$mice. As shown in the previous chapter, these mice had normal energy intake and exhibited similar physical activity to wild-type mice. The transcriptional expression of several key thermogenesis-related genes in BAT in Nedd $4^{+/}$ mice was also undistinguishable from that in wild-type animals, suggesting unaltered thermogenesis.

Due to resource limitation, we did not examine the basal metabolic rates in these mice, therefore, cannot rule out the contribution from basal metabolism. Nonetheless, we 
A
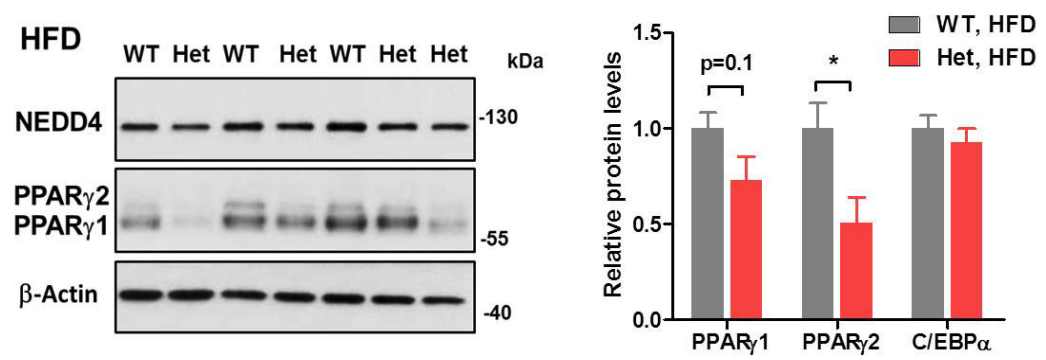

B
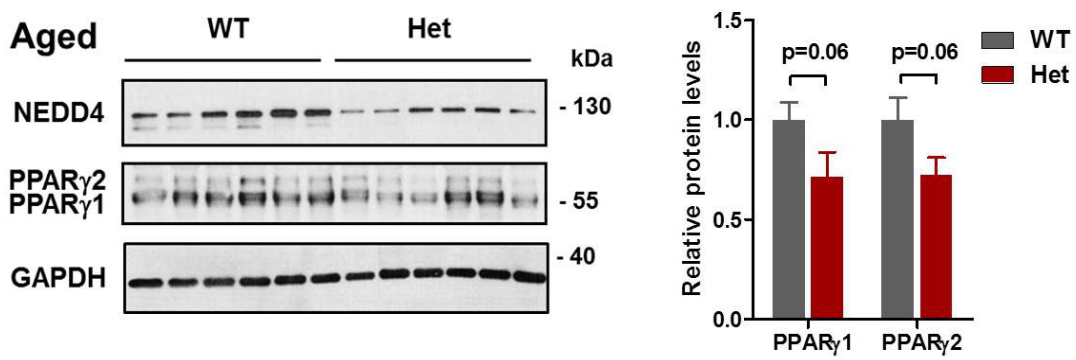

C

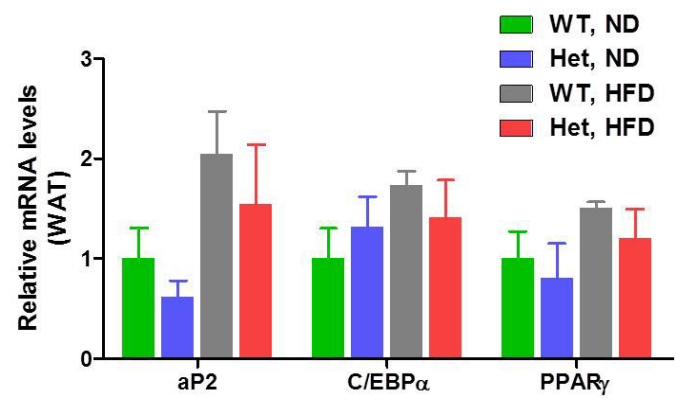

Figure 4.4 Partial Loss of NEDD4 Function Reduces PPAR $\gamma$ Levels in WAT.

(A and B)Western blot of PPAR $\gamma$ protein levels in epididymal fat from gender-matched, HFD-fed, wild-type (WT) and Nedd4 ${ }^{+/-}$(Het) littermates at age 22 weeks (A) and age 18 months (B) (A, n=7-8; B, n=6). (C) Relative transcriptional levels of aP2, C/EBP $\alpha$ and PPAR $\gamma$ in epididymal fat in littermate WT and Het mice on ND or HFD were measured by qRT-PCR (ND, $\mathrm{n}=3$, HFD, $\mathrm{n}=5-6$ ). Data represent mean $\pm \mathrm{SEM} ; * P<0.05$. 


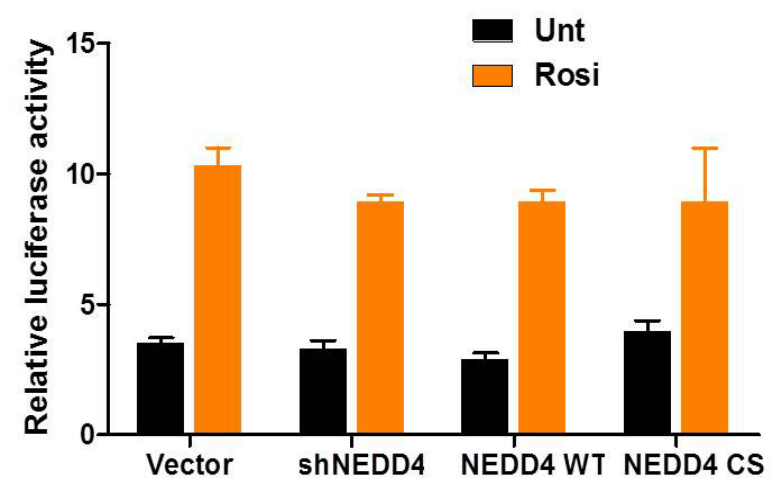

Figure 4.5 NEDD4 Has No Direct Effect on PPAR $\gamma$ Activity.

HEK293 cells were seeded in a 24-well plate and left overnight for attachment. Each well was transiently co-transfected with $250 \mathrm{ng} 3 \times$ PPRE-Luc, $250 \mathrm{ng}$ PPAR $\gamma 2$-Flag, $10 \mathrm{ng}$ Renilla luciferase control reporter vector pRL-SV40 and 250 ng plasmids indicated under each column. After $32 \mathrm{hr}$ of transfection, cells were treated with or without rosiglitazone $(10 \mu \mathrm{M})$ for $16 \mathrm{hr}$ prior to luciferase assay. Luciferase activity was normalized to Renilla activity as a control for transfection efficiency. Data represent three independent experiments. 
A

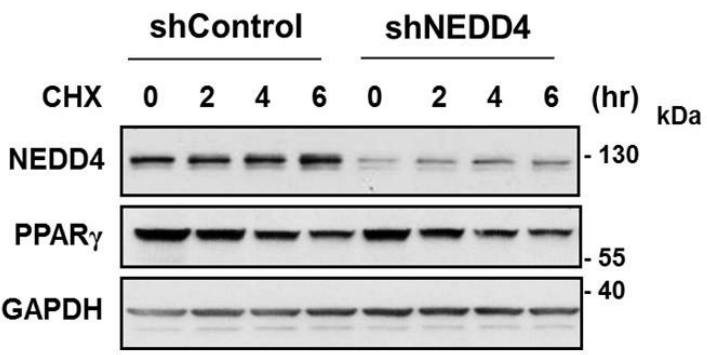

B

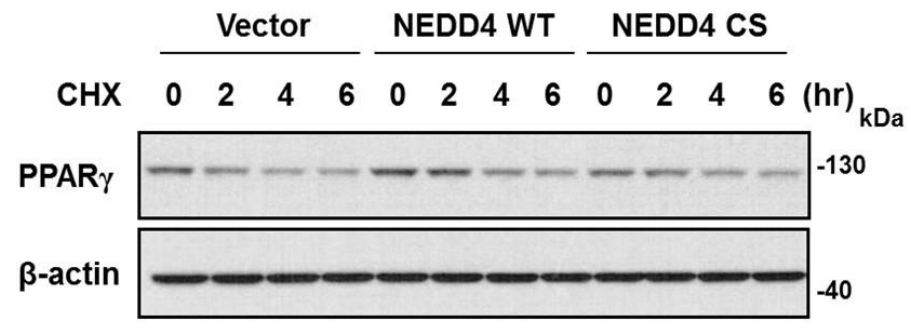

C
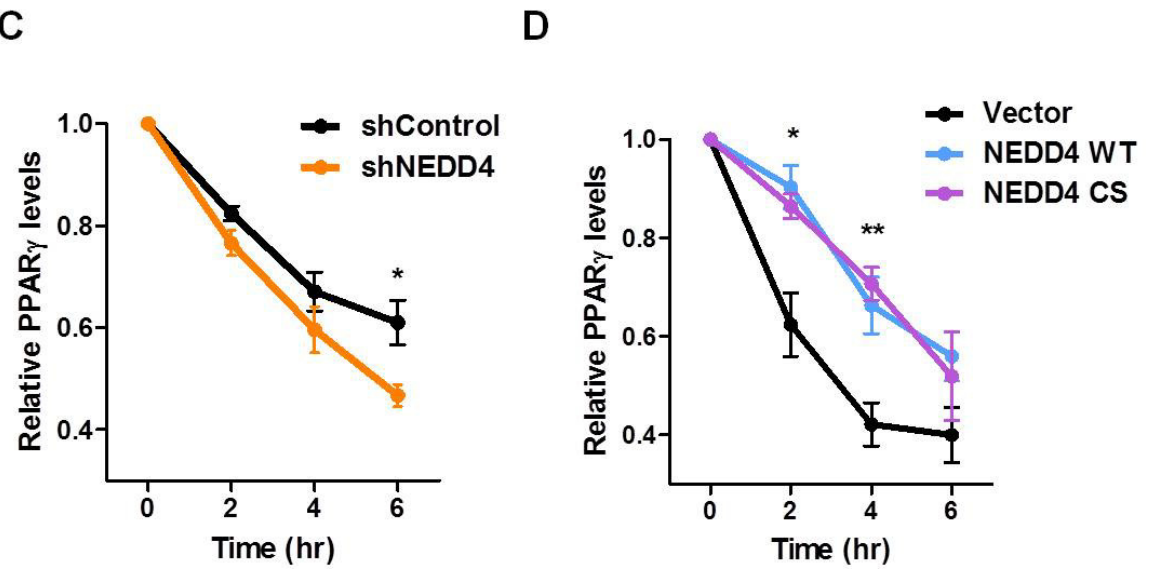

Figure 4.6 NEDD4 Stabilizes PPAR $\gamma$ Protein Expression In vitro.

HEK293 cells were seeded in a 6-well plate and left overnight for attachment. Each well was transiently co-transfected with $1 \mu \mathrm{g}$ PPAR $\gamma$-Flag and $1 \mu \mathrm{g}$ indicated plasmids. After $48 \mathrm{hr}$, cells were incubated with $20 \mu \mathrm{M}$ of cycloheximide (CHX) for 2, 4, and $6 \mathrm{hr}$. (A and B) Representative Western-blot image of PPAR $\gamma$ protein levels during $\mathrm{CHX}$-chase experiment. (A) NEDD4 knockdown by shRNA. (B) NEDD4 or NEDD4 CS mutant overexpression. (C) Quantification data for the effect of NEDD4 knockdown on PPAR $\gamma$ protein levels. (D) Quantification data for the effect of NEDD4 or NEDD4 CS mutant overexpression on PPAR $\gamma$ protein levels. Data represent three independent experiments. Data represent mean $\pm \mathrm{SEM} ; * P<0.05,{ }^{* *} P<0.01$; n.s., no significance. 
A

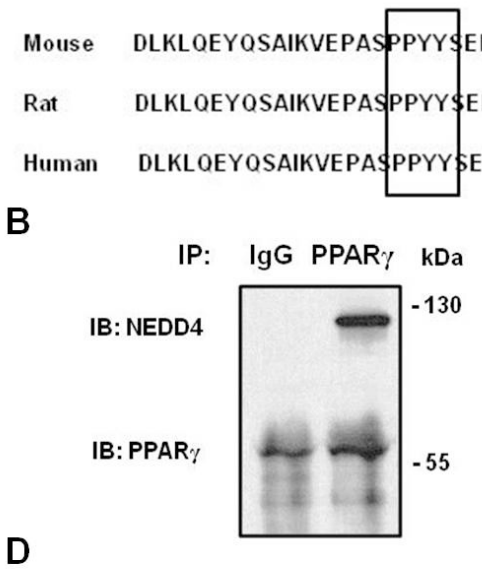

C

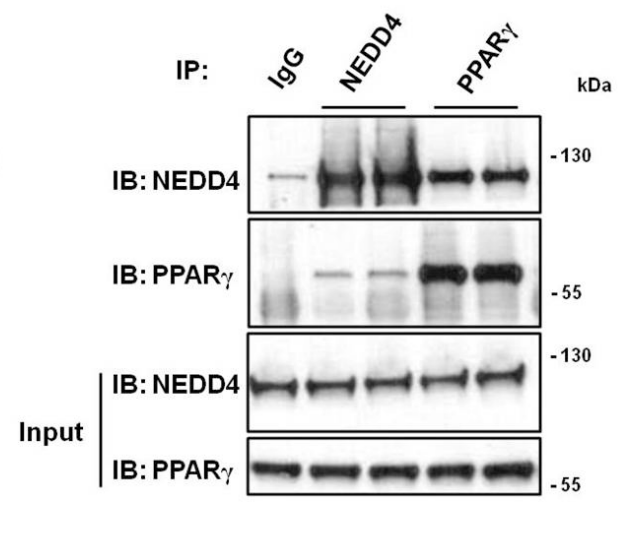

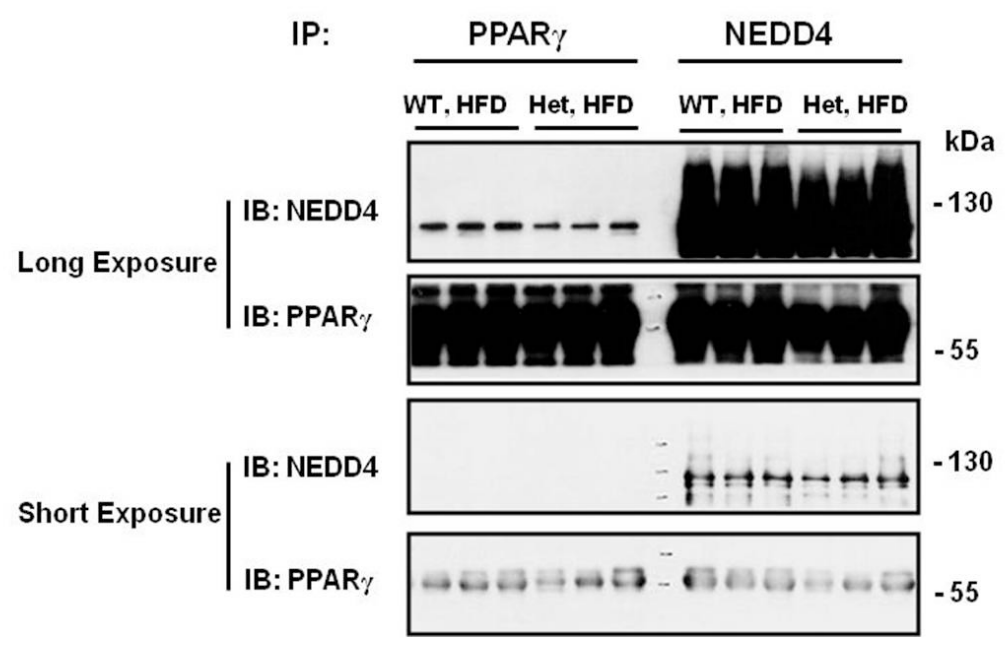

Figure 4.7 NEDD4 Interact with PPAR $\gamma$.

(A) Sequence alignment of PPxY motif within PPAR $\gamma$ in various species. (B) Coimmunoprecipitation of PPAR $\gamma$ in 3T3-L1 adipocytes with $1 \mu \mathrm{g}$ of anti-PPAR $\gamma$ antibody (E8, Santa Cruz) and $500 \mu \mathrm{g}$ of extract. Co-IP using normal mouse IgG served as negative control. (B) Co-immunoprecipitation of NEDD4 or PPAR $\gamma$ in HEK293 cells transiently overexpressing plasmids containing NEDD4 and PPAR $\gamma 2$ with respective antibodies. Lysates pulled down with anti-IgG served as negative control. (D) Coimmunoprecipitation of NEDD4 or PPAR $\gamma$ in $500 \mu \mathrm{g}$ protein lysates of epididymal WAT dissected from wild-type (WT) and $\mathrm{Nedd}^{+/-}$(Het) mice. 
A
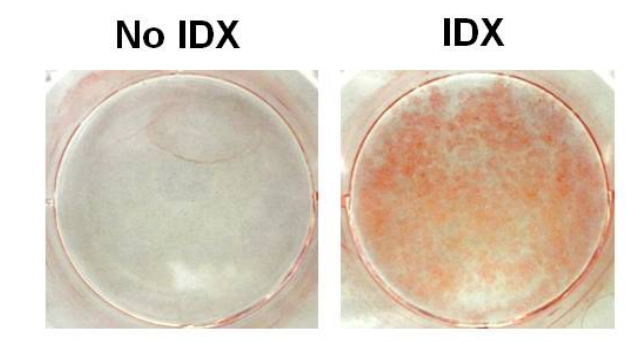

B

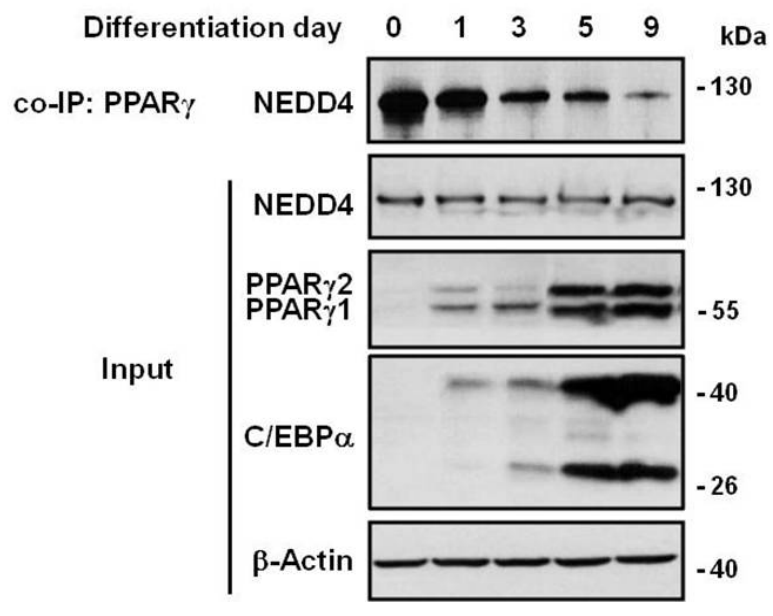

Figure 4.8 NEDD4-PPAR $\gamma$ Interaction Alteration during Adipocyte Conversion in 3T3-L1 Cells.

(A) Oil red O staining on 3T3-L1 cells incubated with or without IDX cocktail $(0.5 \mathrm{mM}$ IBMX, $1 \mu \mathrm{M}$ dexamethasone, and $1.5 \mu \mathrm{g} / \mathrm{mL}$ insulin). Picture was taken at 7 days after IDX treatment. (B) After indicated time of differentiation, 3T3-L1 cells were harvested, immunoprecipitated with an antibody against PPAR $\gamma$ (Santa Cruz E8), followed by NEDD4 Western blot. Whole cell lysates (input) were immunoblotted for indicated proteins. Co-IP antibody: $1 \mu \mathrm{g}$; extract: $500 \mu \mathrm{g}$; input: $20 \mu \mathrm{g}$. Data represent two independent experiments. 
provide substantial evidence from in vivo and in vitro experiments implying that NEDD4 is a regulator of two important pathways in lipid metabolism, i.e. lipolysis and adipogenesis, via modulating $\beta_{2}-\mathrm{AR}$ and PPAR $\gamma$ functions. Isoproterenol stimulated more glycerol release in $\mathrm{Nedd} 4^{+/-}$mice and isolated $\mathrm{Nedd} 4^{+/-}$adipocytes, suggesting enhanced $\beta$-adrenergic signaling. Data from the treatments of different $\beta$ agonists or co-incubation of isoproterenol with specific $\beta$ antagonists suggested that $\beta_{2}$-AR primarily mediated the enhanced lipolytic response by NEDD4 downregulation. Concurrently, Western blot analysis of total and membranous $\beta_{2}$-ARs in adipose tissue revealed higher expression of the receptor, especially membranous receptor in $N e d d 4^{+/-}$mice. NEDD4 knockdown or overexpressing a dominant negative NEDD4 increased $\beta_{2}$-AR levels in crude membrane fractions from HEK293 cells stably expressing Flag- $\beta_{2}-A R$. These data support a previously identified NEDD4's role as an E3 ligase for $\beta_{2}$-AR protein degradation (Shenoy et al., 2008).

Using 3T3-L1 cells as a model, we found that downregulation of NEDD4 in 3T3L1 preadipocytes inhibited their adipogenic response by the IDX cocktail. The protein but not mRNA level of PPAR $\gamma$, a key adipogenic transcription factor, was also reduced in NEDD4 knockdown 3T3-L1 cells. We confirmed this positive correlation between NEDD4 and PPAR $\gamma$ in vivo in WAT from both HFD-fed and aged mice. Overexpression of NEDD4 stabilized ectopically expressed PPAR $\gamma$, while NEDD4 knockdown destabilized it. This stabilization effect appeared to be independent of NEDD4's ubiquitin ligase function, as overexpression of a dominant negative form of NEDD4 could stabilize PPAR $\gamma$ to similar extend as wild type NEDD4. Moreover, NEDD4 and PPAR $\gamma$ could be co-immunoprecipitated as a complex; their interaction was strongest in preadipocytes and gradually attenuated during adipocyte conversion further indicating an important role of NEDD4 in adipogenesis.

In summary, the delayed development of HFDIO in Nedd4 ${ }^{+/-}$mice can be explained at least through the NEDD4-mediated regulation of lipolysis and adipogenesis pathways. These findings again support a novel role of NEDD4 in the molecular pathophysiology of obesity. 


\section{CHAPTER 5. NEUROBEHAVIORAL ANALYSES}

\subsection{Introduction}

Ubiquitin-mediated proteolysis, being undeniably important for most intracellular processes, is increasingly recognized as a critical mechanism in the regulation of neuronal development and function in the brain. In Chapters 3 and 4, we have studied NEDD4's role in metabolic function in the context of obesity. In the current chapter, we will extend our investigation to NEDD4's neuronal functionality.

A unique morphological feature of neurons is that they possess axons and dendrites (collectively known as neurites), which are projections of neurons that serve to transmit electrical/electrochemical signals. Neurons are connected to each other with their neurites to form neural networks. Typically, a neuron propagates electrical signals through its axon. At the axon terminal, electrical signals trigger release of neurotransmitters which can be received by dendrites from other neurons. The axon terminal, the receiving surface of dendrite, and the cleft in between constitute a synapse, whose plasticity (i.e. synaptic plasticity) plays important role in learning and memory.

NEDD4 is highly expressed in mammalian neurons. Through modulation on its neuronal substrates, it has been implicated in at least four aspects of neuronal physiology

- that is neurite outgrowth, neuron survival, ion channel functions, and neurotransmitter signaling. Two studies in 2010 demonstrated an integral role of NEDD4 in the growth and branching of neurites: Drinjakovic et al. reported that disrupting NEDD4 function severely inhibits the terminal branching of retinal ganglion cell axons by downregulating PTEN (Kawabe et al., 2010). Similarly, using both conventional and conditional NEDD4 knockout mice, Kawabe et al. identified NEDD4 as an important player in promoting dendrite formation through inhibiting Rap2A function (Kawabe et al., 2010). In addition, NEDD4 was involved in regulating neuronal survival via targeting PTEN for degradation in neurons, as demonstrated previously in our laboratory (Kwak et al., 2010). Also, NEDD4 was able to decrease whole cell $\mathrm{Ca}(\mathrm{v})$ currents by downregulating $\mathrm{Ca}(\mathrm{v})$ calcium channels (Rougier et al., 2011) and to mediate agonist-dependent ubiquitination and degradation of GluA1-containing $\alpha$-amino-3-hydroxy-5-methyl-4-isoxazolepropionic acid receptor (AMPAR) (Schwarz et al., 2010). Most of these studies were conducted either in vitro or at the embryonic stage, raising an interesting question about the NEDD4's neuronal physiological roles in postnatal life.

The sporadic Alzheimer's disease (AD), the most common form of dementia, has been increasingly linked to T2D. Some even term AD as "type 3" diabetes, as many observations in $\mathrm{AD}$ brains reflect similarities in $\mathrm{T} 2 \mathrm{D}$, such as insulin resistance and reduced glucose utilization in the brain (Liao and $\mathrm{Xu}, 2009$; Steen et al., 2005). In addition, several lines of evidence indicate that boosting insulin signaling in the brain via intranasal insulin administration improves cognition in AD patients(Benedict et al., 2004; Craft et al., 2012; Dhamoon et al., 2009; Reger et al., 2008). One of the research interests in our laboratories is to identify the molecular links between these two types of disease. 
Previous studies from our laboratory and others(Cao et al., 2008; Drinjakovic et al., 2010; Kawabe et al., 2010; Kwak et al., 2012) demonstrated that NEDD4 is involved in insulin/IGF-1 signaling, neurite development, and is likely implicated in AD progression, as the expression levels of NEDD4 are increased in postmortem AD brains. Here, we investigated whether heterozygous deletion of Nedd4 in mice can alter CNS metabolism (insulin signaling, in particular) which may lead to neurobehavioral alteration in vivo.

\subsection{Results}

\subsubsection{Nedd4-haploinsufficient Mice Have Improved Fear-associated Memory}

Fear conditioning is an experimental paradigm to study fear associated memory. Normally, animals are challenged with electric shocks paired with a certain context (e.g. a room) and a certain cue (e.g. a tone). After a period of time, the fear response of animals (freezing) is tested when re-exposed to the same context or cue. This is a form of learning and memory mainly dependent upon a brain region called amygdala. But the contextual fear conditioning is believed to involve also hippocampus (Phillips and LeDoux, 1992). When exposing to foot shock, $\mathrm{Nedd}^{+/-}$and control mice displayed similar immediate freezing response, indicating that there is no difference in their threshold for pain (Figure 5.1A). After $24 \mathrm{hr}$, we placed these mice individually in boxes with changed context (decoration and bedding), played the same cue again as on day 1 , and recorded their freezing time. The result showed no significant difference in cued fear conditioning (Figure 5.1B). Another $3 \mathrm{hr}$ after tone test, we placed these mice back in exactly the same box as on the training day. The $N e d d 4^{+/-}$mice showed higher freezing response than their wild-type littermates (1.4-fold, $P<0.01)$, suggesting better fearassociated memory in the Nedd $4^{+/}$mice (Figure 5.1C).

\subsubsection{Nedd4-haploinsufficient Mice Display More Spontaneous Alternation}

The cross maze is a behavioral test commonly used for assessing animals' spatial learning and memory, which is believed to depend on the function of hippocampus (Lalonde, 2002). Rodents possess a strong tendency on arm alternation, which is they

prefer to explore new arms. The "smart" mice should remember which maze arm was last visited. Therefore, recording the orders of their entries is used as an index of the spatial reference memory. We placed a cohort of $N e d d 4^{+/-}$and wild-type mice individually in a standard cross maze and recorded their order of arm entries. At the end of the test, the total number of arm entries and alternation were analyzed. Interestingly, the $\mathrm{Nedd} 4^{+/-}$ group had significantly higher percentage of alternation $(36.0 \pm 2.8 \%$ vs. $27.4 \pm 1.8 \%$, $P<0.01$ ) than wild-type group (Figure 5.2), indicating better spatial working memory in $\mathrm{Nedd} 4^{+/-}$mice. 

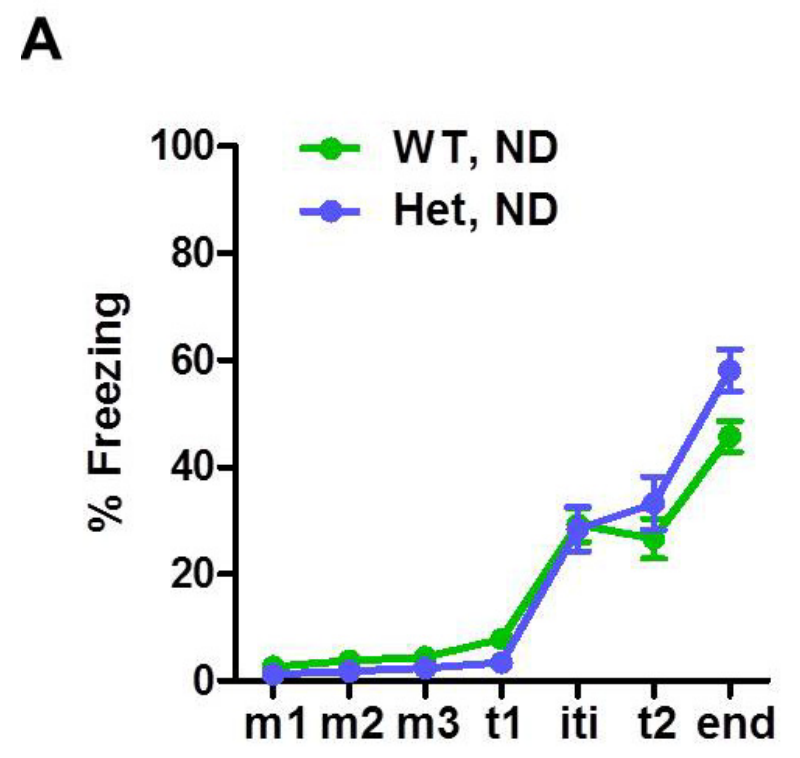

B
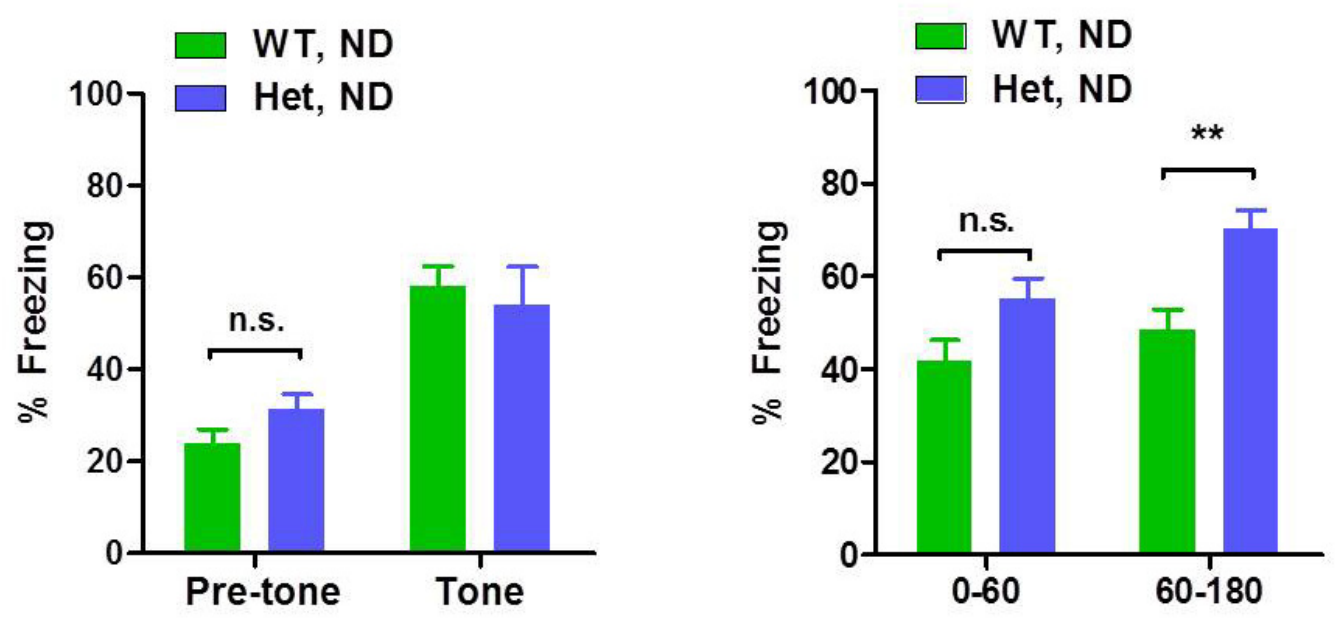

Figure 5.1 Improved Fear-associated Memory in Nedd4-haploinsufficient Mice. (A) Immediate freezing score during the first day training ( $n=23-46$ per group). Time intervals for the fear conditioning training on day $1 . \mathrm{m} 1$ : minute 1, 0-60 s; $\mathrm{m} 2$ : minute 2, 60-120 sec; m3: minute 3, 120-180 sec; tone 1: 180-200 sec; iti: inter-tone interval, 200-260 sec; tone 2: 260-280 sec; end: 280-340 sec. (B and C) Freezing score during retrieval tone test (B) or contextual test (C) on day 2 ( $n=23-46$ per group). Time intervals for the tone retrieval test on day 2: pre-tone: 0-180 sec; tone: 180-200 sec. Time interval for the context fear retrieval test on day 2: context: $60-180 \mathrm{sec}$. Data represent mean \pm SEM; $* * P<0.01$; n.s., no significance. 
A

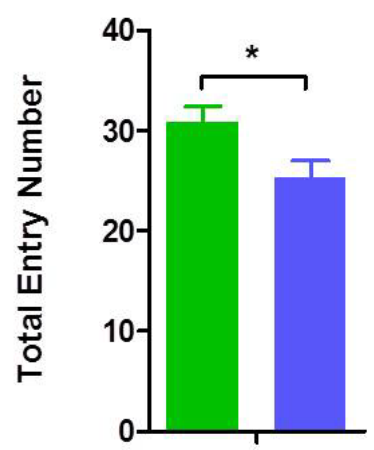

B

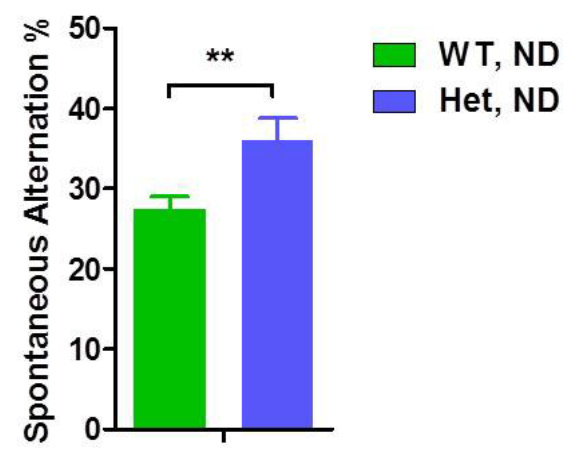

Figure 5.2 Improved Learning and Memory in Cross Maze Test in Nedd4haploinsufficient Mice.

(A) Total number of arm entries in Nedd $4^{+/}$(Het) and wild-type (WT) mice ( $\mathrm{n}=14-23$ per group). (B) Ratios of spontaneous alternation versus total entry number ( $\mathrm{n}=14-23$ per group). Data represent mean $\pm \mathrm{SEM} ;{ }^{*} P<0.05, * * P<0.01$. 


\subsubsection{Nedd4-haploinsufficient Mice Display Impaired Water Maze Performance}

Fear conditioning and cross maze indicate different forms of memory (associative and working), but both tests can sometimes be compromised by anxiety/depression. In order to confirm phenotype of improved learning and memory in Nedd $4^{+-}$mice, we performed a third behavioral test, the Morris water maze test, which has become the "gold standard" for assessing hippocampus-dependent spatial learning and memory. To our surprise, the $\mathrm{Ne}_{\mathrm{d}} 4^{+/}$mice displayed severely impaired learning and memory in water maze task. While the latency in wild-type group started from $\sim 36 \mathrm{~s}$ and dropped to $\sim 12 \mathrm{~s}$ at the end of the training, the average latency in $\mathrm{Nedd} 4^{+/-}$mice remained above 30 $\mathrm{sec}$ at day 10 (Figure 5.3A). In the probe test where the hidden platform was removed and mice were allowed to swim in the pool freely for $60 \mathrm{~s}$, unlike the wild-type mice that spent most of the time swimming around the site of hidden platform, $\mathrm{Nedd} 4^{+/-}$mice swam across four platforms randomly (Figure 5.3B). These mice took much longer time in finding the hidden platform, suggesting that they had impaired spatial learning and memory abilities. Brain insulin signaling has been implicated in spatial memory processing (Zhao et al., 1999). To examine the brain insulin signaling in $\mathrm{Nedd}^{+/-}$mice, we dissected cortical tissue from P0 mouse brain and stimulated the tissue with insulin. Interestingly, we found that the insulin signaling in cortical tissue appeared to be attenuated in $\mathrm{Nedd}^{+/-}$mice in a dose- and time-dependent manner, especially within 15 min of insulin administration (Figure 5.4).

\subsubsection{Nedd4-haploinsufficient Mice Display Anxiety-like Behavior}

When testing the physical activity of Nedd $4^{+/-}$mice in an open field test, we found that although the total activity and distance traveled were not distinguishable between $\mathrm{Nedd} 4^{+/-}$and wild-type mice, Nedd $4^{+/-}$mice spent significantly less time in the center of the field (Figure 5.5), This indicates anxiety-like behavior as mice prefer to stay or travel close to the side of the box. To confirm this observation, we tested these mice in a light/dark box test, which is widely used in the assessment of anxiety. Interestingly, Nedd $4^{+/}$mice made fewer transitions and tended to stay in the dark compartment, suggesting higher anxiety levels in Nedd $4^{+/-}$mice than controls (Figure 5.6).

\subsection{Summary}

In the current chapter, we studied the in vivo neuronal behavioral outcome using Nedd $4^{+-}$mice. We found that the partial loss of NEDD4 function is sufficient to cause several neurobehavioral alterations using different experimental paradigms, including fear conditioning, cross maze, Morris water maze, open field and light/dark box test. The seemingly paradoxical observations are that $\mathrm{Ne} d d 4^{+/-}$mice performed in opposing trends in different behavioral paradigms. However, these behavioral tests index distinct working memory. For example, the contextual fear conditioning is a form of fear-associated memory which involved functions of amygdala and hippocampus. Although cross maze and Morris water maze are both tests for assessing rodents' working memory, they are 
A

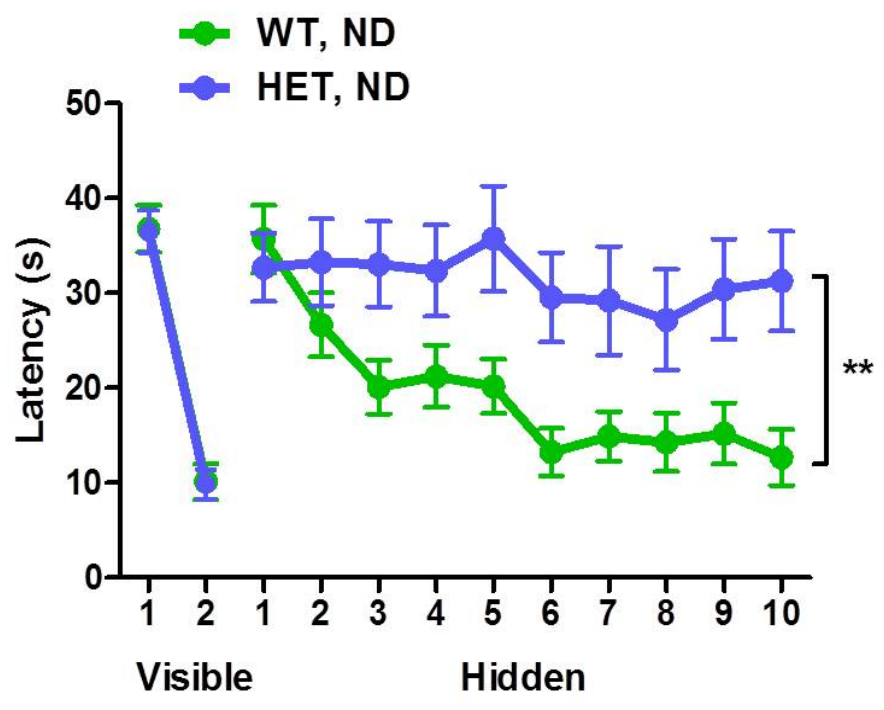

B
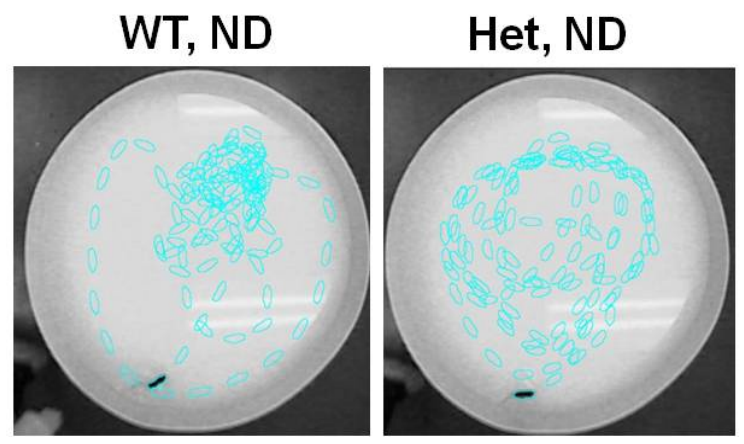

Figure 5.3 Impaired Learning and Memory in Morris Water Maze Test in Nedd4haploinsufficient Mice.

Mice were 2 months old at the beginning of the test. The apparatus is a circular pool filled with RT water made opaque with non-toxic paint. An escape platform was submerged $0.5 \mathrm{~cm}$ below the surface of the water (up-right), to allow the mice to escape from swimming. (A) Latency to find the target platform in the Morris water maze. Both groups had identical swim speed as indicated in the visible section. Nedd $4^{+/-}$(Het) mice spent more time in finding the hidden platform compared to their littermate wild-type (WT) mice from day 3 to day 10 ( $n=14-23$ per group). (B) Representative pictures of swim path in maze during probe trial. Data represent mean $\pm \mathrm{SEM} ; * * P<0.01$. 
A
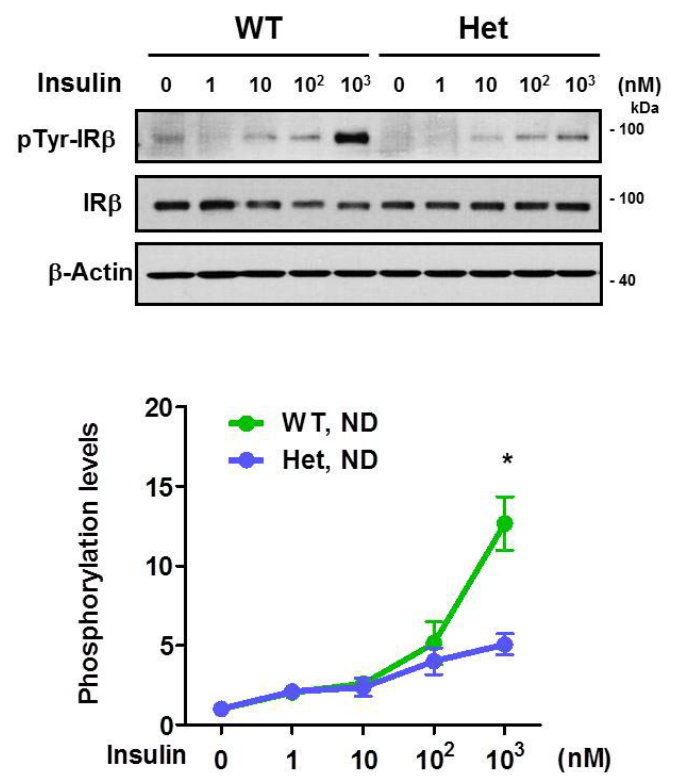

B
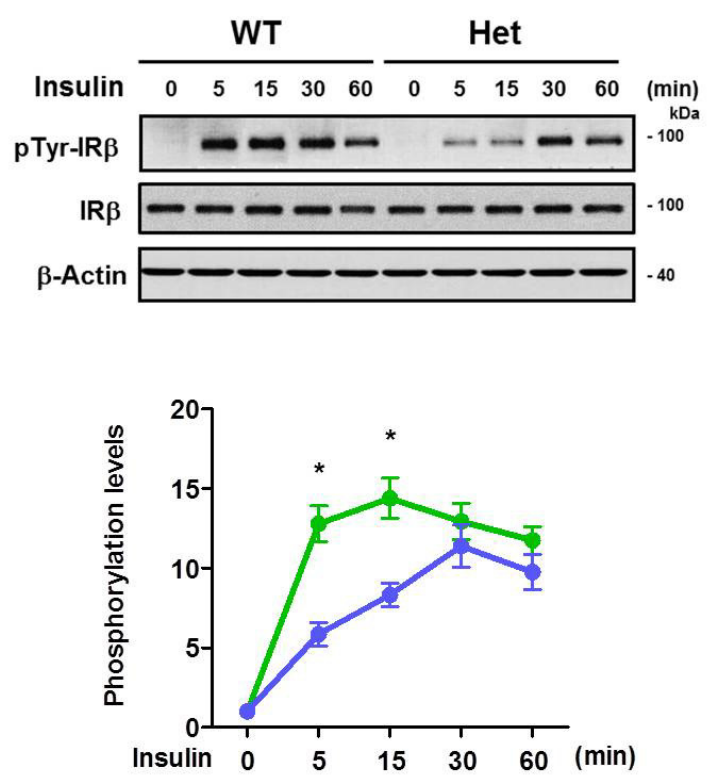

Figure 5.4 Partial Loss of NEDD4 Reduces Insulin-stimulated IR Autophosphorylation.

Dose-response and time-course studies of IR $\beta$ phosphorylation levels in cortex in littermate wild-type (WT) and $N e d d 4^{+/-}$(Het) P0 neonatal mice. (A) The cortical tissue was incubated with different doses of insulin for $15 \mathrm{~min}$. (B) The cortical tissue was incubated with $1 \mu \mathrm{M}$ insulin for indicated times. The bottom panels are protein quantification results. Data represent three independent experiments. All data are mean \pm SEM; $* P<0.05$. 
A

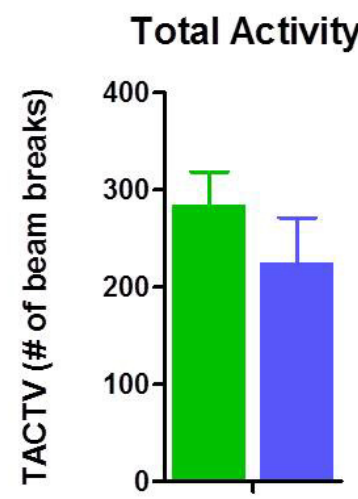

B

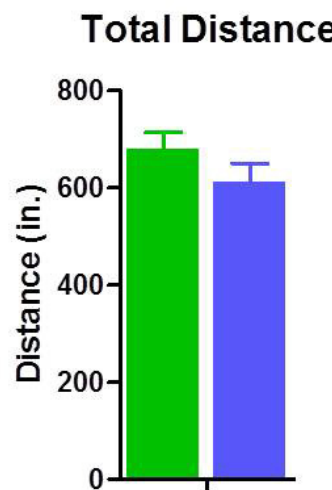

C

Time in Center

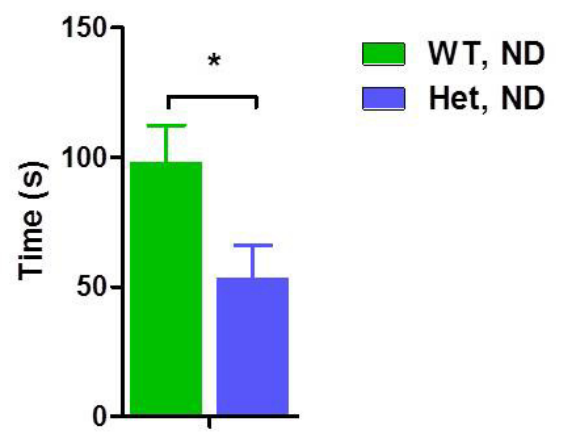

Figure 5.5 Locomotor Activity Test Indicates Anxiety-like Behavior in Nedd4haploinsufficient Mice.

Mouse was placed in an open-field box, equipped with infrared beams that automatically measures total locomotor activity by beam-breaks and with a camera on top that measures the distance the mouse travelled. Mouse was allowed to explore freely in a 10min session. (A) Total number of beam breaks indicates total activity. (B) Total distance traveled in inches. (C) Nedd $4^{+/}$(Het) mice spent less time in center than the wild-type (WT) mice, indicating increased anxiety levels (ND $n=14-23$ per group, HFD $n=8-10$ per group). Data represent mean $\pm \mathrm{SEM} ; * P<0.05$. 
A

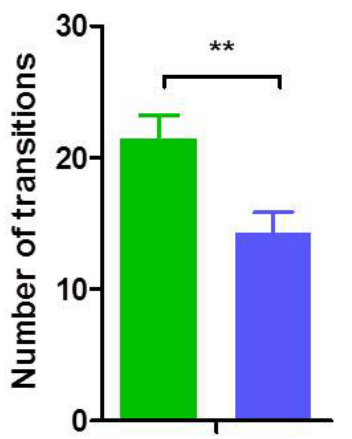

D

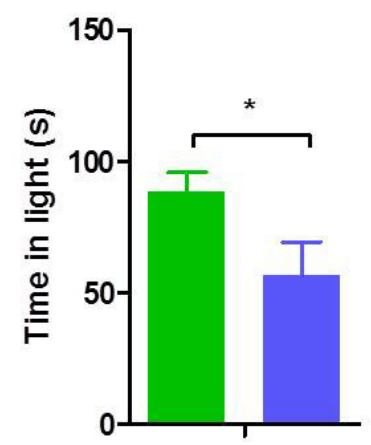

B
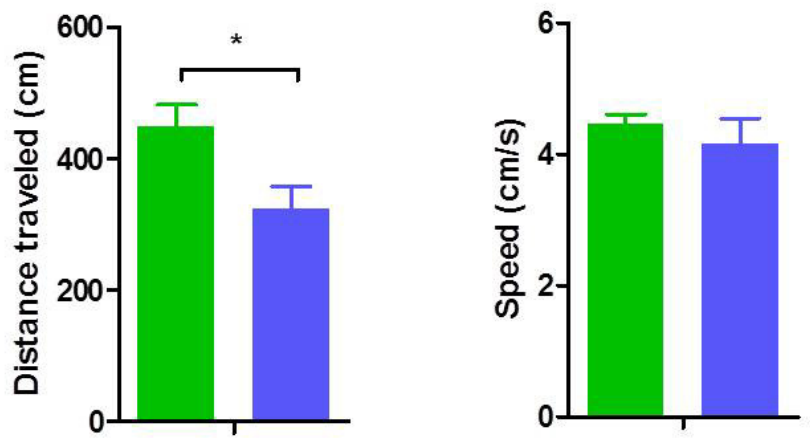

$\mathbf{E}$

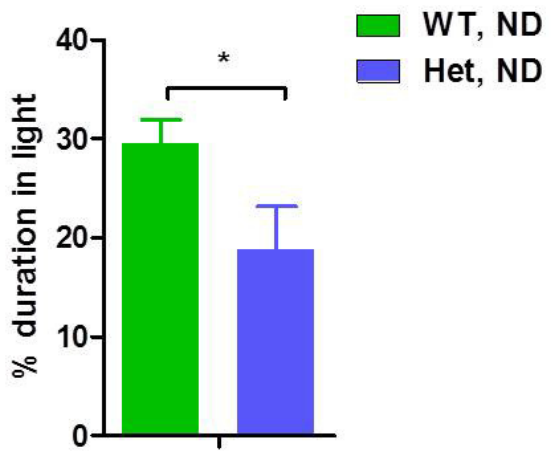

Figure 5.6 Light/dark Box Test Indicates Anxiety-like Behavior in Nedd4haploinsufficient Mice.

Anxiety responses are assessed in a chamber segregated into opaque (dark) and nonopaque (light) compartments. Camera recorded the movement of the mice between compartments. (A) Nedd $4^{+/}$(Het) mice had less number of transitions between light and dark compartments than the wild-type (WT) mice. (B) Het mice traveled shorter distance in the light compartment than the WT mice. (C) Moving speeds were identical between two groups. (D) Het mice spent less time in the light compartment. (E) Percentage of time spent in light compartment was less in Het group. These data indicate that Het mice have increased anxiety levels, as mice have a preference for darkness ( $\mathrm{n}=14-23$ per group). Data represent mean $\pm \mathrm{SEM} ;{ }^{*} P<0.05,{ }^{* *} P<0.01$. 
different in that the prior one is for assessing the short-term memory, while the latter one is for the long-term memory. In Chapter 3, we have shown that NEDD4 regulates insulin signaling in peripheral insulin sensitive tissues under basal condition (ND feeding). Here, we show that the insulin signaling in cerebral cortex in neonatal mice was also

diminished. The impaired long-term spatial working memory of $\mathrm{Nedd} 4^{+-}$mice might be a result of their attenuated insulin signaling in the brain, as central insulin actions are known to have modulatory roles in long-term memory consolidation (Dou et al., 2005). This hypothesis awaits validation in future investigation. 


\section{CHAPTER 6. DISCUSSION}

NEDD4 is the prototypical protein in a family of E3 ligase which has a conserved role in mediating ubiquitin-dependent endocytosis and/or degradation of plasma membrane proteins. In Chapters 3 and 4, we reported that $N e d d 4^{+/-}$mice, despite being moderately insulin resistant, were protected against diet-induced obesity and hepatic steatosis. This provides the first evidence for the involvement of NEDD4 in metabolic regulation. Further, we showed that NEDD4 regulates $\beta$-adrenergic-mediated lipolytic response in vivo and ex vivo. This is consistent to a previous in vitro study of NEDD4- $\beta_{2}-$ adrenergic receptor regulation by Shenoy et al. (Shenoy et al., 2008). In addition, $\mathrm{Nedd} 4^{+/-}$mice under obesogenic (HFD or aged) conditions were accompanied with repressed expression of PPAR $\gamma$ in white adipose tissue. NEDD4 interacted robustly with PPAR $\gamma$ in vitro and in vivo by co-immunoprecipitation. Downregulation of NEDD4 by shRNA-targeting strategy in 3T3-L1 preadipocytes impaired the adipogenic response, resulting in decreased PPAR $\gamma$ protein levels. Thus, our study has identified, for the first time, a positive regulation between NEDD4 and PPAR $\gamma$ in adipocytes. These findings are significant since adipose tissue mass is directly related to the risk of progression from insulin resistance to T2D (Magre et al., 2001). In Chapter 5, we provided preliminary data on several neurobehavioral phenotypes of $\mathrm{Nedd} 4^{+/-}$mice, including altered learning and memory abilities and anxiety levels; we also found reduced insulin signaling in cortical neurons in neonatal $\mathrm{Nedd4^{+/ }}$ mice, indicating an integral role of NEDD4 in maintaining normal neuronal insulin sensitivity and brain function. In summary, our studies provide solid evidence for the involvement of NEDD4 in the regulation of both peripheral metabolism and CNS functions. In this chapter, we attempt to place our studies in context of other published work. We will also discuss the pitfalls of our experiments and implication of the data for future studies.

\subsection{Pathophysiological Roles of NEDD4 in Obesity}

There are a variety of animal models used for obesity research. The most commonly used are the following four categories: genetically derived (ob/ob mice, $d b / d b$ mice and Zucker rats), diet-induced (rodents fed a HFD containing 32-60\% of calories from fat), surgically (ventromedial hypothalamic-lesioned rodents) and chemically (gold thioglucose-treated mice) induced obesity (Lutz and Woods, 2012). Here, we chose to use a specific HFD containing $60 \%$ of calories from fat (mostly lard), which induces rapid weight gain and fat accumulation in several strains of mice. One of these quick responders is the $\mathrm{C} 57 \mathrm{BL} / 6 \mathrm{~J}$ strain of mice. A HFD with $60 \%$ of fat can trigger weight gain and glucose intolerance in $\mathrm{C} 57 \mathrm{BL} / 6 \mathrm{~J}$ mice as early as 1 week and 8 weeks after feeding, respectively (Lin et al., 2000). Obesity, T2D and metabolic syndrome are multifactorial and polygenic in their origins. Therefore HFD feeding strategy is advantageous over monogenically mutated rodent models. Nonetheless, HFDs with various concentrations of fat induce different degrees of obesity in mice. The extreme density of fat in diet $(>60 \mathrm{kcal} \%)$ will result in greatest degree of obesity, which, however, 
is questioned by its physiological relevance (Buettner et al., 2007), as no human food contains such high calories.

By feeding the $\mathrm{Nedd}^{+/-}$and wild-type mice $60 \mathrm{kcal} \%$ fat HFD, and following the body weight changes in these mice for 9 months, we observed that: HFD induced significant weight gain in wild-type male mice after 1 week, while an obvious increase in body weight in $N e d d 4^{+/-}$male mice was observed at 4 weeks after feeding; there was a $30 \%$ decline in the average weight gain in $N e d d 4^{+/}$group of both genders at the end point of the study, though the female mice gained weight more slowly than male mice. Body weight difference between ND-fed $\mathrm{Nedd} 4^{+/}$and wild-type mice revealed significance with two-tailed student's $t$ test, but not with two-way ANOVA with Bonferroni posttest correction when comparing between HFD and ND groups with both genotypes, suggesting that when diet was considered as a factor in addition to genotype, body weights between ND-fed $\mathrm{Nedd} 4^{+/-}$and wild-type mice were not considered significantly different.

\subsubsection{The Energy Balance in Nedd4-haploinsufficient Mice}

In humans, dairy fat through the gastrointestinal tract is transformed to chylomicrons and lipoproteins, a portion of which is metabolized and utilized as energy supply and the rest is stored in adipose tissue for long-term storage (Shi and Burn, 2004). In light of the energy balance equation, obesity represents a form of energy surplus. It is commonly believed to be a result of either increased energy intake and/or decreased energy expenditure. The absolute calorie intake was reduced by $15 \%$ in $\mathrm{Nedd} 4^{+/-}$male mice and by $12 \%$ in $N e d d 4^{+/-}$females compared to their respective wild-type controls. However, when adjusting daily calorie intake for per g body weight, we found no significant difference between gender-matched $\mathrm{Nedd} 4^{+/-}$and wild-type mice. Basal phosphorylation levels of mTOR and AMPK in hypothalamus were similar between genotypes. Direct intracerebroventricular (i.c.v.) injection of leptin into mice evoked similar degree of increase in STAT3 phosphorylation in the two genotypes (see Appendix). These data suggest unaltered energy intake in HFD-fed $\mathrm{Nedd} 4^{+/-}$mice compared to the wild-type controls. These data, however, do not rule out the possibility of altered energy absorption rate by the intestinal tract in $N e d d 4^{+/-}$mice on HFD, which requires taking the energy remnant in urine and feces into consideration.

The body's thermogenesis at rest (basal metabolic rate) comprises a large proportion (60-70\%) of energy expenditure. However, accurate measurement of the basal metabolic rate in mouse present a challenge, as the animals are small and their physical activities are impacted by environmental changes. In addition accurate analysis of the energy expenditure data versus body size is difficult, because it requires advanced equipment (Speakman, 2013). Nevertheless, alteration of basal metabolic rate is normally associated with changes in metabolism in BAT or skeletal muscle, which can be indexed by the expression of thermogenic genes and tissue mass. According to our analysis, the ratios of BAT and skeletal muscle weight to body weight were similar between genotypes with or without HFD feeding. Thermogenesis-related gene expression in BAT and 
expression of UCP1 in BAT, WAT or skeletal muscle were also unchanged by NEDD4 downregulation. Moreover, physical activity as measured in afternoon by open-field test did not show any difference. These data suggest that the energy expenditure may be unaffected in the HFD-fed Nedd $4^{+/-}$mice. Again, these data do not rule out a possible contribution of thermogenesis, as there might be activity change in $\mathrm{Nedd} 4^{+/-}$mice during other untested time spectrum, considering the fact that mice are nocturnal animals.

A third component in energy balance is the efficiency of energy storage. In times of positive energy balance, adipose tissue expands through adipogenesis and lipogenesis to store excess energy in the form of triglycerides, serving as a body defense against the overexposure of excessive lipid; in times of negative energy balance, adipose tissue is mobilized through the lipolysis process to release FFAs, which can be oxidized by other organs as fuel. We hypothesizes that the adipogenesis, lipogenesis, and/or the lipolysis pathways are altered in $N e d d 4^{+/-}$mice on HFD. Indeed, our data imply that the pathways of lipolysis and adipogenesis may contribute to the "lean" phenotype of HFD-fed Nedd $4^{+/-}$mice. By performing in vivo and ex vivo lipolysis assays, we confirmed that at least the lipolytic action was increased in HFD-fed $N e d d 4^{+/-}$mice. In addition, knocking down NEDD4 in 3T3-L1 preadipocytes inhibited its conversion to mature adipocytes; we also observed an associated reduction of PPAR $\gamma$ expression levels in WAT in these mice, which implies that the adipogenesis process may be affected as PPAR $\gamma$ is the central adipogenic transcriptional factor.Besides adipogenesis and lipolysis, the de novo lipogenesis (DNL) is another important contributor to fat accumulation. DNL pathway can be activated by HFD and can take place in both liver and adipose tissue (Strable and Ntambi, 2010). In our studies, $N e d d 4^{+/-}$mice were protected against HFD-induced liver steatosis, raising the possibility of decreased DNL in liver and/or adipose tissue. Indeed, the yeast homologue of NEDD4, Rsp5p, has been demonstrated to regulate unsaturated fatty acid synthesis (Hoppe et al., 2000). In Hoppe et al.'s study, they investigated the physiological outcomes in yeast cells harboring mutant Rsp5, and found that Rsp5 is a vital factor for the survival of yeast cells. To identify target genes for Rsp5, they screened strains carrying yeast library cDNAs to identify strains that could restore the toxic effects of Rsp5 deletion. Finally, the OLE1 gene which encodes stearoyl CoA desaturase (SCD, also called $\Delta 9$ fatty acid desaturase) was confirmed as a target gene of Rsp5 by ubiquitinating and activating the yeast transcription factors SPT23 and MGA2 (homologous to mammalian NF- $\mathrm{kB}$ ). In mammals, SCD controls the synthesis of oleic acid, a form of monounsaturated fatty acids with important physiological functions. Intriguingly, depletion of $S C D-1$ protected mice against obesity and liver steatosis (Cohen et al., 2002; Ntambi et al., 2002), coincidently reminiscent of the $\mathrm{Nedd} 4^{+/-}$mice phenotypes. Thus, it is of particular interest to investigate the effect of NEDD4 on lipogenic pathways. Particularly, it is interesting to see whether the effect of Nedd4 haploinsuffciency on obesity is mediated through SCD, or through its interplay with PPAR $\gamma$ and other lipogenic and adipogenic factors.

Using an obesity model induced by HFD, we demonstrate that NEDD4 is a modulator of lipid accumulation and is a novel regulator of adiposity in mice. Of note, drastic differences in weight gain between the two genotypes were only observed in HFD-fed group. Although we also observed a trend for decreasing body weight with 
aged-obese $\mathrm{Nedd} 4^{+/-}$mice, the data failed to display statistical significance, presumably due to small cohort size and great individual variation.

\subsubsection{Peripheral Insulin Sensitivity in Nedd4-haploinsufficient Mice}

Cao et al. reported reduced insulin signaling in NEDD4 knockout MEFs. Similarly, we also observed decreased insulin sensitivity in $N e d d 4^{+/-}$mice at postnatal stage during ITT. Insulin signaling in several insulin-responsive organs of $\mathrm{Nedd} 4^{+/-}$mice was also impaired. However, we did not observe significantly increased expression of either Grb10, as reported by Cao et al., or PTEN, a previously identified NEDD4 substrate in cancer and neuronal cells (Kwak et al., 2010; Wang et al., 2007), suggesting cell-type-dependent substrate specificity for NEDD4. Despite the insulin tolerance defect, ND-fed $\mathrm{Ne} d d 4^{+/-}$mice were paradoxically glucose tolerant with normal basal blood glucose level. Collectively, the data suggest that ND-fed $N e d d 4^{+/-}$mice possess only mildly decreased insulin sensitivity, which may be compensated by increased insulin secretion through mild $\beta$ cell hypertrophy and/or hyperplasia. Surprisingly, chronically HFD-fed $\mathrm{Ned}_{d} 4^{+-}$mice displayed the same degree of glucose intolerance despite lower circulating insulin levels, compared to HFD-fed controls. This dissociation of insulin resistance from body fat was observed in other murine models - such as the PPAR $\gamma 2$ knockout (Medina-Gomez et al., 2007; Medina-Gomez et al., 2005; Zhang et al., 2004), the Txnip knockout (Chutkow et al., 2010), the Pref-1 overexpressing (Lee et al., 2003), and the adiponectin overexpressing (Kim et al., 2007) — which is similar to the phenotypes of PPAR $\gamma$ agonist treatment (Lehrke and Lazar, 2005). The adiponectin transgenic mice on the $o b / o b$ background exhibited expanded adiposity, which was, surprisingly, associated with improved metabolic outcomes, such as increased insulin sensitivity and glucose tolerance (Kim et al., 2007). While in the case of PPAR $\gamma 2$ knockout and Pref-1 overexpressing mice, adipose mass was profoundly reduced, yet was associated with several metabolic abnormalities including insulin resistance. In contrast to the adipocentric view which considers the excessive fat storage as the main cause of insulin resistance and T2D (Cederberg and Enerback, 2003), these evidence suggest that the fat volume unlikely contributed directly to the obesity-associated insulin resistance. Rather, the expandability of adipose tissue and the ectopic lipotoxicity may be the primary factors linking obesity and insulin resistance (Virtue and Vidal-Puig, 2010).

An unexpected finding from our work is that although $\mathrm{Nedd} 4^{+/-}$mice on ND were resistant to insulin-mediated glucose clearance effect during ITT, GTT by i.p. glucose injection did not reveal difference between the two genotypes. This observation of impaired insulin tolerance yet normal glucose tolerance is coincidently similar to the phenotypes of PPAR $\gamma 2$ knockout mice, suggesting that NEDD4 and PPAR $\gamma 2$ might regulate overlapping molecular pathways in the control of insulin sensitivity(Zhang et al., 2004). Furthermore, in a state of energy surplus evoked by HFD, Nedd $4^{+/-}$mice were, paradoxically, protected against obesity, yet did not become more insulin resistant. In contrast to the deleterious effects on insulin sensitivity by 16 weeks of HFD seen in wildtype mice, insulin sensitivity in $N e d d 4^{+/-}$mice as assessed by ITT did not further deteriorate. These phenotypes are, again, reminiscent of the phenotypes of $P P A R \gamma^{+/-}$mice; 
these mice were protected against obesity an insulin resistance induced by HFD (Kubota et al., 1999). Our Western-blot analysis of PPAR $\gamma$ protein levels in adipose tissue from $\mathrm{Nedd}^{+/-}$and wild-type mice under HFD-fed or aged conditions revealed a positive correlation between NEDD4 and PPAR $\gamma$ levels, though the changes in PPAR $\gamma$ expression levels under aged condition failed to achieve statistical significance. The levels of NEDD4 were better correlated with PPAR $\gamma 2$ than with PPAR $\gamma 1$ under HFD-fed condition, but were correlated similarly with both isoforms under aged condition. These results imply that NEDD4 may be an upstream regulator of PPAR $\gamma$, and that the metabolic effects of NEDD4 may be mediated through PPAR $\gamma$, especially PPAR $\gamma 2$.

In terms of the discrepancy that the "insulin resistant predisposed"Nedd $4^{+/-}$mice did not become more insulin resistant, as is also the case with $P P A R \gamma^{+/-}$mice, one possible explanation might be that these mice already had reduced insulin sensitivity on a ND; the molecular mechanisms through which their partial deficiency leads to insulin resistance on the ND are very similar to the mechanisms underlying insulin resistance induced by a HFD, and therefore insulin resistance did not become further impaired (Medina-Gomez et al., 2005). Another possibility is that reduced adiposity in HFD-fed these mice could result in a modest improvement in insulin sensitivity, which might compensate the additive effect of haploinsufficiency on HFD-induced insulin resistance. Mechanisms underlying these phenotypes thus far only partially understood. Deeper understanding of the regulation, signaling, and tissue-specific roles of NEDD4 could provide novel mechanistic insights and new therapeutic targets for its actions related to obesity and insulin resistance.

\subsubsection{Regulation of Lipolysis by NEDD4}

A study conducted by Langin et al. showed that obesity is associated with resistance to catecholamine-induced lipolysis (Langin et al., 2005), suggesting that enhancing lipolytic rate is a potential strategy to remove fat and treat obesity. Although there is a widespread acceptance of the notion that an increase in lipolysis would result in facilitated release of FFAs into bloodstream, thereby mediating several adverse metabolic effects, including insulin resistance (Nielsen et al., 2004). This idea is now being challenged by recent studies. Clinically, even severe insulin resistance, can coexist with obesity with no alteration in FFA levels. On the other hand, increased levels of FFAs are not necessarily associated with insulin resistance (Karpe et al., 2011), suggesting that the increased levels of plasma FFA associated with obesity is a consequence rather than a cause (Byrne et al., 1999). Furthermore, enhanced lipolysis does not change serum FFAs levels, as demonstrated in several murine models(Ahmadian et al., 2009; Ahmadian et al., 2010; Jaworski et al., 2009; Saha et al., 2004; Tansey et al., 2001). Therefore, promoting fat loss by enhancing lipolysis may still be an intriguing strategy for the treatment of obesity.

In human, catecholamines mainly epinephrine and norepinephrine are the hormones with pronounced lipolytic action via the seven transmembrane protein $\beta$-AR. Three distinct $\beta$-AR subtypes have been identified including $-\beta_{1}-\mathrm{AR}, \beta_{2}$-AR and $\beta_{3}-\mathrm{AR}$. 
The $\beta$-AR-mediated lipolytic process in adipose has been extensively studied in human and rodent adipocytes. However, the human and rodent adipocytes have differential responsiveness to $\beta_{3}$-AR agonists to activate the lipolysis pathway. Human adipocytes respond much less efficiently to the $\beta_{3}$-AR agonist. Functional assays by applying $\beta$ agonists indicate that catecholamines primarily activate $\beta_{1}-A R$ and $\beta_{2}-A R$, rather than the $\beta_{3}-\mathrm{AR}$ in both white and brown adipocytes from rats (Bahouth and Malbon, 1988; Galitzky et al., 1995). Although the $\beta_{3}$-AR is the only $\beta$-AR specifically expressed in fat, RT-PCR results from a previous report suggest that $\beta_{1}$-AR and $\beta_{2}$-AR are the major subtypes in the subcutaneous adipose tissue from rat (McNeel and Mersmann, 1999), consistent with the functional assays. Our studies show that isoproterenol-stimulated lipolytic response in $\mathrm{Nedd} 4^{+/-}$mice on HFD is remarkably higher than that from their wild-type littermates on HFD. Since the $\beta_{3}$-AR subtype does not significantly contribute to isoprenaline-induced lipolysis (Tavernier et al., 1996). We thereby speculate that the effect is primarily through $\beta_{1}$ - or $\beta_{2}-\mathrm{AR}$.

The $\beta$-AR belongs to a $\mathrm{G}$ protein coupled receptor (GPCR) family. Sustained activation of the $\beta$-ARs leads to receptor desensitization and/or degradation. While the $\beta_{1}$-AR is believed to be resistant to agonist-induced ubiquitination and degradation (Liang and Fishman, 2004), the E3 ligase-mediated ubiquitination of $\beta_{2}$-AR plays an important role in regulating the abundance of the receptor. NEDD4 is known to be recruited to the agonist-activated $\beta_{2}$-AR via two adaptor proteins: $\beta$-arrestin 2 and arrestin-domain-containing proteins (ARRDCs), especially ARRDC2-4 (Han et al., 2013b; Nabhan et al., 2010; Shenoy et al., 2008). Shenoy et al. demonstrated that MDM2 mediates ubiquitination of $\beta_{2}-\mathrm{AR}$ and their adaptor protein $\beta$-arrestin 2 which regulates the initial step of receptor endocytosis (Shenoy et al., 2001), and NEDD4 mediates subsequent receptor ubiquitination which targets receptors for lysosomal degradation (Shenoy et al., 2008). Although discrepant results from Nabhan et al. suggest that ARRDC3, rather than $\beta$-arrestin 2, serves as the adaptor protein (Nabhan et al., 2010), both studies are not mutually exclusive in that they unequivocally delineate the role of NEDD4 in downregulating $\beta_{2}$-AR. Later, Han et al. demonstrated that the $\beta$-arrestin 2 is the first adaptor protein between NEDD4 and $\beta_{2}$-AR to facilitate clathrin-dependent internalization, while the ARRDCs serve as the secondary adaptors lead the NEDD4 and $\beta_{2}$-AR complex to the early endosome (Han et al., 2013b). Interestingly, ARRDC3deficient mice also display partial resistance to obesity via modulating $\beta$-adrenergic signaling (Patwari et al., 2011). In spite of the fact that NEDD4 has a substantial number of substrates, very few in vivo studies have been reported to validate its pathophysiological relevance. It should be stressed that our work, for the first time, demonstrates a physiological role of NEDD4-mediated $\beta_{2}$-AR signaling in the context of lipolysis.

Even though agonist-stimulated lipolysis was higher in the HFD-fed Nedd4 ${ }^{+/-}$ mice, circulating lipid concentrations did not increase. This is consistent with phenotypes reported in adipose ATGL transgenic mice (Ahmadian et al., 2009), adipocyte phospholipase A(2) (AdPLA) knockout mice (Jaworski et al., 2009), perilipin knockout mice (Saha et al., 2004; Tansey et al., 2001). In these mice, the serum FFAs levels were not altered, even though they all exhibited enhanced lipolysis. Reduced adipose mass 
could have resulted in lower basal FFA release, which might have compensated for the elevated lipolysis in the HFD-fed Nedd $4^{+/}$mice. Alternatively, increased $\beta$-oxidation at ectopic sites could have maintained the circulating lipid homeostasis, although in the current study, we do not have evidence to support this hypothesis. In agreement with our speculation, the murine models mentioned above all exhibited enhanced $\beta$-oxidation at least in adipose tissue (Ahmadian et al., 2009; Jaworski et al., 2009; Saha et al., 2004). One discrepancy from these studies is that, we only observed enhanced lipolysis in HFDfed $\mathrm{Nedd}^{+/-}$mice under the stimulation $\beta$-agonists, while the basal lipolysis appears to be unaffected, which implies that the altered lipolysis in these mice is ligand dependent. This may also explain the discrepancy that the lipolytic response was only affected in the HFD-fed or aged animals, as chronic dietary fat or aging could act as a stressor that stimulates sympathetic nerve activity, releasing catecholamines which facilitates energy expenditure primarily through thermogenesis and $\beta$-AR mediated lipolysis (Lowell and Bachman, 2003).

\subsubsection{Regulation of Adipogenesis by NEDD4}

Chronic adipose tissue remodeling represents a fundamental and highly coordinated process involving the participation of different cell types, including endothelial precursors, immune cells, and preadipocytes (Sun et al., 2011). In mice at an earlier stage of obesity (within the 16-week design of HFD feeding), adipose tissue expands primarily through hypertrophy. At later stages (after 16 weeks of HFD feeding), a high degree of adipocyte apoptosis (approximately $80 \%$ ) becomes evident, so that adipose expansion relies on hyperplasia (Strissel et al., 2007).

Adipogenesis contributes to the expansion of adipose tissue; therefore, pharmacological intervention to inhibit adipogenesis appears to be a logically promising approach to restrain obesity. However, factors that impede adipocyte growth and expansion also lead to metabolic defects (Stephens, 2012). For example, Pref-1 is a negative regulator of adipogenesis secreted by preadipocytes; Pref- 1 transgenic mice are insulin resistant despite its resistance to HFDIO (Villena et al., 2008). Another example is PPAR $\gamma 2$ knockout mice, which also display insulin resistance (Medina-Gomez et al., 2007); PPAR $\gamma 2$ is generally believed to be more effective in pro-adipogenesis than PPAR $\gamma 1$. Hence, factors that limit adipose expansion without altering insulin sensitivity should receive more attention since they represent a promising strategy for weight loss.

Although our work has revealed that NEDD4 and PPAR $\gamma$ expressions are positively correlated and that these two proteins can be co-immunoprecipitated, future studies must consider the following key questions. Are the NEDD4 and PPAR $\gamma$ physically interacted, or through an adaptor? Does the NEDD4-PPAR $\gamma$ interaction account for NEDD4's regulation of PPAR $\gamma$ expression? Where does the interaction occur? What is the physiologic consequence of attenuating the NEDD4-PPAR $\gamma$ interaction during adipocyte differentiation? During our investigation, a study from Han et al. reported the physical interaction between PPAR $\gamma$ and NEDD4 through PPxY motif in an overexpression in vitro system. They claimed that NEDD4 is the E3 ligase for PPAR $\gamma$ in 
response to the 17 $\beta$-estradiol treatment in HeLa cells (Han et al., 2013a). However, in our study, we did not see a significant reduction in PPAR $\gamma$ levels by NEDD4 overexpression, suggesting that PPAR $\gamma$ is subjected to the regulation of different E3 ligases under different conditions. In the current study, we observed in vitro that knockdown of NEDD4 in 3T3-L1 preadipocytes reduced PPAR $\gamma$ protein expression and inhibited adipogenesis. We propose that NEDD4 interacts and stabilizes PPAR $\gamma$ in preadipocytes when PPAR $\gamma$ level is low, prohibiting its degradation by other E3 ligases, whereas NEDD4 disassociates from PPAR $\gamma$ and releases it for nuclear translocation and transactivation (Hauser et al., 2000) during the adipocyte differentiation process (Figure 6.1). Due to the high lipid content of adipose cells, it is technically difficult to study PPAR $\gamma$ localization and trafficking in vivo. Using the 3T3-L1 preadipocytes, Thuillier et $a l$. showed that PPAR $\gamma$ distributes evenly in the cytosol and nucleus of the preadipocyte; whereas during differentiation, PPAR $\gamma$ increases in the cytosol while remaining constant within the nucleus (Thuillier et al., 1998). Localization of NEDD4 in adipocytes has not been reported, but NEDD4 localizes to both cytoplasm and nucleus in most cell types. The NEDD4/PPAR $\gamma$ complex probably derives from this overlapping protein pool. Since NEDD4's effects on lipolysis and adipogenesis can be reproduced in vitro (at least in 3T3-L1 cells), these observations are unlikely to be secondary consequences from other organs, such as muscle and liver. Tissue-specific signaling and function of NEDD4 are worthy for further investigation using conditional knockout mice.

\subsubsection{E3 Ligases in Metabolism}

E3 ligase is the final step for the conjugation of ubiquitin to substrates managing the selection of targets, and therefore has been substantially studied in diseases with abnormal protein accumulation, such as neurodegeneration and cancer (Ardley and Robinson, 2004; Sun, 2003). There has been very limited investigation of this class of enzymes for the metabolic functions. Rsp5p, the most well-studied yeast E3 ligase, participates in the process of unsaturated fatty acid synthesis (Hoppe et al., 2000). Its mammalian homologues - NEDD4 and ITCH (also called AIP4) - are two structurally related E3 ligases belonging to the nine-member HECT NEDD4 family. NEDD4 and ITCH both recognize the proline-rich consensus sequence motifs (e.g., PPxY and PPLP) and thereby ubiquitinate several overlapping targets in vitro (Bray, 2006), including the RING-type E3ligase Cbl-b (Magnifico et al., 2003). Comparative study of immunity using genetically modified mice indicates that NEDD4 and ITCH regulate distinct pathways in vivo (Yang et al., 2008). Recently, depletion of ITCH in mice has been found to reverse HFD-induced obesity and its related metabolic disorders, as well as an aberrant M2 macrophage polarization (Marino et al., 2014). Our work shows that NEDD4 partial depletion also attenuates HFDIO progression and pro-inflammatory response, likely through decreased M1 macrophage recruitment. These findings suggest that distinct metabolic and inflammatory pathways regulated by NEDD4 and ITCH may converge to produce an anti-obese action. The NEDD4/ITCH and Cbl-b were frequently found to be mutually impeded in previous studies (Guo et al., 2012; Thien and Langdon, 2005). Coincidentally, Cbl-b depletion caused aggravated HFD-induced insulin resistance and macrophage recruitment (Abe et al., 2013; Abe et al., 2014), consistent with its inverse 
Figure 6.1 Schematic Diagram of NEDD4 Regulation of Lipolysis and Adipogenesis. Under pathological conditions such as aging or HFD-induced obesity, plasma levels of catecholamine and FFA increase. The increased presence of catecholamine promotes ligand-induced endocytosis and degradation of $\beta_{2}$-AR mediated by NEDD4, resulting in fewer receptors on the surface of adipocyte and reduced lipolysis. On the other side, elevated FFA uptake into adipocyte provides more ligands for PPAR $\gamma$. NEDD4 interacts and stabilizes PPAR $\gamma$, prohibiting its degradation by other E3 ligases and facilitates adipogenesis mediated through PPAR $\gamma$ and RXR as heterodimer. Although detailed regulation of this process by NEDD4 await further investigation. 


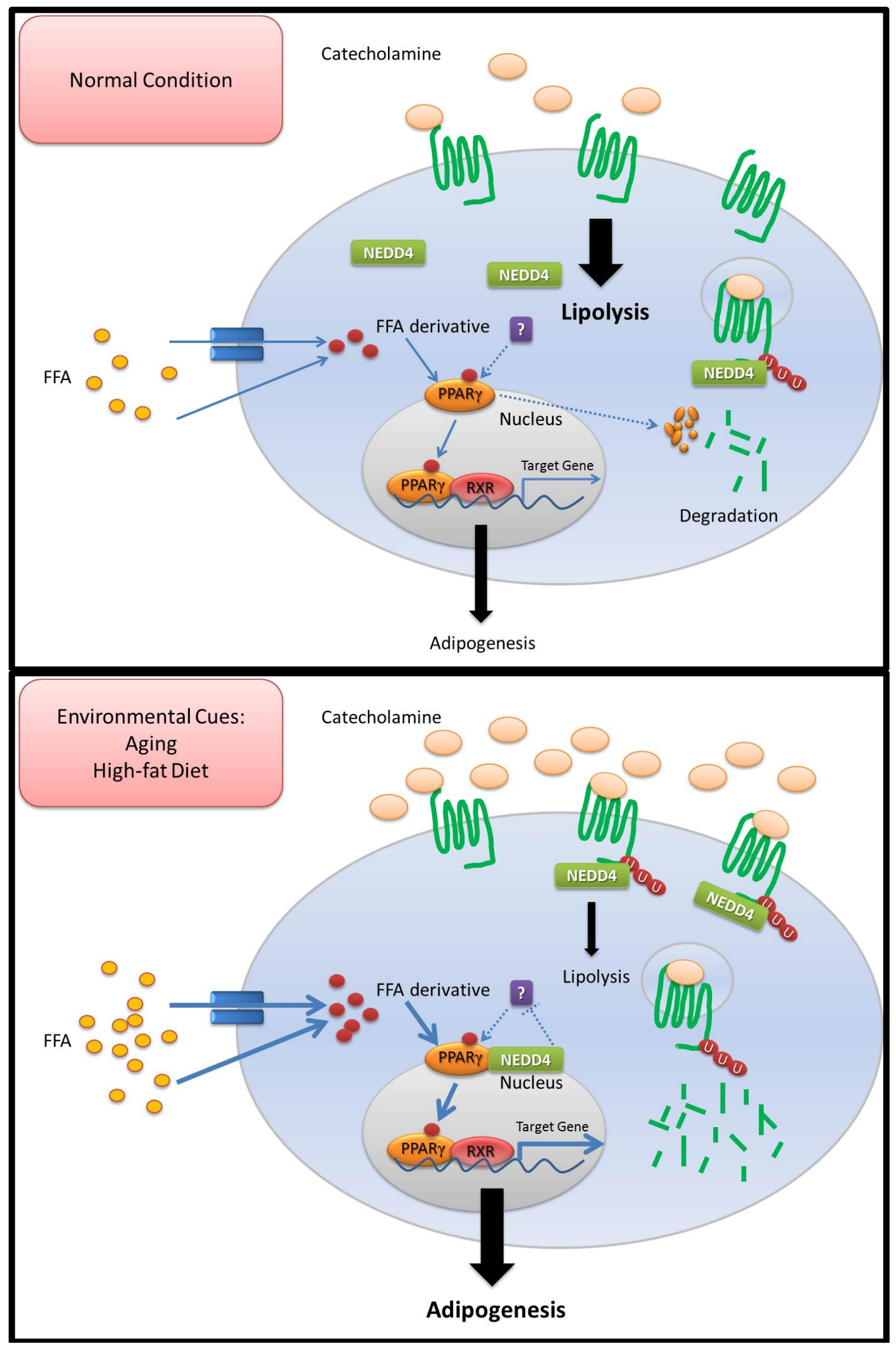


relationship to NEDD4/ITCH. The crosstalk of molecular pathways among these three E3 ligases in obesity is still waiting to be further investigated.

\subsection{Physiological Role of NEDD4 in CNS}

\subsubsection{Nedd4 Haploinsufficiency Leads to Altered Cognitive and Anxiety-like Behaviors}

In our studies, adult $\mathrm{Nedd} 4^{+/-}$mice showed significant impairment in behavioral by the water maze test. Overall tissue pathological alteration was not observed. Previously, $\mathrm{Ned} 4^{+/-}$mice were reported to have deficits in the development of cardiac and neuromuscular junction (Fouladkou et al., 2010; Liu et al., 2009). This raises the question that if the impaired water maze performance in $N e d d 4^{+/}$mice might be unrelated to their cognitive functions. In our current study, physiological parameters, such as organ weights, in Nedd $4^{+/}$mice were similar to those of wild-type counterparts. During the visible platform training, $N e d d 4^{+/-}$mice performed well but only displayed impairment in the hidden section. This pattern of behavior indicates that the $\mathrm{Nedd} 4^{+/-}$ mice does not have visual problems and suggest a cognitive deficit. These findings implicate NEDD4 in certain unidentified processes underlying neuritic plasticity in the mammalian brain, involving the hippocampal formation. This is the first physiological evidence of NEDD4 in potential regulation of learning and memory.

As $\mathrm{Ne}_{\mathrm{d}} \mathrm{4}^{+/-}$mice performed differently in cross maze, fear conditioning and water maze, we are unable to conclude so far whether this discrepancy is a result of the different settings in experimental paradigms or indicates a differential regulation of shortterm and long-term memory by endogenous NEDD4. Future studies may need to include more behavioral tests to assess their learning and memory ability in different environments. In the current study, we also demonstrated that NEDD4 is involved in the development of anxiety-like behaviors as judged by open-field and light/dark box tests. The mechanisms underlying these neurobehavioral changes remain unknown. It should be pointed out that water maze test is well known to cause severe anxiety on mice; mice, compared to rats, have intrinsic fear of water. Therefore, in this case, water maze may not be a suitable test for long-term memory. Instead, a dryland version called Barnes maze test should be used to clarify whether $N e d d 4^{+/-}$mice indeed have impaired longterm memory.

\subsubsection{Central Insulin Signaling in Nedd4-haploinsufficient Mice}

Insulin and IR signaling are known to play critical roles in the process of learning and memory (Zhao and Alkon, 2001; Zhao et al., 2004), however, examination of insulin sensitivity locally in the hippocampus, especially in the adults, presents a challenge. Because brain is extremely sensitive to hypoxia; death of neurons is prominent even immediately following the isolation of the brain. To test the central insulin signaling in 
Nedd $4^{+/}$mice, we tried to administer insulin through i.p., intravenous (i.v.), and i.c.v. routes. None of these methods provided reasonably accurate measurements. Due to the existence of blood brain barrier, large molecules like insulin cannot be efficiently delivered to the brain through i.p. or i.v. injection, since its transport requires a saturable transporter (Banks et al., 1997). The concentration of insulin in the brain is, therefore, largely dependent upon the transport efficiency. Insulin signaling is an acute effect which can trigger phosphorylation events within 5 min of exposure to insulin. Furthermore, hippocampus is less sensitive to insulin than regions like hypothalamus. Therefore, i.p. or i.v. injections of insulin only induced a very mild degree of phosphorylation in IR in hippocampus; also, a large variation between individual mouse was shown which is likely due to the different transport efficiency of insulin in each mouse.

I.c.v. injection allows insulin to merge locally to the cerebrospinal fluid within cerebral lateral ventricle and circulate to hippocampus. We did see a robust induction of IR phosphorylation with i.c.v. injection. However, the data were difficult to interpret as we saw increased IR phosphorylation levels in Nedd $4^{+-}$mice, which are opposite to the peripheral insulin signaling data. We suspect that the ketamine/xylazine which we used to anesthetize the mice before surgery may have compromised our data, as ketamine and xylazine have been implicated in the regulation of insulin sensitivity (Heim et al., 2002; Xiao et al., 2013). In the present study, we stimulated the cortical tissue isolated from P0 mice with insulin. Use of cortical tissue from neonatal mice has the advantage of being relatively resistant to hypoxia therefore the isolated tissue not need for oxygen supplement within the first 2 hours to maintain the viability of neurons. Shortcomings are that the response only represents neonatal mice but not adult mice and that it is difficult to study region-specific effects.

\subsection{Future Studies}

As discussed previously, to better solve mechanistic puzzle of tissue-specific regulation by NEDD4 in adipocyte, a conditional knockout of NEDD4 in adipose tissue can be considered. This will help circumvent the potential problems from CNS controls, as the WAT is innervated by the sympathetic nervous system (SNS). Bioinformatics tools including next generation RNA sequencing and proteomics may be employed to further delineate cell-type specific substrates of NEDD4 in adipocytes.

To improve data quality, the metabolic cages could be considered for measurement of basal metabolic rate and energy intake where the energy remnant in the urine and feces can be carefully subtracted; a Dual-energy X-ray absorptiometry technique could be utilized to measure lean/fat mass ratios and the hyperinsulinemic-euglycemic clamp technique may be employed to assess insulin sensitivity. In addition, lentivirus has been reported to have better infection rate than AAV for 3T3-L1 cells and therefore, may improve the knockdown efficiency of NEDD4 and produce more significant results. Alternatively, inducing adipogenesis using NEDD4 knockout MEF cells may be considered. However, it should be noted that the differentiation efficiency in primary MEFs varies from $10-70 \%$ and immortalized MEF cells normally require the 
overexpression of a pro-adipogenic transcription factor such as PPAR $\gamma$ or C/EBP $\alpha$ (Rosen and MacDougald, 2006). Future experiments could be designed to analyze the interaction sites between NEDD4 and PPAR $\gamma$ and clarify the physiological relevance of their interaction. It would be of particular interest to see the metabolic outcome in mice or adipocytes with the expression of PPAR $\gamma$ lacking the binding sequences for NEDD4.

The $N e d d 4^{+/-}$mice are partially protected against HFD-induced obesity, implying that NEDD4 may mediate the development of obesity to some extent. Surprisingly, the several metabolically active tissues (hypothalamus, skeletal muscle and WAT) from HFD-fed mice is not associated with aberrant NEDD4 protein expression. We speculate that NEDD4 may be altered in a specific cell type (e.g. the preadipocytes). If so, measuring the expression of NEDD4 in tissues with mixed cell types may yield false information. Another plausible explanation is that NEDD4's activity is changed rather than its expression levels. NEDD4 protein contains multiple catalytic cysteine residues which are vulnerable to oxidative stress normally associated with obesity.

To confirm the behavioral phenotypes of $N e d d 4^{+/-}$mice, additional behavioral tests such as Barnes maze, Y maze, and novel object recognition tests for spatial learning and memory may be considered. Moreover, elevated plus maze and novelty-suppressed feeding test may be performed to validate the anxiety-like behavior in $\mathrm{Nedd} 4^{+/-}$mice. Single-cell RNA sequencing and proteomics which avoid glial cell contamination may be considered for further delineation of NEDD4 substrates in neurons. Future investigation of insulin signaling in adult brain may consider using acute hippocampal slice preparation following tissue slice electrophysiology experiments. This technique circumvents contamination from anesthetics and reduces animal use, as both dose- and time-response studies can be performed in slices from a single mouse.

\subsection{Using Heterozygous Mouse as Models-Advantages vs. Disadvantages}

Over the past two decades, genetically modified mice have provided us with tremendous advantages in studying physiological functions of molecules in vivo (in mammals), more specifically by manipulating their gene expression. Currently, conditional knockout and transgenic technologies enable researchers to specifically delete or overexpress certain genes at particular stages of life. In addition, it allows researchers to do so in a tissue-specific or cell-type specific manner. These available technologies greatly advance our understanding of the patho-physiological roles of a certain molecule or molecular pathway in areas and at times of interest. However, limitations of this technique remain with respect to controlling the expression level of a transgene, which are so called dosage-dependent effects. Therefore, caution should be taken when interpreting mouse phenotypes. For example, when knocking out a certain gene resulting in morbidity or mortality, it is not implicit that its overexpression will necessarily lead to beneficial effects. Often, to study a gene's expected function by complete knockout or overexpression may be compromised by embryonic lethality as in the case of $\mathrm{Nedd} 4^{-/-}$ mice. The embryonic lethality indicates the crucial role of NEDD4 in IGF-1 signaling of the early developmental stages, while overexpression of NEDD4, as in the case of 
neurodegeneration, leads to impaired IGF-1 signaling due to its role as the E3 ligase of IGF-1R. Ultimately knockout and overexpression approaches are often used to study physiological and pathological functions of a molecule, respectively. Expressing a transgene at "right dosages" is of particular importance especially in disease-related research.

Because it is uncommon for human molecules to be completely knocked out or overexpressed, a more practical approach for study entails heterozygosity. In our studies, we used a Nedd4 heterozygous mouse model to characterize the role of the Nedd4 gene in metabolism and CNS functions. Though partial depletion of Nedd4 was sufficient to produce a certain degree of metabolic phenotypes, the phenotypes observed were generally mild. For example, $\mathrm{Ned} d 4^{+/-}$mice exhibited moderate insulin resistance, because their glucose clearance responded less efficiently to insulin during ITT. Additionally, they had slightly higher serum insulin levels when compared to their wildtype littermates. Nedd $4^{+/-}$mice were only partially resistant to HFD-induced obesity (30\% reduction in weight gain) without improvement in obesity-associated insulin resistance, hyperglycemia, and hyperlipidemia. The extent of PPAR $\gamma$ downregulation was at most $50 \%$. Although using a diet with a lower percentage of calories from fat may help enlarge the difference, whether these phenotypes or results can become more dramatic or significant when Nedd4 is completely depleted is unknown. We believe that WAT is the most selectively affected tissue under obesogenic conditions as demonstrated by our ex vivo and in vitro data. Using heterozygous mice for an initial characterization offers insight to the potential pathways affected. Further studies using tissue-specific conditional knockout mice will be necessary to fully validate our hypotheses, which may further advance our knowledge of the biological functions of NEDD4 in metabolism.

One should bear in mind the potential artifacts of the observed phenotypes displayed through a genetically manipulated mouse model. The specificity of knockout or knockdown effects in gene-trap studies has been questioned, because these mice are hybrid in origin with both B6 and 129 type alleles. The 129-type alleles could also contributes to the changes in phenotypes (Muller, 1999). The mutant mice in our present study were heterozygous for not only the Nedd4 locus, but also the flanking region (B6 $\times$ 129). It has been reported that the C57BL/6J (B6) mouse strain was more prone to obesity, while the 129Sv strain was both obesity- and diabetes-resistant. Accordingly, their intercross $(\mathrm{B} 6 \times 129) \mathrm{F} 2$ mice were more obese-prone than 129 mice (Almind and Kahn, 2004). In terms of neurobehavior, F1 hybrids of B6 and 129 strains perform better than both inbred B6 and 129 strains in learning and memory tasks (Crawley et al., 1997). Therefore, the resistance to diet-induced obesity and the behavioral deficits observed in the $\mathrm{Nedd} 4^{+/}$mice of B $6 \times 129$ hybrid origin are not likely explained by the contribution of 129-type alleles.

\subsection{E3 Ligase as Drug Target}

Dysregulation of UPS is associated with a number of hard-to-treat diseases, including cancer, neurodegeneration, viral infection, muscle atrophy, and diabetes and 
inflammation. The reversible proteasome inhibitor bortezomib (Velcade ${ }^{\circledR}$ ) was the first FDA approved drug for the treatment of both multiple myeloma and mantle cell lymphoma (Adams and Kauffman, 2004). The following year, the Nobel Prize in Chemistry was awarded to jointly to Aaron Ciechanover, Avram Hershko, and Irwin Rose for their discovery of UPS pathway (The official website of the Nobel Prize). In the past decade, UPS-pathway-targeting compounds against more precise targets have emerged as novel candidates for drug discovery. Considering the fact that humans possess two E1 enzymes, $30 \mathrm{E} 2$ enzymes and several hundreds of E3 ligases, compounds that target E1, or E2 enzymes are hopeless due to lack of specificity, and thereby could result in undesirable side-effects. E3 ligases control the specificity of substrate ubiquitination, making this class of enzymes the most appealing targets for drug development. However, the E3 ligase targeting drug discovery process is still in its infancy due to the complexity of UPS regulations (Wilkie et al. 2012). Our studies provide experimental evidence for the involvement of NEDD4 E3 ligase in several biological processes (lipolysis, adipogenesis, insulin signaling, learning/memory, and anxiety), which may facilitate our understanding of complex E3-ligases-mediated UPS regulation in the context of physiological and pathological settings. Full investigation of each pathway in greater details at molecular levels will be instrumental to future validation of NEDD4 as a suitable drug target. 


\section{LIST OF REFERENCES}

(2008). State-specific prevalence of obesity among adults--United States, 2007. MMWR Morb Mortal Wkly Rep 57, 765-768.

Abe, T., Hirasaka, K., Kagawa, S., Kohno, S., Ochi, A., Utsunomiya, K., Sakai, A.,Ohno, A., Teshima-Kondo, S., Okumura, Y., et al. (2013). Cbl-b is a critical regulator of macrophage activation associated with obesity-induced insulin resistance in mice. Diabetes 62, 1957-1969.

Abe, T., Hirasaka, K., Kohno, S., Ochi, A., Yamagishi, N., Ohno, A.,Teshima-Kondo, S., and Nikawa, T. (2014). Ubiquitin ligase Cbl-b and obesity-induced insulin resistance. Endocr J 61, 529-538.

Ahmadian, M., Duncan, R.E., Varady, K.A., Frasson, D., Hellerstein, M.K., Birkenfeld, A.L., Samuel, V.T., Shulman, G.I., Wang, Y., Kang, C., and Sul, H.S. (2009). Adipose overexpression of desnutrin promotes fatty acid use and attenuates diet-induced obesity. Diabetes $58,855-866$.

Ahmadian, M., Wang, Y., and Sul, H.S. (2010). Lipolysis in adipocytes. Int J Biochem Cell Biol 42, 555-559.

Almind, K., and Kahn, C.R. (2004). Genetic determinants of energy expenditure and insulin resistance in diet-induced obesity in mice. Diabetes 53, 3274-3285.

Ardley, H.C., and Robinson, P.A. (2004). The role of ubiquitin-protein ligases in neurodegenerative disease. Neurodegener Dis 1,71-87.

Arner, P. (1995). Differences in lipolysis between human subcutaneous and omental adipose tissues. Ann Med 27, 435-438.

Arner, P., Hellstrom, L., Wahrenberg, H., and Bronnegard, M. (1990). Beta-adrenoceptor expression in human fat cells from different regions. J Clin Invest 86, 1595-1600.

Bahouth, S.W., and Malbon, C.C. (1988). Subclassification of beta-adrenergic receptors of rat fat cells: a re-evaluation. Mol Pharmacol 34,318-326.

Baker, J., Liu, J.P., Robertson, E.J., and Efstratiadis, A. (1993). Role of insulin-like growth factors in embryonic and postnatal growth. Cell 75, 73-82.

Banks, W.A., Jaspan, J.B., and Kastin, A.J. (1997). Selective, physiological transport of insulin across the blood-brain barrier: novel demonstration by species-specific radioimmunoassays. Peptides 18, 1257-1262. 
Benedict, C., Hallschmid, M., Hatke, A., Schultes, B., Fehm, H.L., Born, J., and Kern,W. (2004). Intranasal insulin improves memory in humans. Psychoneuroendocrinology 29, 1326-1334.

Bluher, M., Kahn, B.B., and Kahn, C.R. (2003). Extended longevity in mice lacking the insulin receptor in adipose tissue. Science 299, 572-574.

Bluher, M., Michael, M.D., Peroni, O.D., Ueki, K., Carter, N., Kahn, B.B., and Kahn, C.R. (2002). Adipose tissue selective insulin receptor knockout protects against obesity and obesity-related glucose intolerance. Developmental cell 3, 25-38.

Blundell, J.E., Caudwell, P., Gibbons, C., Hopkins, M., Naslund, E., King, N., and Finlayson, G. (2012). Role of resting metabolic rate and energy expenditure in hunger and appetite control: a new formulation. Dis Model Mech 5, 608-613.

Bray, S.J. (2006). Notch signalling: a simple pathway becomes complex. Nat Rev Mol Cell Biol 7, 678-689.

Buettner, R., Scholmerich, J., and Bollheimer, L.C. (2007). High-fat diets: modeling the metabolic disorders of human obesity in rodents. Obesity (Silver Spring) 15, 798-808.

Byrne, C.D., Maison, P., Halsall, D., Martensz, N., Hales, C.N., and Wareham, N.J. (1999). Cross-sectional but not longitudinal associations between non-esterified fatty acid levels and glucose intolerance and other features of the metabolic syndrome. Diabet Med 16, 1007-1015.

Cai, D., Dhe-Paganon, S., Melendez, P.A., Lee, J., and Shoelson, S.E. (2003). Two new substrates in insulin signaling, IRS5/DOK4 and IRS6/DOK5. J Biol Chem 278, 2532325330 .

Cannon, B., and Nedergaard, J. (2004). Brown adipose tissue: function and physiological significance. Physiol Rev 84, 277-359.

Cao, T.T., Deacon, H.W., Reczek, D., Bretscher, A., and von Zastrow, M. (1999). A kinase-regulated PDZ-domain interaction controls endocytic sorting of the beta2adrenergic receptor. Nature 401, 286-290.

Cao, X.R., Lill, N.L., Boase, N., Shi, P.P., Croucher, D.R., Shan, H., Qu, J., Sweezer, E.M., Place, T., Kirby, P.A., et al. (2008). Nedd4 controls animal growth by regulating IGF-1 signaling. Sci Signal 1, ra5.

Caro, J.F., Sinha, M.K., Kolaczynski, J.W., Zhang, P.L., and Considine, R.V. (1996). Leptin: the tale of an obesity gene. Diabetes 45, 1455-1462.

Cederberg, A., and Enerback, S. (2003). Insulin resistance and type 2 diabetes--an adipocentric view. Curr Mol Med 3, 107-125. 
Chen, Z.J., and Sun, L.J. (2009). Nonproteolytic functions of ubiquitin in cell signaling. Mol Cell 33, 275-286.

Chiang, S.H., Baumann, C.A., Kanzaki, M., Thurmond, D.C., Watson, R.T., Neudauer, C.L., Macara, I.G., Pessin, J.E., and Saltiel, A.R. (2001). Insulin-stimulated GLUT4 translocation requires the CAP-dependent activation of TC10. Nature 410, 944-948.

Chutkow, W.A., Birkenfeld, A.L., Brown, J.D., Lee, H.Y., Frederick, D.W., Yoshioka, J., Patwari, P., Kursawe, R., Cushman, S.W., Plutzky, J., et al. (2010). Deletion of the alphaarrestin protein Txnip in mice promotes adiposity and adipogenesis while preserving insulin sensitivity. Diabetes 59, 1424-1434.

Ciechanover, A. (2005). Proteolysis: from the lysosome to ubiquitin and the proteasome. Nat Rev Mol Cell Biol 6, 79-87.

Clausen, J.O., Borch-Johnsen, K., Ibsen, H., Bergman, R.N., Hougaard, P., Winther, K., and Pedersen, O. (1996). Insulin sensitivity index, acute insulin response, and glucose effectiveness in a population-based sample of 380 young healthy Caucasians. Analysis of the impact of gender, body fat, physical fitness, and life-style factors. J Clin Invest 98 , 1195-1209.

Clement, K., Vaisse, C., Lahlou, N., Cabrol, S., Pelloux, V., Cassuto, D., Gourmelen, M., Dina, C., Chambaz, J., Lacorte, J.M., et al. (1998). A mutation in the human leptin receptor gene causes obesity and pituitary dysfunction. Nature 392, 398-401.

Cohen, P., Miyazaki, M., Socci, N.D., Hagge-Greenberg, A., Liedtke, W., Soukas, A.A., Sharma, R., Hudgins, L.C., Ntambi, J.M., and Friedman, J.M. (2002). Role for stearoylCoA desaturase-1 in leptin-mediated weight loss. Science 297, 240-243.

Craft, S., Baker, L.D., Montine, T.J., Minoshima, S., Watson, G.S., Claxton, A., Arbuckle, M., Callaghan, M., Tsai, E., Plymate, S.R., et al. (2012). Intranasal insulin therapy for Alzheimer disease and amnestic mild cognitive impairment: a pilot clinical trial. Arch Neurol 69, 29-38.

Curran, M.P., and Scott, L.J. (2004). Orlistat: a review of its use in the management of patients with obesity. Drugs 64, 2845-2864.

de Ferranti, S., and Mozaffarian, D. (2008). The perfect storm: obesity, adipocyte dysfunction, and metabolic consequences. Clin Chem 54, 945-955.

Degerman, E., Belfrage, P., and Manganiello, V.C. (1997). Structure, localization, and regulation of cGMP-inhibited phosphodiesterase (PDE3). J Biol Chem 272, 6823-6826.

Dhamoon, M.S., Noble, J.M., and Craft, S. (2009). Intranasal insulin improves cognition and modulates beta-amyloid in early AD. Neurology 72, 292-293; author reply 293-294. 
Dou, J.T., Chen, M., Dufour, F., Alkon, D.L., and Zhao, W.Q. (2005). Insulin receptor signaling in long-term memory consolidation following spatial learning. Learn Mem 12, 646-655.

Drinjakovic, J., Jung, H., Campbell, D.S., Strochlic, L., Dwivedy, A., and Holt, C.E. (2010). E3 ligase Nedd4 promotes axon branching by downregulating PTEN. Neuron 65, 341-357.

Duncan, R.E., Ahmadian, M., Jaworski, K., Sarkadi-Nagy, E., and Sul, H.S. (2007). Regulation of lipolysis in adipocytes. Annu Rev Nutr 27, 79-101.

Elchebly, M., Payette, P., Michaliszyn, E., Cromlish, W., Collins, S., Loy, A.L., Normandin, D., Cheng, A., Himms-Hagen, J., Chan, C.C., et al. (1999). Increased insulin sensitivity and obesity resistance in mice lacking the protein tyrosine phosphatase-1B gene. Science 283, 1544-1548.

Fan, C.D., Lum, M.A., Xu, C., Black, J.D., and Wang, X. (2013). Ubiquitin-dependent regulation of phospho-AKT dynamics by the ubiquitin E3 ligase, NEDD4-1, in the insulin-like growth factor-1 response. J Biol Chem 288, 1674-1684.

Farooqi, I.S., and O'Rahilly, S. (2007). Genetic factors in human obesity. Obes Rev 8 Suppl 1, 37-40.

Faust, I.M., Johnson, P.R., Stern, J.S., and Hirsch, J. (1978). Diet-induced adipocyte number increase in adult rats: a new model of obesity. Am J Physiol 235, E279-286.

Feener, E.P., Backer, J.M., King, G.L., Wilden, P.A., Sun, X.J., Kahn, C.R., and White, M.F. (1993). Insulin stimulates serine and tyrosine phosphorylation in the juxtamembrane region of the insulin receptor. J Biol Chem 268, 11256-11264.

Fischer, A.H., Jacobson, K.A., Rose, J., and Zeller, R. (2008). Hematoxylin and eosin staining of tissue and cell sections. CSH Protoc 2008, pdb prot4986.

Fleming, J.W., McClendon, K.S., and Riche, D.M. (2013). New obesity agents: lorcaserin and phentermine/topiramate. Ann Pharmacother 47, 1007-1016.

Fleury, C., Neverova, M., Collins, S., Raimbault, S., Champigny, O., Levi-Meyrueis, C., Bouillaud, F., Seldin, M.F., Surwit, R.S., Ricquier, D., and Warden, C.H. (1997). Uncoupling protein-2: a novel gene linked to obesity and hyperinsulinemia.Nat Genet 15, 269-272.

Fouladkou, F., Lu, C., Jiang, C., Zhou, L., She, Y., Walls, J.R., Kawabe, H., Brose, N., Henkelman, R.M., Huang, A., et al. (2010). The ubiquitin ligase Nedd4-1 is required for heart development and is a suppressor of thrombospondin-1. J Biol Chem 285, 67706780 . 
Fujisaka, S., Usui, I., Bukhari, A., Ikutani, M., Oya, T., Kanatani, Y., Tsuneyama, K., Nagai, Y., Takatsu, K., Urakaze, M., et al. (2009). Regulatory mechanisms for adipose tissue M1 and M2 macrophages in diet-induced obese mice. Diabetes 58, 2574-2582.

Galitzky, J., Carpene, C., Bousquet-Melou, A., Berlan, M., and Lafontan, M. (1995). Differential activation of beta 1-, beta 2- and beta 3-adrenoceptors by catecholamines in white and brown adipocytes. Fundam Clin Pharmacol 9, 324-331.

Gimeno, R.E. (2007). Fatty acid transport proteins. Curr Opin Lipidol 18, 271-276.

Giralt, M., and Villarroya, F. (2013). White, brown, beige/brite: different adipose cells for different functions? Endocrinology 154, 2992-3000.

Goldstein, B.J., Ahmad, F., Ding, W., Li, P.M., and Zhang, W.R. (1998). Regulation of the insulin signalling pathway by cellular protein-tyrosine phosphatases. Mol Cell Biochem 182, 91-99.

Goodyear, L.J., Giorgino, F., Sherman, L.A., Carey, J., Smith, R.J., and Dohm, G.L. (1995). Insulin receptor phosphorylation, insulin receptor substrate-1 phosphorylation, and phosphatidylinositol 3-kinase activity are decreased in intact skeletal muscle strips from obese subjects. J Clin Invest 95, 2195-2204.

Guo, H., Qiao, G., Ying, H., Li, Z., Zhao, Y., Liang, Y., Yang, L., Lipkowitz, S., Penninger, J.M., Langdon, W.Y., and Zhang, J. (2012). E3 ubiquitin ligase Cbl-b regulates Pten via Nedd4 in T cells independently of its ubiquitin ligase activity. Cell Rep $1,472-482$.

Han, L., Wang, P., Zhao, G., Wang, H., Wang, M., Chen, J., and Tong, T. (2013a). Upregulation of SIRT1 by 17beta-estradiol depends on ubiquitin-proteasome degradation of PPAR-gamma mediated by NEDD4-1. Protein Cell 4, 310-321.

Han, S.O., Kommaddi, R.P., and Shenoy, S.K. (2013b). Distinct roles for beta-arrestin2 and arrestin-domain-containing proteins in beta2 adrenergic receptor trafficking. EMBO Rep 14, 164-171.

Hauser, S., Adelmant, G., Sarraf, P., Wright, H.M., Mueller, E., and Spiegelman, B.M. (2000). Degradation of the peroxisome proliferator-activated receptor gamma is linked to ligand-dependent activation. J Biol Chem 275, 18527-18533.

Heim, K.E., Morrell, J.S., Ronan, A.M., and Tagliaferro, A.R. (2002). Effects of ketamine-xylazine and isoflurane on insulin sensitivity in dehydroepiandrosterone sulfate-treated minipigs (Sus scrofa domestica). Comp Med 52, 233-237.

Hellmer, J., Marcus, C., Sonnenfeld, T., and Arner, P. (1992). Mechanisms for differences in lipolysis between human subcutaneous and omental fat cells. J Clin Endocrinol Metab 75, 15-20. 
Hill, J.O., and Peters, J.C. (1998). Environmental contributions to the obesity epidemic. Science 280, 1371-1374.

Hill, J.O., Wyatt, H.R., and Peters, J.C. (2012). Energy balance and obesity. Circulation 126, 126-132.

Hill, J.O., Wyatt, H.R., Reed, G.W., and Peters, J.C. (2003).Obesity and the environment: where do we go from here? Science 299, 853-855.

Himsworth, H.P. (1940). Insulin Deficiency and Insulin Inefficiency. Br Med J 1, 719722.

Hirasaka, K., Kohno, S., Goto, J., Furochi, H., Mawatari, K., Harada, N., Hosaka, T., Nakaya, Y., Ishidoh, K., Obata, T., et al. (2007). Deficiency of Cbl-b gene enhances infiltration and activation of macrophages in adipose tissue and causes peripheral insulin resistance in mice. Diabetes 56, 2511-2522.

Hoppe, T., Matuschewski, K., Rape, M., Schlenker, S., Ulrich, H.D., and Jentsch, S. (2000). Activation of a membrane-bound transcription factor by regulated ubiquitin/proteasome-dependent processing. Cell 102, 577-586.

Hubbard, S.R. (1997). Crystal structure of the activated insulin receptor tyrosine kinase in complex with peptide substrate and ATP analog. EMBO J 16, 5572-5581.

Ibrahim, M.M. (2010). Subcutaneous and visceral adipose tissue: structural and functional differences. Obes Rev 11, 11-18.

Ingham, R.J., Gish, G., and Pawson, T. (2004). The Nedd4 family of E3 ubiquitin ligases: functional diversity within a common modular architecture. Oncogene 23, 1972-1984.

Jackson, R.S., Creemers, J.W., Ohagi, S., Raffin-Sanson, M.L., Sanders, L., Montague, C.T., Hutton, J.C., and O'Rahilly, S. (1997). Obesity and impaired prohormone processing associated with mutations in the human prohormone convertase 1 gene. Nat Genet 16, 303-306.

Jaworski, K., Ahmadian, M., Duncan, R.E., Sarkadi-Nagy, E., Varady, K.A., Hellerstein, M.K., Lee, H.Y., Samuel, V.T., Shulman, G.I., Kim, K.H., et al. (2009). AdPLA ablation increases lipolysis and prevents obesity induced by high-fat feeding or leptin deficiency. Nat Med 15, 159-168.

Jequier, E. (2002). Leptin signaling, adiposity, and energy balance. Ann N Y Acad Sci 967, 379-388.

Kahn, B.B., and Flier, J.S. (2000). Obesity and insulin resistance. J Clin Invest 106, 473481. 
Kajimura, S., Seale, P., and Spiegelman, B.M. (2010). Transcriptional control of brown fat development. Cell Metab 11, 257-262.

Kanda, H., Tateya, S., Tamori, Y., Kotani, K., Hiasa, K., Kitazawa, R., Kitazawa, S., Miyachi, H., Maeda, S., Egashira, K., and Kasuga, M. (2006). MCP-1 contributes to macrophage infiltration into adipose tissue, insulin resistance, and hepatic steatosis in obesity. J Clin Invest 116, 1494-1505.

Karpe, F., Dickmann, J.R., and Frayn, K.N. (2011). Fatty acids, obesity, and insulin resistance: time for a reevaluation. Diabetes 60, 2441-2449.

Kawabe, H., Neeb, A., Dimova, K., Young, S.M., Jr., Takeda, M., Katsurabayashi, S., Mitkovski, M., Malakhova, O.A., Zhang, D.E., Umikawa, M., et al. (2010). Regulation of Rap2A by the ubiquitin ligase Nedd4-1 controls neurite development. Neuron 65, 358372.

Kennedy, G.E. (1992). Quality in grandparent/grandchild relationships. Int J Aging Hum Dev 35, 83-98.

Kim, J.Y., van de Wall, E., Laplante, M., Azzara, A., Trujillo, M.E., Hofmann, S.M., Schraw, T., Durand, J.L., Li, H., Li, G., et al. (2007). Obesity-associated improvements in metabolic profile through expansion of adipose tissue. J Clin Invest 117, 2621-2637.

Kim, Y.B., Nikoulina, S.E., Ciaraldi, T.P., Henry, R.R., and Kahn, B.B. (1999). Normal insulin-dependent activation of Akt/protein kinase B, with diminished activation of phosphoinositide 3-kinase, in muscle in type 2 diabetes. J Clin Invest 104, 733-741.

Kitamura, T., Kitamura, Y., Kuroda, S., Hino, Y., Ando, M., Kotani, K., Konishi, H., Matsuzaki, H., Kikkawa, U., Ogawa, W., and Kasuga, M. (1999). Insulin-induced phosphorylation and activation of cyclic nucleotide phosphodiesterase $3 \mathrm{~B}$ by the serinethreonine kinase Akt. Mol Cell Biol 19, 6286-6296.

Klaman, L.D., Boss, O., Peroni, O.D., Kim, J.K., Martino, J.L., Zabolotny, J.M., Moghal, N., Lubkin, M., Kim, Y.B., Sharpe, A.H., et al. (2000). Increased energy expenditure, decreased adiposity, and tissue-specific insulin sensitivity in protein-tyrosine phosphatase 1B-deficient mice. Mol Cell Biol 20, 5479-5489.

Koncarevic, A., Jackman, R.W., and Kandarian, S.C. (2007). The ubiquitin-protein ligase Nedd4 targets Notch1 in skeletal muscle and distinguishes the subset of atrophies caused by reduced muscle tension. FASEB J 21, 427-437.

Kotani, K., Ogawa, W., Matsumoto, M., Kitamura, T., Sakaue, H., Hino, Y., Miyake, K., Sano, W., Akimoto, K., Ohno, S., and Kasuga, M. (1998). Requirement of atypical protein kinase clambda for insulin stimulation of glucose uptake but not for Akt activation in 3T3-L1 adipocytes. Mol Cell Biol 18, 6971-6982. 
Krude, H., Biebermann, H., Luck, W., Horn, R., Brabant, G., and Gruters, A. (1998). Severe early-onset obesity, adrenal insufficiency and red hair pigmentation caused by POMC mutations in humans. Nat Genet 19, 155-157.

Kubota, N., Terauchi, Y., Miki, H., Tamemoto, H., Yamauchi, T., Komeda, K., Satoh, S., Nakano, R., Ishii, C., Sugiyama, T., et al. (1999). PPAR gamma mediates high-fat dietinduced adipocyte hypertrophy and insulin resistance. Mol Cell 4, 597-609.

Kumar, S., Harvey, K.F., Kinoshita, M., Copeland, N.G., Noda, M., and Jenkins, N.A. (1997). cDNA cloning, expression analysis, and mapping of the mouse Nedd4 gene. Genomics 40, 435-443.

Kwak, Y.D., Wang, B., Li, J.J., Wang, R., Deng, Q., Diao, S., Chen, Y., Xu, R., Masliah, E., Xu, H., et al. (2012). Upregulation of the E3 ligase NEDD4-1 by oxidative stress degrades IGF-1 receptor protein in neurodegeneration. J Neurosci 32, 10971-10981.

Kwak, Y.D., Wang, B., Pan, W., Xu, H., Jiang, X., and Liao, F.F. (2010). Functional interaction of phosphatase and tensin homologue (PTEN) with the E3 ligase NEDD4-1 during neuronal response to zinc. J Biol Chem 285, 9847-9857.

Lalonde, R. (2002). The neurobiological basis of spontaneous alternation. Neurosci Biobehav Rev 26, 91-104.

Langin, D., Dicker, A., Tavernier, G., Hoffstedt, J., Mairal, A., Ryden, M., Arner, E., Sicard, A., Jenkins, C.M., Viguerie, N., et al. (2005). Adipocyte lipases and defect of lipolysis in human obesity. Diabetes $54,3190-3197$.

Laustsen, P.G., Michael, M.D., Crute, B.E., Cohen, S.E., Ueki, K., Kulkarni, R.N.,Keller, S.R., Lienhard, G.E., and Kahn, C.R. (2002). Lipoatrophic diabetes in Irs1(-/-)/Irs3(-/-) double knockout mice. Genes Dev 16, 3213-3222.

Laviola, L., Giorgino, F., Chow, J.C., Baquero, J.A., Hansen, H., Ooi, J., Zhu, J., Riedel, H., and Smith, R.J. (1997). The adapter protein Grb10 associates preferentially with the insulin receptor as compared with the IGF-I receptor in mouse fibroblasts. J Clin Invest 99, 830-837.

Lee, C.S., Yi, J.S., Jung, S.Y., Kim, B.W., Lee, N.R., Choo, H.J., Jang, S.Y., Han, J.,Chi, S.G., Park, M., et al. (2010). TRIM72 negatively regulates myogenesis via targeting insulin receptor substrate-1. Cell Death Differ 17, 1254-1265.

Lee, J., and Pilch, P.F. (1994). The insulin receptor: structure, function, and signaling. Am J Physiol 266, C319-334.

Lee, K., Villena, J.A., Moon, Y.S., Kim, K.H., Lee, S., Kang, C., and Sul, H.S. (2003). Inhibition of adipogenesis and development of glucose intolerance by soluble preadipocyte factor-1 (Pref-1). J Clin Invest 111, 453-461. 
Lehr, S., Kotzka, J., Herkner, A., Sikmann, A., Meyer, H.E., Krone, W., and MullerWieland, D. (2000). Identification of major tyrosine phosphorylation sites in the human insulin receptor substrate Gab-1 by insulin receptor kinase in vitro. Biochemistry 39 , 10898-10907.

Lehrke, M., and Lazar, M.A. (2005). The many faces of PPARgamma.Cell 123, 993-999. Li, J.J., Dolios, G., Wang, R., and Liao, F.F. (2014). Soluble beta-amyloid peptides, but not insoluble fibrils, have specific effect on neuronal microRNA expression. PLoS One 9 , e90770.

Li, P., Fan, W., Xu, J., Lu, M., Yamamoto, H., Auwerx, J., Sears, D.D., Talukdar, S., Oh, D., Chen, A., et al. (2011). Adipocyte NCoR knockout decreases PPARgamma phosphorylation and enhances PPARgamma activity and insulin sensitivity. Cell 147, 815-826.

Liang, W., and Fishman, P.H. (2004). Resistance of the human beta1-adrenergic receptor to agonist-induced ubiquitination: a mechanism for impaired receptor degradation. J Biol Chem 279, 46882-46889.

Liao, F.F., and Xu, H. (2009). Insulin signaling in sporadic Alzheimer's disease. Sci Signal 2, pe36.

Lihn, A.S., Pedersen, S.B., and Richelsen, B. (2005). Adiponectin: action, regulation and association to insulin sensitivity. Obes Rev 6, 13-21.

Lin, Q., Wang, J., Childress, C., Sudol, M., Carey, D.J., and Yang, W. (2010). HECT E3 ubiquitin ligase Nedd4-1 ubiquitinates ACK and regulates epidermal growth factor (EGF)-induced degradation of EGF receptor and ACK. Mol Cell Biol 30, 1541-1554.

Lin, S., Thomas, T.C., Storlien, L.H., and Huang, X.F. (2000). Development of high fat diet-induced obesity and leptin resistance in $\mathrm{C} 57 \mathrm{Bl} / 6 \mathrm{~J}$ mice. Int J Obes Relat Metab Disord 24, 639-646.

Liu, F., and Roth, R.A. (1995). Grb-IR: a SH2-domain-containing protein that binds to the insulin receptor and inhibits its function. Proc Natl Acad Sci U S A 92, 10287-10291.

Liu, J.P., Baker, J., Perkins, A.S., Robertson, E.J., and Efstratiadis, A. (1993). Mice carrying null mutations of the genes encoding insulin-like growth factor I (Igf-1) and type 1 IGF receptor (Igflr). Cell 75, 59-72.

Liu, Y., Oppenheim, R.W., Sugiura, Y., and Lin, W. (2009). Abnormal development of the neuromuscular junction in Nedd4-deficient mice. Dev Biol 330, 153-166.

Lowell, B.B., and Bachman, E.S. (2003). Beta-Adrenergic receptors, diet-induced thermogenesis, and obesity. J Biol Chem 278, 29385-29388. 
Lutz, T.A., and Woods, S.C. (2012). Overview of animal models of obesity. Curr Protoc Pharmacol Chapter 5, Unit5 61.

Maeda, K., Cao, H., Kono, K., Gorgun, C.Z., Furuhashi, M., Uysal, K.T., Cao, Q., Atsumi, G., Malone, H., Krishnan, B., et al. (2005). Adipocyte/macrophage fatty acid binding proteins control integrated metabolic responses in obesity and diabetes. Cell Metab 1, 107-119.

Magnifico, A., Ettenberg, S., Yang, C., Mariano, J., Tiwari, S., Fang, S., Lipkowitz, S., and Weissman, A.M. (2003). WW domain HECT E3s target Cbl RING finger E3s for proteasomal degradation. J Biol Chem 278, 43169-43177.

Magre, J., Delepine, M., Khallouf, E., Gedde-Dahl, T., Jr., Van Maldergem, L., Sobel, E., Papp, J., Meier, M., Megarbane, A., Bachy, A., et al. (2001). Identification of the gene altered in Berardinelli-Seip congenital lipodystrophy on chromosome 11q13. Nat Genet $28,365-370$.

Mantovani, A., Sica, A., Sozzani, S., Allavena, P., Vecchi, A., and Locati, M. (2004). The chemokine system in diverse forms of macrophage activation and polarization. Trends Immunol 25, 677-686.

Marino, A., Menghini, R., Fabrizi, M., Casagrande, V., Mavilio, M., Stoehr, R.,Candi, E., Mauriello, A., Moreno-Navarrete, J.M., Gomez-Serrano, M., et al. (2014). ITCH deficiency protects from diet-induced obesity. Diabetes $63,550-561$.

Martinez-Botas, J., Anderson, J.B., Tessier, D., Lapillonne, A., Chang, B.H., Quast, M.J., Gorenstein, D., Chen, K.H., and Chan, L. (2000). Absence of perilipin results in leanness and reverses obesity in Lepr(db/db) mice. Nat Genet 26, 474-479.

McNeel, R.L., and Mersmann, H.J. (1999). Distribution and quantification of beta1-, beta2-, and beta3-adrenergic receptor subtype transcripts in porcine tissues. J Anim Sci 77, 611-621.

Medina-Gomez, G., Gray, S.L., Yetukuri, L., Shimomura, K., Virtue, S., Campbell, M., Curtis, R.K., Jimenez-Linan, M., Blount, M., Yeo, G.S., et al. (2007). PPAR gamma 2 prevents lipotoxicity by controlling adipose tissue expandability and peripheral lipid metabolism. PLoS Genet 3, e64.

Medina-Gomez, G., Virtue, S., Lelliott, C., Boiani, R., Campbell, M.,Christodoulides, C., Perrin, C., Jimenez-Linan, M., Blount, M., Dixon, J., et al. (2005). The link between nutritional status and insulin sensitivity is dependent on the adipocyte-specific peroxisome proliferator-activated receptor-gamma2 isoform. Diabetes 54, 1706-1716.

Mokdad, A.H., Marks, J.S., Stroup, D.F., and Gerberding, J.L. (2004). Actual causes of death in the United States, 2000. JAMA 291, 1238-1245. 
Molero, J.C., Jensen, T.E., Withers, P.C., Couzens, M., Herzog, H., Thien, C.B.,Langdon, W.Y., Walder, K., Murphy, M.A., Bowtell, D.D., et al. (2004). c-Cbl-deficient mice have reduced adiposity, higher energy expenditure, and improved peripheral insulin action. $\mathrm{J}$ Clin Invest 114, 1326-1333.

Molero, J.C., Waring, S.G., Cooper, A., Turner, N., Laybutt, R.,Cooney, G.J., and James, D.E. (2006). Casitas b-lineage lymphoma-deficient mice are protected against high-fat diet-induced obesity and insulin resistance. Diabetes 55, 708-715.

Monami, G., Emiliozzi, V., and Morrione, A. (2008). Grb10/Nedd4-mediated multiubiquitination of the insulin-like growth factor receptor regulates receptor internalization. J Cell Physiol 216, 426-437.

Montague, C.T., Farooqi, I.S., Whitehead, J.P., Soos, M.A., Rau, H., Wareham, N.J., Sewter, C.P., Digby, J.E., Mohammed, S.N., Hurst, J.A., et al. (1997). Congenital leptin deficiency is associated with severe early-onset obesity in humans. Nature 387, 903-908.

Morris, R. (1984). Developments of a water-maze procedure for studying spatial learning in the rat. J Neurosci Methods 11, 47-60.

Motomura, W., Inoue, M., Ohtake, T., Takahashi, N., Nagamine, M., Tanno, S., Kohgo, Y., and Okumura, T. (2006). Up-regulation of ADRP in fatty liver in human and liver steatosis in mice fed with high fat diet. Biochem Biophys Res Commun 340, 1111-1118.

Mueller, E., Drori, S., Aiyer, A., Yie, J., Sarraf, P., Chen, H., Hauser, S.,Rosen, E.D., Ge, K., Roeder, R.G., and Spiegelman, B.M. (2002). Genetic analysis of adipogenesis through peroxisome proliferator-activated receptor gamma isoforms. J Biol Chem 277, 41925-41930.

Muller, U. (1999). Ten years of gene targeting: targeted mouse mutants, from vector design to phenotype analysis. Mech Dev 82, 3-21.

Murdaca, J., Treins, C., Monthouel-Kartmann, M.N., Pontier-Bres, R., Kumar, S., Van Obberghen, E., and Giorgetti-Peraldi, S. (2004). Grb10 prevents Nedd4-mediated vascular endothelial growth factor receptor-2 degradation. J Biol Chem 279, 2675426761.

Nabhan, J.F., Pan, H., and Lu, Q. (2010). Arrestin domain-containing protein 3 recruits the NEDD4 E3 ligase to mediate ubiquitination of the beta2-adrenergic receptor. EMBO Rep 11, 605-611.

Nielsen, S., Guo, Z., Johnson, C.M., Hensrud, D.D., and Jensen, M.D. (2004). Splanchnic lipolysis in human obesity. J Clin Invest 113, 1582-1588. 
Ntambi, J.M., Miyazaki, M., Stoehr, J.P., Lan, H., Kendziorski, C.M., Yandell, B.S., Song, Y., Cohen, P., Friedman, J.M., and Attie, A.D. (2002). Loss of stearoyl-CoA desaturase-1 function protects mice against adiposity. Proc Natl Acad Sci U S A 99, 11482-11486.

Ogden, C.L., Carroll, M.D., Kit, B.K., and Flegal, K.M. (2012). Prevalence of obesity and trends in body mass index among US children and adolescents, 1999-2010. JAMA $307,483-490$.

Pajvani, U.B., Trujillo, M.E., Combs, T.P., Iyengar, P., Jelicks, L., Roth, K.A., Kitsis, R.N., and Scherer, P.E. (2005). Fat apoptosis through targeted activation of caspase 8: a new mouse model of inducible and reversible lipoatrophy. Nature medicine 11, 797-803.

Patwari, P., Emilsson, V., Schadt, E.E., Chutkow, W.A., Lee, S., Marsili, A., Zhang, Y., Dobrin, R., Cohen, D.E., Larsen, P.R., et al. (2011). The arrestin domain-containing 3 protein regulates body mass and energy expenditure. Cell Metab 14, 671-683.

Persaud, A., Alberts, P., Hayes, M., Guettler, S., Clarke, I., Sicheri, F., Dirks, P., Ciruna, B., and Rotin, D. (2011). Nedd4-1 binds and ubiquitylates activated FGFR1 to control its endocytosis and function. EMBO J 30, 3259-3273.

Petersen, K.F., and Shulman, G.I. (2006). Etiology of insulin resistance. Am J Med 119, S10-16.

Phillips, R.G., and LeDoux, J.E. (1992). Differential contribution of amygdala and hippocampus to cued and contextual fear conditioning. Behav Neurosci 106, 274-285.

Qatanani, M., and Lazar, M.A. (2007). Mechanisms of obesity-associated insulin resistance: many choices on the menu. Genes Dev 21, 1443-1455.

Qiao, L., Kinney, B., Schaack, J., and Shao, J. (2011). Adiponectin inhibits lipolysis in mouse adipocytes. Diabetes 60, 1519-1527.

Rabinowitz, D., and Zierler, K.L. (1961). Forearm metabolism in obesity and its response to intra-arterial insulin. Evidence for adaptive hyperinsulinism. Lancet 2, 690-692.

Ramos, F.J., Langlais, P.R., Hu, D., Dong, L.Q., and Liu, F. (2006). Grb10 mediates insulin-stimulated degradation of the insulin receptor: a mechanism of negative regulation. Am J Physiol Endocrinol Metab 290, E1262-1266.

Reger, M.A., Watson, G.S., Green, P.S., Wilkinson, C.W., Baker, L.D., Cholerton, B., Fishel, M.A., Plymate, S.R., Breitner, J.C., DeGroodt, W., et al. (2008). Intranasal insulin improves cognition and modulates beta-amyloid in early AD. Neurology 70, 440-448. 
Rising, R., Harper, I.T., Fontvielle, A.M., Ferraro, R.T., Spraul, M., and Ravussin, E. (1994). Determinants of total daily energy expenditure: variability in physical activity. Am J Clin Nutr 59, 800-804.

Rondinone, C.M., Carvalho, E., Rahn, T., Manganiello, V.C., Degerman, E., and Smith, U.P. (2000). Phosphorylation of PDE3B by phosphatidylinositol 3-kinase associated with the insulin receptor. J Biol Chem 275, 10093-10098.

Rondinone, C.M., Wang, L.M., Lonnroth, P., Wesslau, C., Pierce, J.H., and Smith, U. (1997). Insulin receptor substrate (IRS) 1 is reduced and IRS-2 is the main docking protein for phosphatidylinositol 3-kinase in adipocytes from subjects with non-insulindependent diabetes mellitus. Proc Natl Acad Sci U S A 94, 4171-4175.

Rosen, E.D., and MacDougald, O.A. (2006). Adipocyte differentiation from the inside out. Nat Rev Mol Cell Biol 7, 885-896.

Rosen, E.D., Walkey, C.J., Puigserver, P., and Spiegelman, B.M. (2000). Transcriptional regulation of adipogenesis. Genes Dev 14, 1293-1307.

Rotin, D., and Kumar, S. (2009). Physiological functions of the HECT family of ubiquitin ligases. Nat Rev Mol Cell Biol 10, 398-409.

Rotin, D., Staub, O., and Haguenauer-Tsapis, R. (2000). Ubiquitination and endocytosis of plasma membrane proteins: role of Nedd4/Rsp5p family of ubiquitin-protein ligases. $\mathrm{J}$ Membr Biol 176, 1-17.

Rougier, J.S., Albesa, M., Abriel, H., and Viard, P. (2011). Neuronal precursor cellexpressed developmentally down-regulated 4-1 (NEDD4-1) controls the sorting of newly synthesized $\mathrm{Ca}(\mathrm{V}) 1.2$ calcium channels. J Biol Chem 286, 8829-8838.

Saha, P.K., Kojima, H., Martinez-Botas, J., Sunehag, A.L., and Chan, L. (2004). Metabolic adaptations in the absence of perilipin: increased beta-oxidation and decreased hepatic glucose production associated with peripheral insulin resistance but normal glucose tolerance in perilipin-null mice. J Biol Chem 279, 35150-35158.

Sakamoto, K.M. (2002). Ubiquitin-dependent proteolysis: its role in human diseases and the design of therapeutic strategies. Mol Genet Metab 77, 44-56.

Sarjeant, K., and Stephens, J.M. (2012). Adipogenesis. Cold Spring Harb Perspect Biol 4, $\mathrm{a} 008417$.

Scheffner, M., Nuber, U., and Huibregtse, J.M. (1995). Protein ubiquitination involving an E1-E2-E3 enzyme ubiquitin thioester cascade. Nature 373, 81-83. 
Schwarz, L.A., Hall, B.J., and Patrick, G.N. (2010). Activity-dependent ubiquitination of GluA1 mediates a distinct AMPA receptor endocytosis and sorting pathway. J Neurosci 30, 16718-16729.

Shenoy, S.K., McDonald, P.H., Kohout, T.A., and Lefkowitz, R.J. (2001). Regulation of receptor fate by ubiquitination of activated beta 2-adrenergic receptor and beta-arrestin. Science 294, 1307-1313.

Shenoy, S.K., Xiao, K., Venkataramanan, V., Snyder, P.M., Freedman, N.J., and Weissman, A.M. (2008). Nedd4 mediates agonist-dependent ubiquitination, lysosomal targeting, and degradation of the beta2-adrenergic receptor. J Biol Chem 283, 2216622176.

Shi, Y., and Burn, P. (2004). Lipid metabolic enzymes: emerging drug targets for the treatment of obesity. Nat Rev Drug Discov 3, 695-710.

Sinha, M.K., Ohannesian, J.P., Heiman, M.L., Kriauciunas, A., Stephens, T.W., Magosin, S., Marco, C., and Caro, J.F. (1996). Nocturnal rise of leptin in lean, obese, and noninsulin-dependent diabetes mellitus subjects. J Clin Invest 97, 1344-1347.

Smith, S.J., Cases, S., Jensen, D.R., Chen, H.C., Sande, E., Tow, B., Sanan, D.A., Raber, J., Eckel, R.H., and Farese, R.V., Jr. (2000). Obesity resistance and multiple mechanisms of triglyceride synthesis in mice lacking Dgat. Nat Genet 25, 87-90.

Song, R., Peng, W., Zhang, Y., Lv, F., Wu, H.K., Guo, J., Cao, Y., Pi, Y., Zhang, X., Jin, L., et al. (2013). Central role of E3 ubiquitin ligase MG53 in insulin resistance and metabolic disorders. Nature 494, 375-379.

Spalding, K.L., Arner, E., Westermark, P.O., Bernard, S., Buchholz, B.A., Bergmann, O., Blomqvist, L., Hoffstedt, J., Naslund, E., Britton, T., et al. (2008). Dynamics of fat cell turnover in humans. Nature 453, 783-787.

Speakman, J.R. (2013). Measuring energy metabolism in the mouse - theoretical, practical, and analytical considerations. Front Physiol 4, 34.

Steen, E., Terry, B.M., Rivera, E.J., Cannon, J.L., Neely, T.R., Tavares, R., Xu, X.J., Wands, J.R., and de la Monte, S.M. (2005). Impaired insulin and insulin-like growth factor expression and signaling mechanisms in Alzheimer's disease--is this type 3 diabetes? J Alzheimers Dis 7, 63-80.

Stephens, J.M. (2012). The fat controller: adipocyte development. PLoS Biol 10, e1001436.

Steppan, C.M., Bailey, S.T., Bhat, S., Brown, E.J., Banerjee, R.R., Wright, C.M., Patel, H.R., Ahima, R.S., and Lazar, M.A. (2001). The hormone resistin links obesity to diabetes. Nature 409, 307-312. 
Stice, E., Presnell, K., Shaw, H., and Rohde, P. (2005). Psychological and behavioral risk factors for obesity onset in adolescent girls: a prospective study. J Consult Clin Psychol $73,195-202$.

Strable, M.S., and Ntambi, J.M. (2010). Genetic control of de novo lipogenesis: role in diet-induced obesity. Crit Rev Biochem Mol Biol 45, 199-214.

Strissel, K.J., Stancheva, Z., Miyoshi, H., Perfield, J.W., 2nd, DeFuria, J., Jick, Z., Greenberg, A.S., and Obin, M.S. (2007). Adipocyte death, adipose tissue remodeling, and obesity complications. Diabetes 56, 2910-2918.

Strobel, A., Issad, T., Camoin, L., Ozata, M., and Strosberg, A.D. (1998). A leptin missense mutation associated with hypogonadism and morbid obesity. Nat Genet 18 , 213-215.

Sun, K., Kusminski, C.M., and Scherer, P.E. (2011). Adipose tissue remodeling and obesity. J Clin Invest 121, 2094-2101.

Sun, Y. (2003). Targeting E3 ubiquitin ligases for cancer therapy. Cancer Biol Ther 2, 623-629.

Taniguchi, C.M., Emanuelli, B., and Kahn, C.R. (2006). Critical nodes in signalling pathways: insights into insulin action. Nat Rev Mol Cell Biol 7, 85-96.

Tansey, J.T., Sztalryd, C., Gruia-Gray, J., Roush, D.L., Zee, J.V., Gavrilova, O., Reitman, M.L., Deng, C.X., Li, C., Kimmel, A.R., and Londos, C. (2001). Perilipin ablation results in a lean mouse with aberrant adipocyte lipolysis, enhanced leptin production, and resistance to diet-induced obesity. Proc Natl Acad Sci U S A 98, 6494-6499.

Tappy, L., Felber, J.P., and Jequier, E. (1991). Energy and substrate metabolism in obesity and postobese state. Diabetes Care 14, 1180-1188.

Tavernier, G., Barbe, P., Galitzky, J., Berlan, M., Caput, D.,Lafontan, M., and Langin, D. (1996). Expression of beta3-adrenoceptors with low lipolytic action in human subcutaneous white adipocytes. J Lipid Res 37, 87-97.

Thien, C.B., and Langdon, W.Y. (2005). c-Cbl and Cbl-b ubiquitin ligases: substrate diversity and the negative regulation of signalling responses. Biochem J 391, 153-166.

Thuillier, P., Baillie, R., Sha, X., and Clarke, S.D. (1998). Cytosolic and nuclear distribution of PPARgamma2 in differentiating 3T3-L1 preadipocytes. J Lipid Res 39, 2329-2338.

Tong, Q., Dalgin, G., Xu, H., Ting, C.N., Leiden, J.M., and Hotamisligil, G.S. (2000). Function of GATA transcription factors in preadipocyte-adipocyte transition. Science 290, 134-138. 
Vaisse, C., Clement, K., Guy-Grand, B., and Froguel, P. (1998). A frameshift mutation in human MC4R is associated with a dominant form of obesity. Nat Genet 20, 113-114.

Vecchione, A., Marchese, A., Henry, P., Rotin, D., and Morrione, A. (2003). The Grb10/Nedd4 complex regulates ligand-induced ubiquitination and stability of the insulin-like growth factor I receptor. Mol Cell Biol 23, 3363-3372.

Villena, J.A., Choi, C.S., Wang, Y., Kim, S., Hwang, Y.J., Kim, Y.B., Cline, G., Shulman, G.I., and Sul, H.S. (2008). Resistance to high-fat diet-induced obesity but exacerbated insulin resistance in mice overexpressing preadipocyte factor-1 (Pref-1): a new model of partial lipodystrophy. Diabetes 57, 3258-3266.

Virtue, S., and Vidal-Puig, A. (2010). Adipose tissue expandability, lipotoxicity and the Metabolic Syndrome--an allostatic perspective. Biochim Biophys Acta 1801, 338-349.

Viswanadha, S., and Londos, C. (2006). Optimized conditions for measuring lipolysis in murine primary adipocytes. J Lipid Res 47, 1859-1864.

Viswanadha, S., and Londos, C. (2008). Determination of lipolysis in isolated primary adipocytes. Methods Mol Biol 456, 299-306.

Wang, G., Yang, J., and Huibregtse, J.M. (1999a). Functional domains of the Rsp5 ubiquitin-protein ligase. Mol Cell Biol 19, 342-352.

Wang, Q., Somwar, R., Bilan, P.J., Liu, Z., Jin, J., Woodgett, J.R., and Klip, A. (1999b). Protein kinase B/Akt participates in GLUT4 translocation by insulin in L6 myoblasts. Mol Cell Biol 19, 4008-4018.

Wang, X., Trotman, L.C., Koppie, T., Alimonti, A., Chen, Z., Gao, Z., Wang, J., Erdjument-Bromage, H., Tempst, P., Cordon-Cardo, C., et al. (2007). NEDD4-1 is a proto-oncogenic ubiquitin ligase for PTEN. Cell 128, 129-139.

Wellen, K.E., and Hotamisligil, G.S. (2003). Obesity-induced inflammatory changes in adipose tissue. J Clin Invest 112, 1785-1788.

White, C.R., and Seymour, R.S. (2003). Mammalian basal metabolic rate is proportional to body mass2/3. Proc Natl Acad Sci U S A 100, 4046-4049.

Wick, M.J., Dong, L.Q., Hu, D., Langlais, P., and Liu, F. (2001). Insulin receptormediated p62dok tyrosine phosphorylation at residues 362 and 398 plays distinct roles for binding GTPase-activating protein and Nck and is essential for inhibiting insulinstimulated activation of Ras and Akt. J Biol Chem 276, 42843-42850.

Wilkie, N., and Davies S. (2012). Ubiquitination, E3 ligases and drug discovery Novel technologies for a challenging pathway. Drug Discovery World 2012 Summer. 
Xiao, Y.F., Wang, B., Wang, X., Du, F., Benzinou, M., and Wang, Y.X. (2013).

Xylazine-induced reduction of tissue sensitivity to insulin leads to acute hyperglycemia in diabetic and normoglycemic monkeys. BMC Anesthesiol 13, 33.

Xu, H., Barnes, G.T., Yang, Q., Tan, G., Yang, D., Chou, C.J., Sole, J., Nichols, A.,Ross, J.S., Tartaglia, L.A., and Chen, H. (2003). Chronic inflammation in fat plays a crucial role in the development of obesity-related insulin resistance. J Clin Invest 112, 18211830.

Xu, X., Sarikas, A., Dias-Santagata, D.C., Dolios, G., Lafontant, P.J., Tsai, S.C.,Zhu, W., Nakajima, H., Nakajima, H.O., Field, L.J., et al. (2008). The CUL7 E3 ubiquitin ligase targets insulin receptor substrate 1 for ubiquitin-dependent degradation. Mol Cell 30, 403-414.

Yang, B., Gay, D.L., MacLeod, M.K., Cao, X., Hala, T., Sweezer, E.M., Kappler, J., Marrack, P., and Oliver, P.M. (2008). Nedd4 augments the adaptive immune response by promoting ubiquitin-mediated degradation of Cbl-b in activated T cells. Nat Immunol 9, 1356-1363.

Yang, B., and Kumar, S. (2010). Nedd4 and Nedd4-2: closely related ubiquitin-protein ligases with distinct physiological functions. Cell Death Differ 17, 68-77.

Yeo, G.S., Farooqi, I.S., Aminian, S., Halsall, D.J., Stanhope, R.G., and O'Rahilly, S. (1998). A frameshift mutation in MC4R associated with dominantly inherited human obesity. Nat Genet 20, 111-112.

Yi, J.S., Park, J.S., Ham, Y.M., Nguyen, N., Lee, N.R., Hong, J., Kim, B.W., Lee, H., Lee, C.S., Jeong, B.C., et al. (2013). MG53-induced IRS-1 ubiquitination negatively regulates skeletal myogenesis and insulin signalling. Nat Commun 4, 2354.

Zeng, T., Wang, Q., Fu, J., Lin, Q., Bi, J., Ding, W., Qiao, Y., Zhang, S., Zhao, W., Lin, H., et al. (2014). Impeded Nedd4-1-mediated Ras degradation underlies Ras-driven tumorigenesis. Cell Rep 7, 871-882.

Zhang, J., Fu, M., Cui, T., Xiong, C., Xu, K., Zhong, W., Xiao, Y., Floyd, D., Liang, J., Li, E., et al. (2004). Selective disruption of PPARgamma 2 impairs the development of adipose tissue and insulin sensitivity. Proc Natl Acad Sci U S A 101, 10703-10708.

Zhao, W., Chen, H., Xu, H., Moore, E., Meiri, N., Quon, M.J., and Alkon, D.L. (1999). Brain insulin receptors and spatial memory. Correlated changes in gene expression, tyrosine phosphorylation, and signaling molecules in the hippocampus of water maze trained rats. J Biol Chem 274, 34893-34902.

Zhao, W.Q., and Alkon, D.L. (2001). Role of insulin and insulin receptor in learning and memory. Mol Cell Endocrinol 177, 125-134. 
Zhao, W.Q., Chen, H., Quon, M.J., and Alkon, D.L. (2004). Insulin and the insulin receptor in experimental models of learning and memory. Eur J Pharmacol 490, 71-81. Zhou, Z., Yon Toh, S., Chen, Z., Guo, K., Ng, C.P., Ponniah, S., Lin, S.C., Hong, W., and Li, P. (2003). Cidea-deficient mice have lean phenotype and are resistant to obesity. Nat Genet 35, 49-56.

Zimmermann, R., Strauss, J.G., Haemmerle, G., Schoiswohl, G., Birner-Gruenberger, R., Riederer, M., Lass, A., Neuberger, G., Eisenhaber, F., Hermetter, A., and Zechner, R. (2004). Fat mobilization in adipose tissue is promoted by adipose triglyceride lipase. Science 306, 1383-1386. 


\section{APPENDIX: SUPPLEMENTAL INFORMATION}

For i.c.v. injection of leptin, anesthetized mouse was placed on a stereotaxic frame paded with a heating pad set at $37^{\circ} \mathrm{C}$. The head of mouse was fixed on a palate suppor by the ear bars. A small incision was made through the midline of the scalp to expose the bregma and lamda. The angle of head was ajusted to ensure that the bregma and lamda were in the same horizonal level. The skull was then punctured at a desired localization with a mini drill. A $10 \mu 1$ Hamilton syringe was loaded with leptin and inserted into the lateral ventricle of the brain for the injection (stereotaxic parameters: $-0.7 \mathrm{~mm}$ anteriorposterior, $1.2 \mathrm{~mm}$ medial-lateral, and $-1.9 \mathrm{~mm}$ dorsal-ventral). A total volume of $2 \mu \mathrm{l}$ of leptin $(5 \mu \mathrm{g} / \mu \mathrm{l})$ was injected slowly throught via syringe in $5 \mathrm{~min}$. The syringe was left untouched for an additional 5 min after the injection, and then slowly pulled away. After $30 \mathrm{~min}$, mouse was euthanized; hypothalamus was isolated and snap-frozen until use. Tissue lysates were subjected to Western-blot analysis (Figure A.1). 


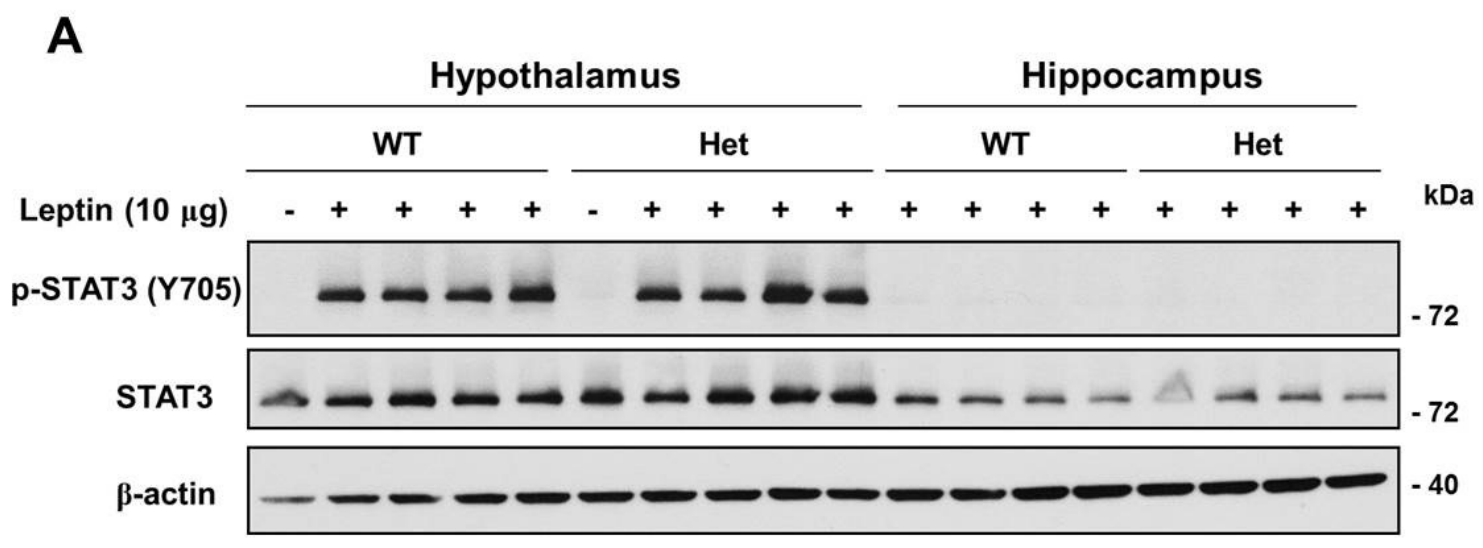

C

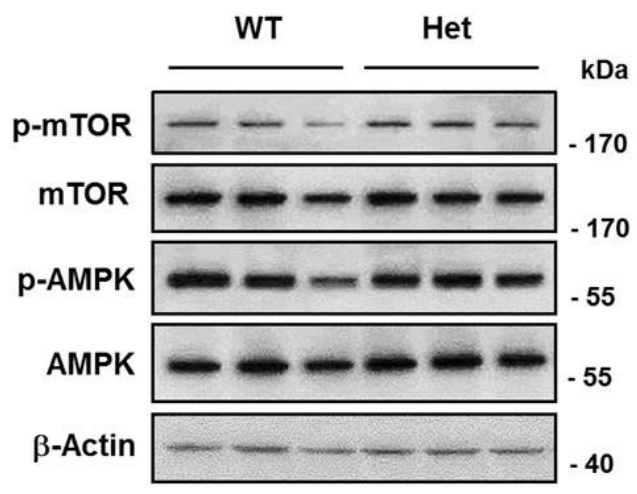

B

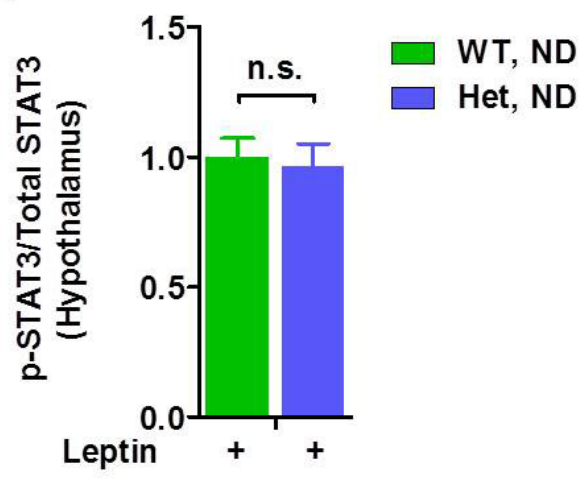

D

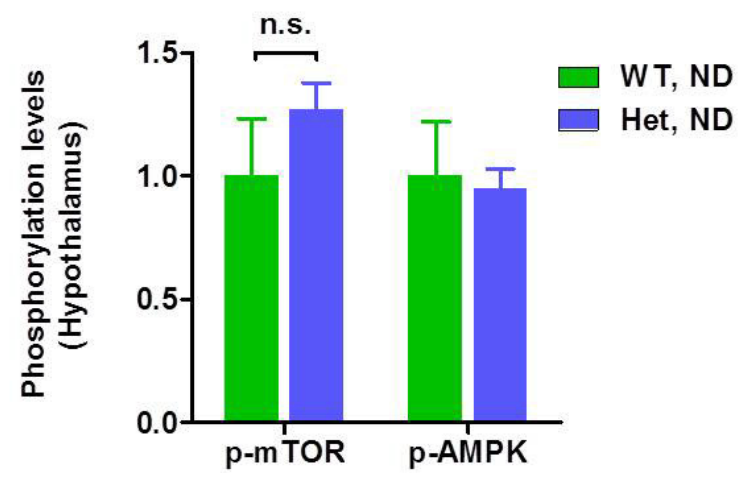

Figure A.1 AMPK, mTOR, and Leptin Signaling in Hypothalamus of Nedd4haploinsuffcient Mice.

(A) Phosphorylation levels of STAT3 by leptin stimulation in the hypothalamus and hippocampus (n=4).(C) Phosphorylation levels of mTOR (Ser2448) and AMPK (Thr172) in hypothalamus in ND-fed mice between 12-16 weeks $(n=3)$. (B) Quantification bar for A. (D) Quantification bar for C. Data represent mean \pm SEM; n.s. no significance. 


\section{VITA}

Jingjing Li was born in Guangxi Province of P.R.China in 1986 and grew up in Guangzhou, Guangdong (P.R.China). During 2004, she attended China Pharmaceutical University in Nanjing, Jiangsu. She attained her bachelor's degree of Pharmaceutical Science in June 2008. In August 2008, she enrolled in the doctoral track of the Integrated Program in Biomedical Sciences at the University of Tennessee Health Science Center. In October 2009, she joined the laboratory of Dr. Francesca-Fang Liao and has been conducted research in aging-related diseases.

Mrs. Li's publications include:

1. Wang, N., Dong, Q., Li, J., Jangra, R.K., Fan, M., Brasier, A.R., Lemon, S.M., Pfeffer, L.M., and Li, K. (2010). Viral induction of the zinc finger antiviral protein is IRF3-dependent but NF-kappaB-independent. J Biol Chem 285, 6080-6090.

2. Kwak, Y.D., Wang, R., Li, J.J., Zhang, Y.W., Xu, H., and Liao, F.F. (2011). Differential regulation of BACE1 expression by oxidative and nitrosative signals. Mol Neurodegener 6, 17.

3. Kwak, Y.D., Wang, B., Li, J.J., Wang, R., Deng, Q., Diao, S., Chen, Y., Xu, R., Masliah, E., Xu, H., et al. (2012). Upregulation of the E3 ligase NEDD4-1 by oxidative stress degrades IGF-1 receptor protein in neurodegeneration.J Neurosci 32, 10971-10981.

4. Wang, R., Li, J.J., Diao, S., Kwak, Y.D., Liu, L., Zhi, L., Bueler, H., Bhat, N.R., Williams, R.W., Park, E.A., and Liao, F.F. (2013). Metabolic stress modulates Alzheimer's beta-secretase gene transcription via SIRT1-PPARgamma-PGC-1 in neurons. Cell Metab 17, 685-694.

5. Chen, Y., Wang, B., Liu, D., Li, J.J., Xue, Y., Sakata, K., Zhu, L.Q.,Heldt, S.A., Xu, H., and Liao, F.F. (2014). Hsp90 chaperone inhibitor 17-AAG attenuates Abetainduced synaptic toxicity and memory impairment. J Neurosci 34, 2464-2470.

6. Li, J.J.*, Dolios, G., Wang, R., and Liao, F.F.* (2014). Soluble beta-amyloid peptides, but not insoluble fibrils, have specific effect on neuronal microRNA expression. PLoS One 9, e90770.

*Corresponding author 